\title{
Global Dynamics of the Hořava-Lifshitz Cosmological Model in a Non-Flat Universe with Non-Zero Cosmological Constant
}

\author{
Fabao Gao ${ }^{1,2, *(D)}$ and Jaume Llibre ${ }^{2}$ (D) \\ 1 School of Mathematical Science, Yangzhou University, Yangzhou 225002, China \\ 2 Departament de Matemàtiques, Universitat Autònoma de Barcelona, Bellaterra, 08193 Barcelona, Spain; \\ jllibre@mat.uab.cat \\ * Correspondence: fbgao@yzu.edu.cn or gaofabao@sina.com
}

check for updates

Citation: Gao, F.; Llibre, J. Global Dynamics of the Hořava-Lifshitz Cosmological Model in a Non-Flat Universe with Non-Zero Cosmological Constant. Universe 2021, 7, 445. https://doi.org/ 10.3390/universe7110445

Academic Editor: Lucas Lombriser

Received: 20 October 2021

Accepted: 15 November 2021

Published: 17 November 2021

Publisher's Note: MDPI stays neutral with regard to jurisdictional claims in published maps and institutional affiliations.

Copyright: (c) 2021 by the authors. Licensee MDPI, Basel, Switzerland. This article is an open access article distributed under the terms and conditions of the Creative Commons Attribution (CC BY) license (https:// creativecommons.org/licenses/by/ $4.0 /)$.
Abstract: When the cosmological constant is non-zero, the dynamics of the cosmological model based on Hořava-Lifshitz gravity in a non-flat universe are characterized by using the qualitative theory of differential equations.

Keywords: global dynamics; Hořava-Lifshitz; non-flat universe; cosmology constant

\section{Introduction}

After the Newtonian era, Einstein put forward the general theory of relativity, and this gave our understanding of gravity, once again, a huge leap forward. In 2009 Hořava proposed a non-relativistic theory of renormalizable gravity [1], which can be reduced to Einstein's general theory of relativity on a large scale. It is named Hořava-Lifshitz gravity together with the scalar field theory of Lifshitz. This theory has inspired many studies and applications in length renormalization [2], entropy argument [3], cosmology [4-30], dark energy [31-35], black holes [36-40], gravitational waves [41] and electromagnetics [42-45]. More information can also be found in the review articles [46-48] and the references therein.

In the past ten years, Leon et al. [9-12] have conducted several excellent studies on the Hořava-Lifshitz cosmological model, whether the curvature $k$ of the universe is zero and whether the cosmological constant $\Lambda$ should be considered. They divided the cosmological model into four types in the Friedmann-Lemaître-Robertson-Walker (FLRW) background spacetime: (1) $\Lambda=0, k=0$; (2) $\Lambda \neq 0, k=0$; (3) $\Lambda=0, k \neq 0$; (4) $\Lambda \neq 0, k \neq 0$. By using the phase-space analysis, they discussed the two-dimensional dynamics of the Hořava-Lifshitz cosmological model under the usual exponential potential, and partially studied its three-dimensional dynamics.

For the important cosmological constant $\Lambda$ that many researchers have been paying attention to, this constant put the Hořava-Lifshitz gravitational theory in a delicate balance, which led to a conflict between its cosmology and observations. Appignani et al. [49] showed that the huge difference between the standard predictions from quantum field theory and the observed value of $\Lambda$ may have a solution in the Hořava-Lifshitz gravity framework. Akarsu et al. [50] investigated the $\Lambda$ in the standard cold dark matter model by introducing graduated dark energy. Their results provided a high probability that the sign of $\Lambda$ could be spontaneously converted, and inferred that the universe had transformed from anti-de Sitter vacua to de Sitter vacua and triggered late acceleration. Carlip [51] proposed that the vacuum fluctuations produce a huge $\Lambda$ and produce a high curvature $k$ on the Planck scale under the standard effective field theory. Although the debate about the shape of the universe has not yet reached an agreement, for the boundaries of the universe are blurred, the odds are 50:1 that the universe is closed if the Planck CMB data are correct [52]. Besides, Valentino et al. [53] also believed that the universe could be a closed three-dimensional sphere, unlike the prediction from the standard $\Lambda$ cold dark matter 
model. The curvature can be positive according to the enhanced lensing amplitude in the cosmic microwave background power spectrum confirmed by the Planck Legacy 2018.

For the case $\Lambda=0$, the global dynamics of the Hořava-Lifshitz scalar field cosmological model under the background of FLRW were described in [14,15], and the case of $\Lambda \neq 0$ with zero curvature has also been addressed in [16]. In the present paper we discuss the global dynamics of a non-flat universe with $\Lambda \neq 0$. We provide detailed information on obtaining cosmological equations in Section 2.

\section{The Cosmological Equations}

In this section, we first briefly recall the classic Hořava-Lifshitz gravitational theory, where the field content can be represented by the space vector $N_{i}$ and scalar $N$, and they are common "lapse" and "shift" variables in general relativity $[1,9,22]$. Then the full metric can be defined as

$$
d s^{2}=-N^{2} d t^{2}+g_{i j}\left(d x^{i}+N^{i} d t\right)\left(d x^{j}+N^{j} d t\right), \quad N_{i}=g_{i j} N^{j},
$$

where $g_{i j}(i, j=1,2,3)$ is a spatial metric. The rescaling conversion meets the conditions $t \rightarrow l^{3} t, x^{i} \rightarrow l x^{i}$, under which $g_{i j}$ and $N$ remain unchanged, but $N^{i}$ is scaled to $N^{i} \rightarrow$ $l^{-2} N_{i}$.

Under the detailed-balance condition, the full gravitational action of Hořava-Lifshitz is written as

$$
\begin{aligned}
S_{g}=\int & d t d^{3} x \sqrt{g} N\left\{\frac{2}{\kappa^{2}}\left(K_{i j} K^{i j}-\lambda K^{2}\right)-\frac{\kappa^{2}}{2 w^{4}} C_{i j} C^{i j}\right. \\
& +\frac{\mu \kappa^{2}}{2 w^{2}} \frac{\epsilon^{i j m}}{\sqrt{g}} R_{i l} \nabla_{j} R_{k}^{l}-\frac{\mu^{2} \kappa^{2}}{8} R_{i j} R^{i j} \\
& \left.-\frac{\mu^{2} \kappa^{2}}{8(3 \lambda-1)}\left(\frac{1-4 \lambda}{4} R^{2}+\Lambda R-3 \Lambda^{2}\right)\right\},
\end{aligned}
$$

where $K_{i j}=\left(\dot{g}_{i j}-\nabla_{i} N_{j}-\nabla_{j} N_{i}\right) /(2 N)$ denotes the extrinsic curvature, $C^{i j}=\epsilon^{i j m} \nabla_{k}$ $\left(4 R_{i}^{j}-R \delta_{i}^{j}\right) /(4 \sqrt{g})$ represents the Cotton tensor and $\epsilon^{i j m} / \sqrt{g}$ is the standard general covariant antisymmetric tensor. The indices are to raise and lower with the metric $g_{i j}$. The parameters $\lambda, \mu$ and $w$ are constants (see [1] for more details).

For the potential $V(\phi)$ we take into account the gravitational action term as follows:

$$
S=\int d t d^{3} x \sqrt{g} N\left(\frac{3 \lambda-1}{4} \frac{\dot{\phi}^{2}}{N^{2}}-V(\phi)\right)
$$

and the metric $N^{i}=0, g_{i j}=a^{2}(t) \gamma_{i j}, \gamma_{i j} d x^{i} d x^{j}=r^{2} d \Omega_{2}^{2}+d r^{2} /\left(1-k r^{2}\right)$. Here the function $a(t)$ is the dimensionless rescaling factor of the expanding universe, and $\gamma_{i j}$ is a constant curvature metric of being maximally symmetric. Without loss of generality, we normalize $\kappa^{2}$ and $N$ to the number one, and then we can describe the cosmological model as

$$
\begin{aligned}
& H^{2}=\frac{\dot{\phi}^{2}}{24}+\frac{V(\phi)}{6(3 \lambda-1)}-\frac{1}{16(3 \lambda-1)^{2}}\left[\frac{\mu^{2} k^{2}}{a^{4}}+\mu^{2} \Lambda^{2}-\frac{2 \mu^{2} \Lambda k}{a^{2}}\right], \\
& \dot{H}+\frac{3}{2} H^{2}=-\frac{\dot{\phi}^{2}}{16}+\frac{V(\phi)}{4(3 \lambda-1)}+\frac{1}{32(3 \lambda-1)^{2}}\left[\frac{\mu^{2} k^{2}}{a^{4}}-3 \mu^{2} \Lambda^{2}-\frac{2 \mu^{2} \Lambda k}{a^{2}}\right], \\
& \ddot{\phi}+3 H \dot{\phi}+\frac{2 V^{\prime}(\phi)}{3 \lambda-1}=0,
\end{aligned}
$$

where $H=\dot{a}(t) / a(t)$ is the Hubble parameter which gives the expansion rate of the universe. 
According to [9-12], we can perform the dimensionless transformation:

$$
x=\frac{\dot{\phi}}{2 \sqrt{6} H}, y=\frac{\sqrt{V(\phi)}}{\sqrt{6} H \sqrt{3 \lambda-1}}, z=\frac{\mu}{4(3 \lambda-1) a^{2} H}, u=\frac{\Lambda \mu}{4(3 \lambda-1) H} .
$$

Then it can be found from Equations (4) and (5) that

$$
x^{2}+y^{2}-(u-k z)^{2}=1, \frac{H^{\prime}}{H}=2 z(z-u)-3 x^{2} .
$$

Therefore, the field equations become the form of the following autonomous dynamical system.

$$
\begin{aligned}
& \frac{d x}{d t}=\sqrt{6} s\left[-x^{2}+(u-z)^{2}+1\right]+x\left[3 x^{2}+2(u-z) z-3\right] \\
& \frac{d z}{d t}=z\left[3 x^{2}+2(u-z) z-2\right] \\
& \frac{d u}{d t}=u\left[3 x^{2}+2(u-z) z\right]
\end{aligned}
$$

where $s=-\frac{1}{\kappa V(\phi)} \frac{d V(\phi)}{d \phi} ; \kappa$ is a constant; $V(\phi)$ is the usual scalar field potential, which admits various mathematical representations (see $[11,13,18,19]$ ) — it can even be presented in a constant form in scalar cosmology $([20,21])$; and $s$ is assumed to be a constant under the usual exponential potentials. For more details on system (7), see Equation (5.72) of [10], or Equations (287)-(290) of [11], or Equations (59)-(61) of [12].

In this paper, we study the global dynamics of system (7) in the physical region of interest

$$
G=\left\{(x, z, u): x^{2}-(u-k z)^{2} \leq 1, u, z \in \mathbb{R}\right\},
$$

where $k=1,0,-1$ corresponding to a closed, flat and open universe, respectively. For the case $k=1$, we note that $f_{+}(x, z, u)=x^{2}-(u-z)^{2}-1=0$ is an invariant surface because there is a polynomial $\mathcal{P}=-2 \sqrt{6} s x+6 x^{2}+4(u-z) z$ such that

$$
\frac{\partial f_{+}}{\partial x} \cdot \frac{d x}{d t}+\frac{\partial f_{+}}{\partial z} \cdot \frac{d z}{d t}+\frac{\partial f_{+}}{\partial u} \cdot \frac{d u}{d t}=\mathcal{P} f_{+} .
$$

The invariant surface here is essential for understanding the complex dynamic behavior of the model (7), because if a point on an orbit of system (7) is located on the invariant surface, then the whole orbit is contained in the surface. However, $f_{-}(x, z, u)=$ $x^{2}-(u+z)^{2}-1=0$ is not an invariant surface for the case $k=-1$. In addition, it is also noted that system (7) is invariant under the three symmetries $(x, z, u) \mapsto(x,-z,-u)$ and $(x, z, u) \mapsto(-x,-z,-u),(x, z, u) \mapsto(-x, z, u)$ if $s=0$; i.e. it is symmetric with respect to the $x$-axis, and additionally with respect to the origin and the plane $x=0$ when $s=0$. Therefore, we divide the study of system (7) into four cases, taking into account the existence or not of the symmetric plane and of the invariant surface.

- $\quad$ Case I: $s \neq 0$ and $k=1$, so system (7) is symmetric with respect to the $x$-axis and it has the invariant surface $f_{+}(x, z, u)=0$.

- $\quad$ Case II: $s \neq 0$ and $k=-1$, so system (7) is symmetric with respect to the $x$-axis and it has not the invariant surface.

- $\quad$ Case III: $s=0$ and $k=1$, so system (7) is symmetric with respect to the origin and with respect to the $x$-axis, and it has the invariant surface $f_{+}(x, z, u)=0$.

- Case IV: $s=0$ and $k=-1$, so system (7) is symmetric with respect to the origin and with respect to the $x$-axis, and it has not the invariant surface.

In Section 3.1 we investigate the phase portraits of case I of system (7) on the invariant planes and surface, along with the local phase portraits at the finite and infinite equilibrium 
points. In Section 3.2 we discuss the phase portraits of case I of system (7) inside the Poincaré ball restricted to the region $G$. Based on these sections, considering the symmetry of system (7), we study the global dynamics of system (7), adding its behavior at infinity in Section 3.3. In Section 4 we study case II in the same way as in case I. In Sections 5 and 6 we describe the global dynamics of system (7) in the closed and open universe models, respectively, when the field potential $V(\phi)$ of the system takes the form of a constant, i.e. $s=0$ in cases III and IV. Moreover, we give the final discussion and summary in Section 7.

\section{Case I: $s \neq 0, k=1$}

\subsection{Phase Portraits on the Invariant Planes and Surface}

In order to analyze in detail the local phase portraits at the finite and infinite equilibrium points and the global phase portrait of system (7) in the region $G$ (refer to [9-11] or [12] again), we first study its phase portraits on the invariant planes $z=0$ and $u=0$, and on the invariant surface $x^{2}-(u-z)^{2}=1$, respectively.

\subsubsection{The Invariant Plane $z=0$}

On this plane system (7) becomes

$$
\begin{aligned}
& \frac{d x}{d t}=\sqrt{6} s\left(-x^{2}+u^{2}+1\right)+3 x\left(x^{2}-1\right), \\
& \frac{d u}{d t}=3 u x^{2} .
\end{aligned}
$$

There are three equilibrium points $e_{1}=(1,0), e_{2}=(-1,0)$ and $e_{3}=(2 s / \sqrt{6}, 0)$ of system (8), where $e_{1}$ has eigenvalues 3 and $6-2 \sqrt{6} s ; e_{2}$ has eigenvalues 3 and $6+2 \sqrt{6}$; and $e_{3}$ has eigenvalues $2 s^{2}$ and $2 s^{2}-3$.

Therefore, the equilibrium point $e_{1}$ is a hyperbolic unstable node when $s<\sqrt{6} / 2$, and it is a hyperbolic unstable saddle when $s>\sqrt{6} / 2$. The equilibrium point $e_{2}$ is a hyperbolic unstable node when $s>-\sqrt{6} / 2$, and it is a hyperbolic unstable saddle when $s<-\sqrt{6} / 2$. The equilibrium point $e_{3}$ is a hyperbolic unstable node when $|s|>\sqrt{6} / 2$, and it is a hyperbolic unstable saddle when $|s|<\sqrt{6} / 2$. Moreover, for $s=-\sqrt{6} / 2, e_{2}=e_{3}$ is a semi-hyperbolic saddle-node, by using the semi-hyperbolic singular point theorem (see Theorem 2.19 in [54] for more details). Similarly, for $s=\sqrt{6} / 2, e_{1}=e_{3}$ is also a semi-hyperbolic saddle-node.

Since the types and stability of these three finite equilibrium points vary with the different values of $s$, we summarize them in Table 1 .

Table 1. Equilibrium points for the different values of $s$, where $e_{1}=(1,0), e_{2}=(-1,0)$ and $e_{3}=(2 s / \sqrt{6}, 0)$.

\begin{tabular}{lc}
\hline Values of $s$ & Equilibrium Points \\
\hline$-\infty<s<-\frac{\sqrt{6}}{2}$ & $e_{1}$ and $e_{3}$ are unstable nodes, $e_{2}$ is an unstable saddle \\
$s=-\frac{\sqrt{6}}{2}$ & $e_{1}$ is an unstable node, $e_{2}=e_{3}$ is an unstable saddle-node \\
\hline$-\frac{\sqrt{6}}{2}<s<\frac{\sqrt{6}}{2}$ & $e_{1}$ and $e_{2}$ are unstable nodes, $e_{3}$ is an unstable saddle \\
\hline$s=\frac{\sqrt{6}}{2}$ & $e_{1}=e_{3}$ is an unstable saddle-node, $e_{2}$ is an unstable node \\
\hline$\frac{\sqrt{6}}{2}<s<+\infty$ & $e_{1}$ is an unstable saddle, $e_{2}$ and $e_{3}$ are unstable nodes \\
\hline
\end{tabular}

By using the Poincaré compactification (see Chapter 5 of [54] for more details), it will help us realize how to draw the vector field of system (8) in the local charts $U_{1}$ and $U_{2}$, and 
then we can determine how the orbits come from or go to infinity. On the local chart $U_{1}$ we let $x=1 / V$ and $u=U / V$; then system (8) becomes

$$
\begin{aligned}
& \frac{d U}{d t}=-U V\left[-3 V+\sqrt{6} s\left(U^{2}+V^{2}-1\right)\right], \\
& \frac{d V}{d t}=-V\left[3-3 V^{2}+\sqrt{6} s V\left(U^{2}+V^{2}-1\right)\right] .
\end{aligned}
$$

Then all the points of system (9) at infinity $V=0$ are equilibrium points, we rescale the time $d \tau_{1}=V d t$, so this system is

$$
\begin{aligned}
& \frac{d U}{d \tau_{1}}=3 U V-\sqrt{6} s U\left(U^{2}+V^{2}-1\right), \\
& \frac{d V}{d \tau_{1}}=-3+3 V^{2}-\sqrt{6} s V\left(U^{2}+V^{2}-1\right) .
\end{aligned}
$$

However, this system has no equilibrium points at infinity $V=0$.

Similarly, on the local chart $U_{2}$ we have $x=U / V$ and $u=1 / V$; thus, system (8) reduces to

$$
\begin{aligned}
& \frac{d U}{d t}=V\left[-3 U V+\sqrt{6} s\left(-U^{2}+V^{2}+1\right)\right], \\
& \frac{d V}{d t}=-3 U^{2} V .
\end{aligned}
$$

On the local chart $U_{2}$ we only need to study the origin $e_{0}=(0,0)$ of system (11). Obviously $e_{0}$ is an equilibrium point. Since its linear part is identically zero, we cannot use the usual eigenvalue method to determine the type of the equilibrium point and its local phase portrait, but we note that the straight line $V=0$ of system (11) is full of equilibrium points, and if the common factor $V$ in system (11) is eliminated, there are no other equilibrium points in $V=0$. When $s>0$, on the positive semi-axis of $V, d V / d t=-3 U^{2} V<0$ indicates that $V$ decreases monotonically, and on the negative semi-axis of $V, d V / d t=-3 U^{2} V>0$ means that $V$ increases monotonically. Near the straight line $U=0, d U / d t=\sqrt{6} s V\left(1+V^{2}\right), U$ increases monotonically when $V>0$, and $U$ decreases monotonically when $V<0$. Therefore, the local phase portrait of the equilibrium point $e_{4}$ of system (11) is shown in Figure 1 a when $s>0$. Similarly, the local phase portrait of $e_{4}$ is illustrated in Figure $1 \mathrm{~b}$ when $s<0$.

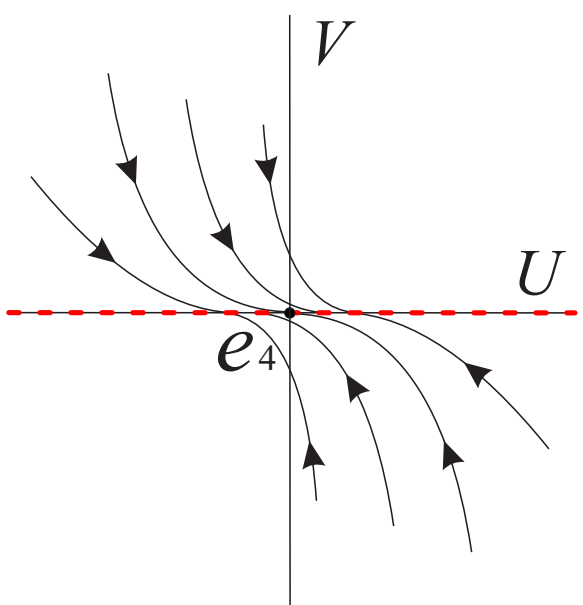

(a)

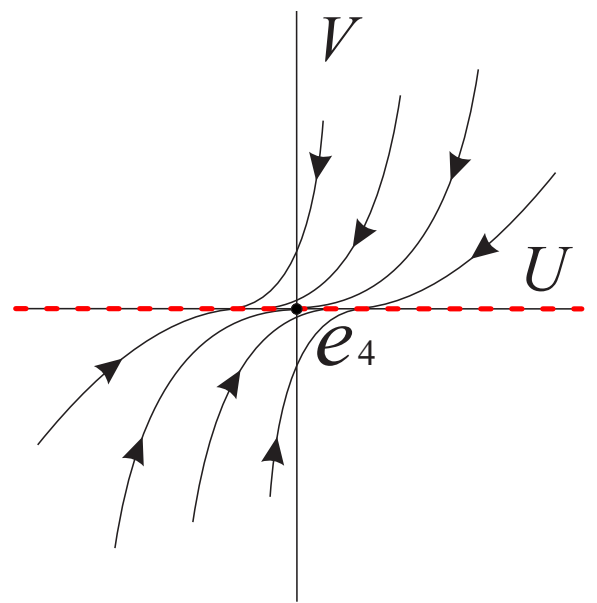

(b)

Figure 1. The local phase portrait at the equilibrium point $e_{o}=(0,0)$ of system (11) in (a) when $s>0$, and in (b) when $s<0$. 
Therefore, the corresponding global phase portrait of system (8) restricted to the region $x^{2}-u^{2} \leq 1$ can be summarized in Figure 2 .

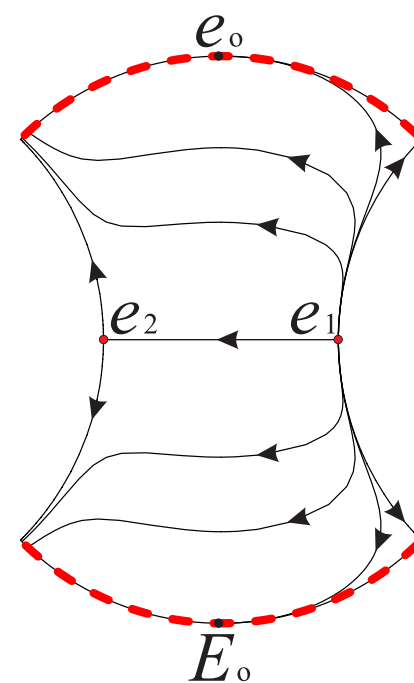

(a)

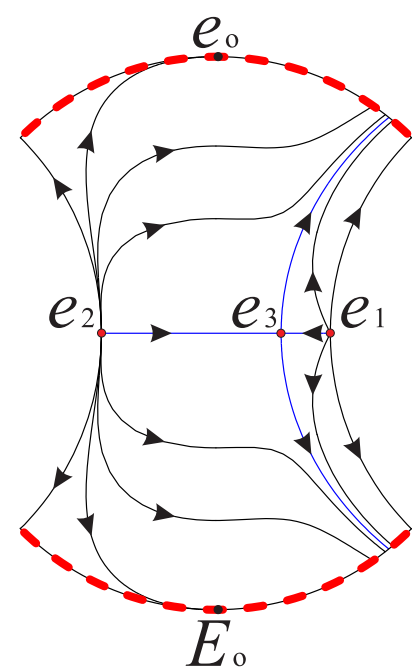

(d)

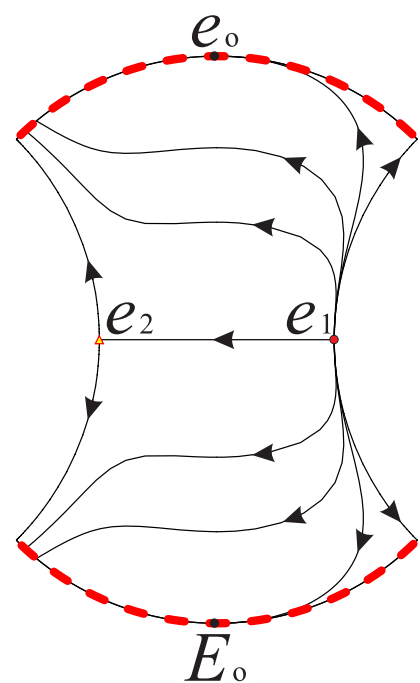

(b)

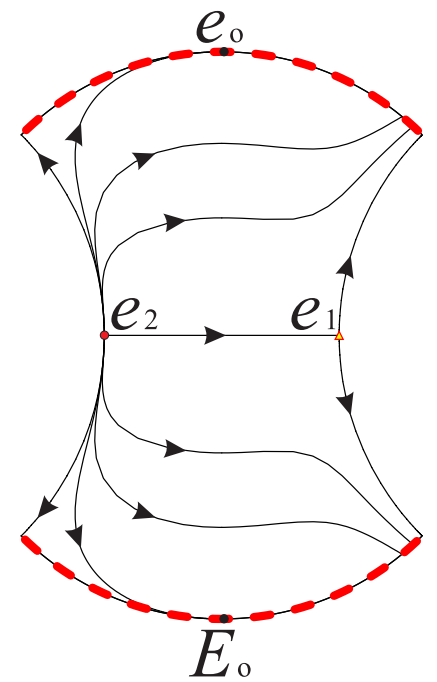

(e)

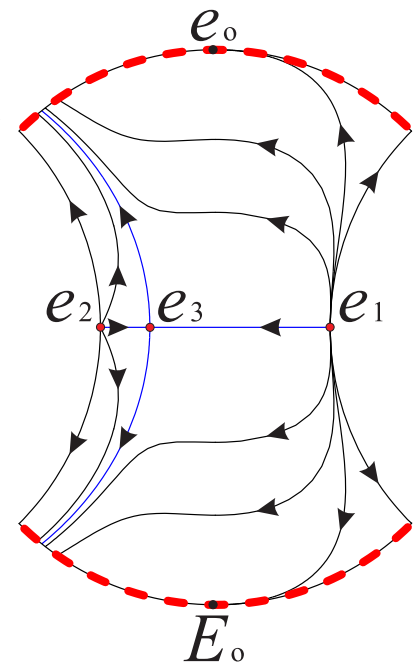

(c)

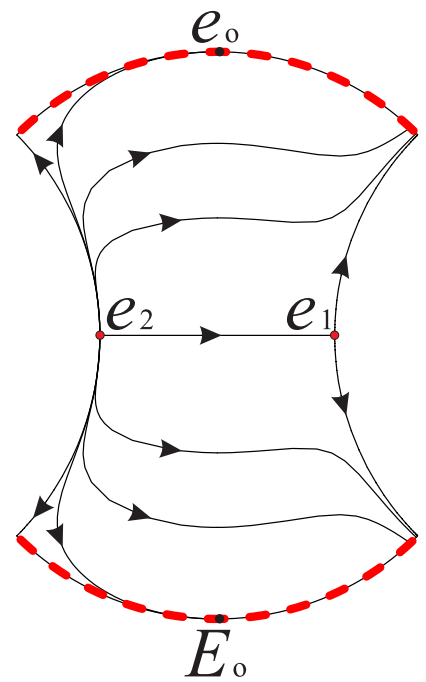

(f)<smiles>[Z]C(C)(C)C</smiles>

Figure 2. The phase portrait on the invariant plane $z=0$ of system (8) restricted to the region $x^{2}-u^{2} \leq 1$ inside the Poincaré disc for different values of $s$ : (a) $s<-\sqrt{6} / 2$, (b) $s=-\sqrt{6} / 2$, (c) $-\sqrt{6} / 2<s<0$, (d) $0<s<\sqrt{6} / 2$, (e) $s=\sqrt{6} / 2$, (f) $s>\sqrt{6} / 2$. Here $E_{o}$ is the diametrically opposite equilibrium point of $e_{0}$ at infinity. 


\subsubsection{The Invariant Plane $u=0$}

On this plane system (7) is

$$
\begin{aligned}
& \frac{d x}{d t}=\sqrt{6} s\left(-x^{2}+z^{2}+1\right)+x\left(3 x^{2}-2 z^{2}-3\right), \\
& \frac{d z}{d t}=z\left(3 x^{2}-2 z^{2}-2\right) .
\end{aligned}
$$

There are five equilibrium points, $e_{1}=(1,0), e_{2}=(-1,0), e_{3}=(2 s / \sqrt{6}, 0), e_{5}=$ $\left(2 /(\sqrt{6} s),-\sqrt{1-s^{2}} / s\right)$ and $e_{6}=\left(2 /(\sqrt{6} s), \sqrt{1-s^{2}} / s\right)$, in system $(12)$ when $s^{2} \neq 1$, and the latter three equilibrium points, $e_{3}, e_{5}$ and $e_{6}$, coincide when $s^{2}=1$. The equilibrium point $e_{1}$ has eigenvalues 1 and $6-2 \sqrt{6} s$, it is a hyperbolic unstable node when $s<\sqrt{6} / 2$, and it is a hyperbolic unstable saddle when $s>\sqrt{6} / 2$. The equilibrium point $e_{2}$ has eigenvalues 1 and $6+2 \sqrt{6} s$, it is a hyperbolic unstable node when $s>-\sqrt{6} / 2$, and it is a hyperbolic unstable saddle when $s<-\sqrt{6} / 2$. The equilibrium point $e_{3}$ has eigenvalues $2 s^{2}-2$ and $2 s^{2}-3$, it is a hyperbolic unstable node when $|s|<1$ or $|s|>\sqrt{6} / 2$, and it is a hyperbolic unstable saddle when $1<|s|<\sqrt{6} / 2$. The equilibrium points $e_{5}$ and $e_{6}$ have the same eigenvalues $-1 / 2-\sqrt{16-15 s^{2}} /(2|s|)$ and $-1 / 2+\sqrt{16-15 s^{2}} /(2|s|)$. They are hyperbolic unstable saddles when $|s|<1$, and they are not real singularities when $|s|>1$.

In addition, for the value $s=-\sqrt{6} / 2$, the equilibrium point $e_{2}=e_{3}$ is a semihyperbolic saddle-node by using the semi-hyperbolic singular point theorem. Similarly, for the value $s=\sqrt{6} / 2$, the equilibrium point $e_{1}=e_{3}$ is also a semi-hyperbolic saddle-node. For the value $s= \pm 1$, the equilibrium point $e_{3}=e_{5}=e_{6}$ is a semi-hyperbolic unstable saddle.

Since the types and stability of these five finite equilibrium points change with different values of $s$, we summarize them in Table 2.

Table 2. Equilibrium points for the different values of $s$, where $e_{1}=(1,0), e_{2}=(-1,0)$, $e_{3}=(2 s / \sqrt{6}, 0), e_{5}=\left(2 /(\sqrt{6} s),-\sqrt{1-s^{2}} / s\right)$ and $e_{6}=\left(2 /(\sqrt{6} s), \sqrt{1-s^{2}} / s\right)$.

\begin{tabular}{ll}
\hline Values of $s$ & \multicolumn{1}{c}{ Equilibrium Points } \\
\hline$-\infty<s<-\frac{\sqrt{6}}{2}$ & $e_{1}$ and $e_{3}$ are unstable nodes, $e_{2}$ is an unstable saddle \\
$s=-\frac{\sqrt{6}}{2}$ & $e_{1}$ is an unstable node, $e_{2}=e_{3}$ is an unstable saddle-node \\
\hline$-\frac{\sqrt{6}}{2}<s<-1$ & $e_{1}$ and $e_{2}$ are unstable nodes, $e_{3}$ is an unstable saddle \\
\hline$s=-1$ & $\begin{array}{l}e_{1} \text { and } e_{2} \text { are unstable nodes, } \\
e_{3}=e_{5}=e_{6} \text { is a semi-hyperbolic unstable saddle }\end{array}$ \\
\hline$-1<s<1$ & $\begin{array}{l}e_{1} \text { and } e_{2} \text { are unstable nodes, } e_{3} \text { is stable node, } \\
e_{5} \text { and } e_{6} \text { are unstable saddles }\end{array}$ \\
\hline$s=1$ & $\begin{array}{l}e_{1} \text { and } e_{2} \text { are unstable nodes, } \\
e_{3}=e_{5}=e_{6} \text { is a semi-hyperbolic unstable saddle }\end{array}$ \\
\hline $1<s<\frac{\sqrt{6}}{2}$ & $e_{1}$ and $e_{2}$ are unstable nodes, $e_{3}$ is an unstable saddle \\
\hline$s=\frac{\sqrt{6}}{2}$ & $e_{1}=e_{3}$ is an unstable saddle-node, $e_{2}$ is an unstable node \\
\hline$\frac{\sqrt{6}}{2}<s<+\infty$ & $e_{1}$ is an unstable saddle, $e_{2}$ and $e_{3}$ are unstable nodes \\
\hline
\end{tabular}

By using the Poincaré compactification again on the local chart $U_{1}$, the system (12) becomes

$$
\begin{aligned}
& \frac{d U}{d t}=-U V\left[-V+\sqrt{6} s\left(U^{2}+V^{2}-1\right)\right] \\
& \frac{d V}{d t}=-V\left[U^{2}(\sqrt{6} s V-2)+(\sqrt{6} s V-3)\left(V^{2}-1\right)\right] .
\end{aligned}
$$


Hence, all the infinite points of system (13) are filled with equilibrium points at $V=0$, doing the time rescaling $d \tau_{2}=V d t$ yields

$$
\begin{aligned}
& \frac{d U}{d \tau_{2}}=U V-\sqrt{6} s U\left(U^{2}+V^{2}-1\right) \\
& \frac{d V}{d \tau_{2}}=-U^{2}(\sqrt{6} s V-2)-(\sqrt{6} s V-3)\left(V^{2}-1\right) .
\end{aligned}
$$

However, this system does not admit any equilibrium point on $V=0$.

Similarly, on the local chart $U_{2}$, system (12) reduces to

$$
\begin{aligned}
\frac{d U}{d t} & =V\left[-U V+\sqrt{6} s\left(-U^{2}+V^{2}+1\right)\right], \\
\frac{d V}{d t} & =V\left[-3 U^{2}+2 V^{2}+2\right] .
\end{aligned}
$$

The origin $e_{4}=(0,0)$ of system (15) is a equilibrium point with eigenvalues 2 and 0 , but it is not semi-hyperbolic because it is not isolated in the set of all equilibrium points. It is noted that the axis $V=0$ is full of equilibrium points of system (15). For the positive semi-axis of $V$ near $e_{4}, d V / d t>0$ means that $V$ increases monotonically, and on the negative semi-axis of $V, d V / d t<0$ indicates that $V$ decreases monotonically. Moreover, $d U / d t=\sqrt{6} s V\left(V^{2}+1\right)$ around the straight line $U=0$, thus $U$ increases monotonically when $s V>0$, and $U$ decreases monotonically when $s V<0$. Therefore, the local phase portrait of the semi-hyperbolic equilibrium point $e_{4}$ in system (15) is illustrated in Figure 3a when $s>0$. Similarly, the local phase portrait of $e_{4}$ is shown in Figure $3 \mathrm{~b}$ for when $s<0$.

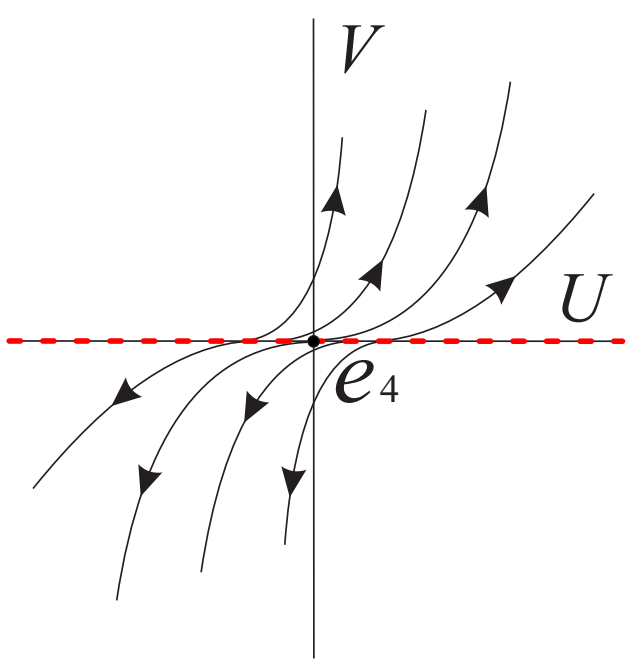

(a)

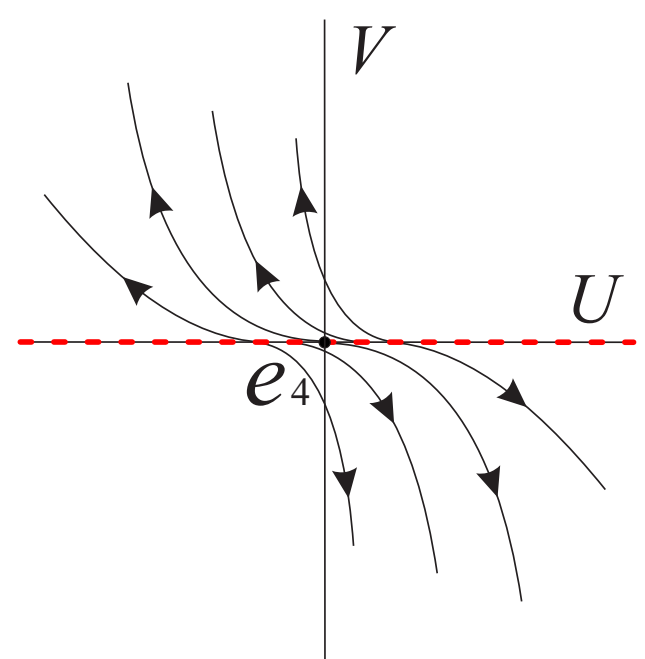

(b)

Figure 3. The local phase portrait at the equilibrium point $e_{4}=(0,0)$ of system (15) in (a) when $s>0$, and in (b) when $s<0$.

Hence, the corresponding global phase portrait of system (12) restricted to the region $x^{2}-z^{2} \leq 1$ is illustrated in Figures 4 and 5. However, we need to pay attention that the global phase portrait of system (12) is similar when $-\sqrt{6} / 2<s<-1$ and $s=-1$ (see Figure $4 \mathrm{c}, \mathrm{d}$ ). The main difference is that the equilibrium point $e_{3}$ is a hyperbolic unstable saddle when $-\sqrt{6} / 2<s<-1$, while $s=-1$ it is a semi-hyperbolic unstable saddle. The same situation occurs when $s=1$ and $1<s<\sqrt{6} / 2$ (see Figure $5 b, c$ ). 


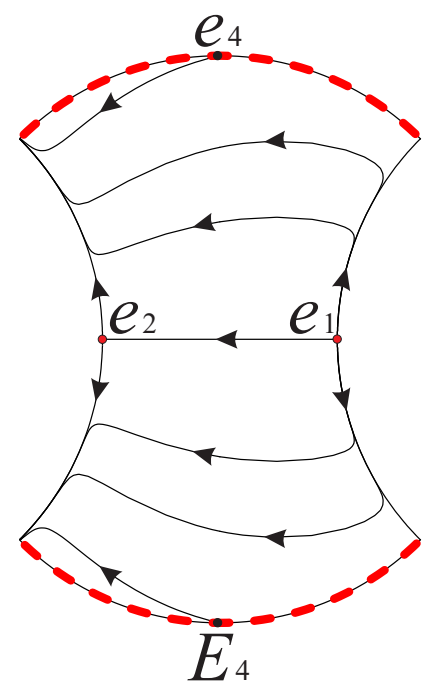

(a)

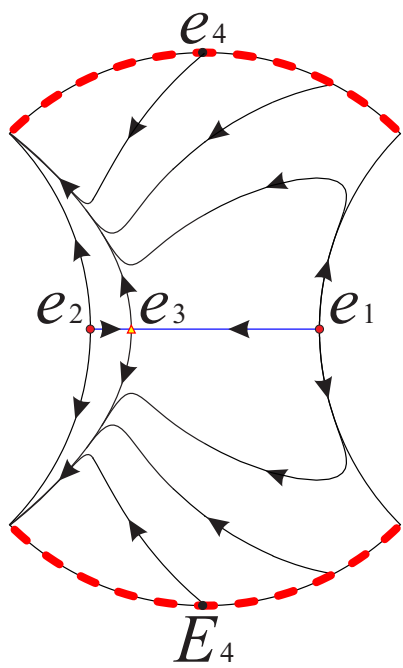

(d)

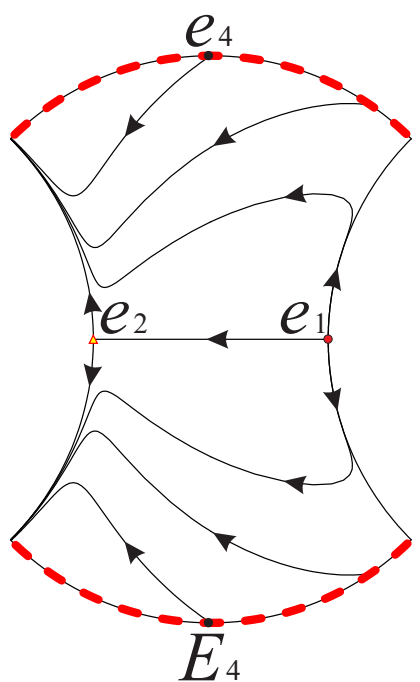

(b)

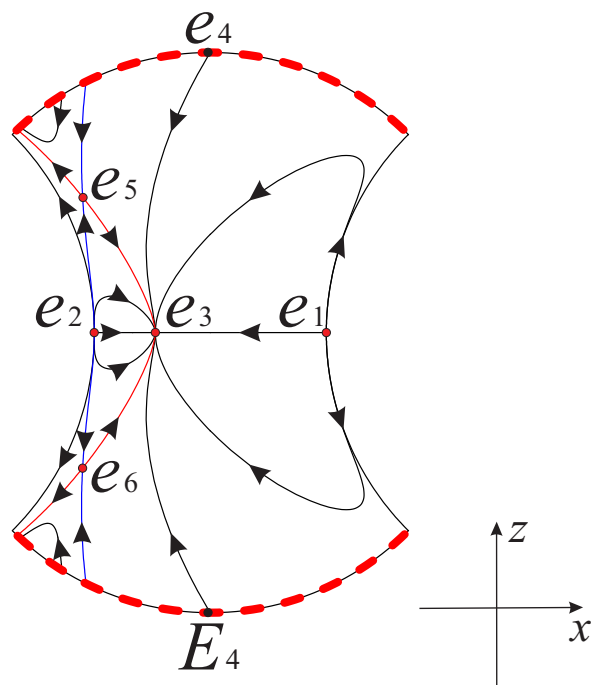

(e)

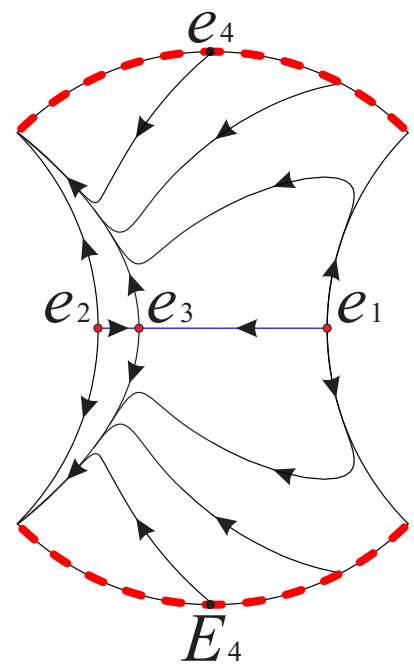

(c)

Figure 4. The phase portrait on the invariant plane $u=0$ restricted to the region $x^{2}-z^{2} \leq 1$ inside the Poincaré disc for different values of $s$ : (a) $s<-\sqrt{6} / 2$, (b) $s=-\sqrt{6} / 2$, (c) $-\sqrt{6} / 2<s<-1$, (d) $s=-1,(\mathbf{e})-1<s<0$. Here $E_{4}$ is the diametrically opposite equilibrium point of $e_{4}$ at infinity. 


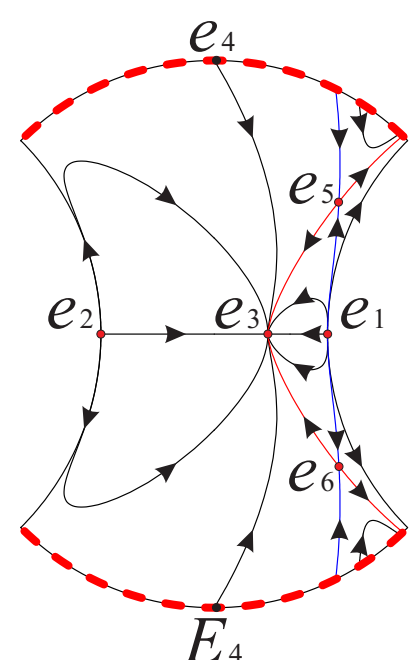

(a)

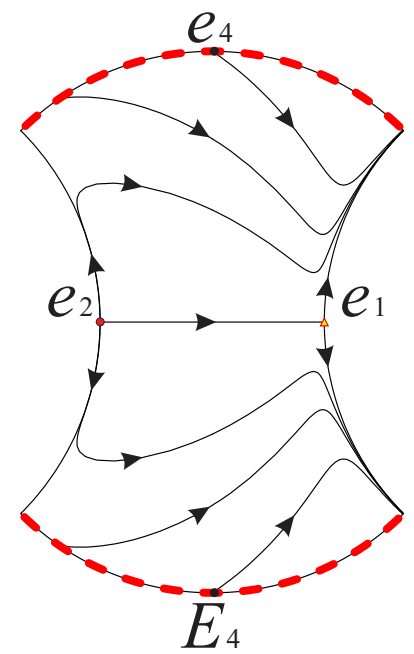

(d)

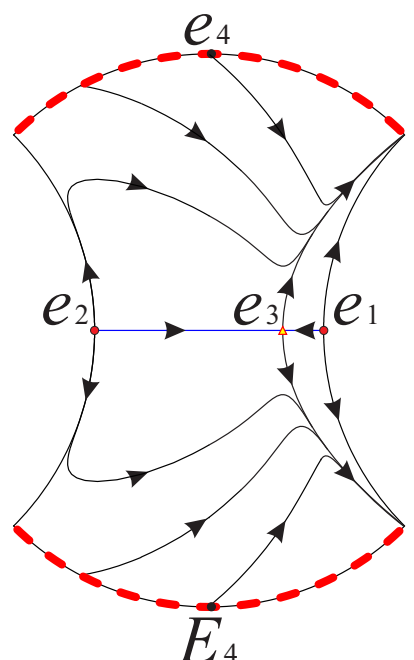

(b)

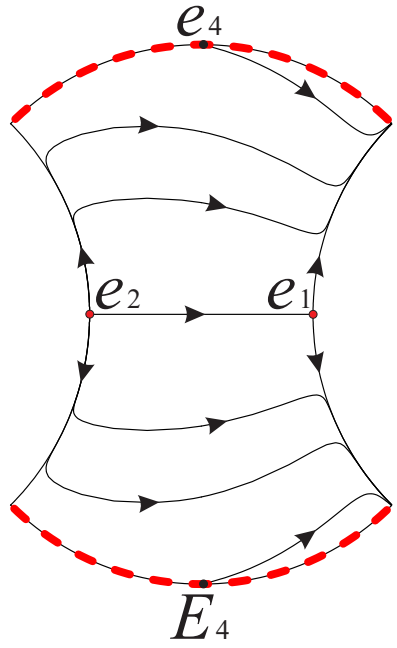

(e)

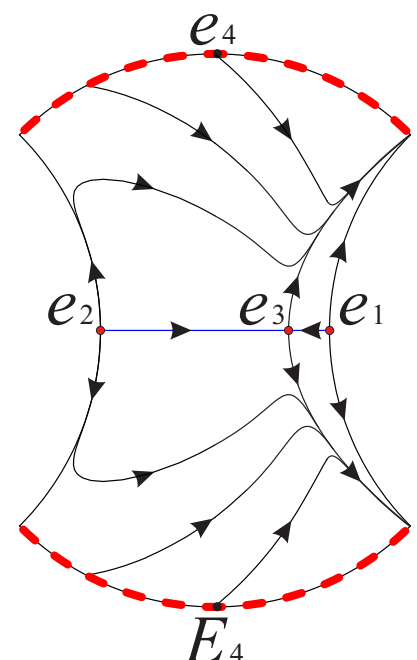

(c)

Figure 5. The phase portrait on the invariant plane $u=0$ restricted to the region $x^{2}-z^{2} \leq 1$ inside the Poincaré disc for different values of $s$ : (a) $0<s<1$, (b) $s=1$, (c) $1<s<\sqrt{6} / 2$, (d) $s=\sqrt{6} / 2$, (e) $s>\sqrt{6} / 2$.

3.1.3. The Invariant Surface $f_{+}(x, z, u)=0$

On this surface system (7) becomes

$$
\begin{aligned}
& \frac{d x}{d t}=x\left(x^{2}-1+2 u \sqrt{x^{2}-1}\right) \\
& \frac{d u}{d t}=u\left(x^{2}+2+2 u \sqrt{x^{2}-1}\right)
\end{aligned}
$$

when $u \geq z$, and for the case $u \leq z$ system (7) reads

$$
\begin{aligned}
& \frac{d x}{d t}=x\left(x^{2}-1-2 u \sqrt{x^{2}-1}\right) \\
& \frac{d u}{d t}=u\left(x^{2}+2-2 u \sqrt{x^{2}-1}\right) .
\end{aligned}
$$


Note that $|x| \geq 1$; thus, for the case $u \geq z$, system (16) admits two equilibrium points $e_{7}=(1,0)$ and $e_{8}=(-1,0)$. In addition we also note that system (16) is symmetric with respect to $u$-axis under the symmetry $(x, u) \mapsto(-x, u)$, so we only need to discuss the equilibrium point $e_{7}$. However, the functions on the right side of system (16) have no derivative at $e_{7}$, which means that the local dynamics near $e_{7}$ cannot be studied by the methods in the previous Sections 3.1.1 and 3.1.2. When $x=1$, the first equation in system (16) is always equal to zero, and the second equation is simplified to $d u / d t=3 u$, i.e. on the invariant straight line $x=1$ the solution is $u(t)=c e^{3 t}$ ( $c$ is an arbitrary constant), which indicates that $u(t)$ tends to infinity in forward time and leads to $e_{7}$ in backward time.

We know the dynamics on $x=1$ near $e_{7}$, but we want to know the dynamics in a neighborhood of $e_{7}$. To this end we set $\sqrt{x^{2}-1}=y>0(|x|>1)$, i.e. $x= \pm \sqrt{1+y^{2}}$ $(y>0)$, considering the aforementioned symmetry. We only discuss the case $x=\sqrt{1+y^{2}}$ $(y>0)$ here, so system (9) can be rewritten as follows:

$$
\begin{aligned}
& \frac{d y}{d t}=(y+2 u)\left(1+y^{2}\right), \\
& \frac{d u}{d t}=u\left(3+2 y u+y^{2}\right) .
\end{aligned}
$$

It is obvious that system (18) has a fictitious equilibrium point $(0,0)$ because $y>0$, and its eigenvalues are 1 and 3 , respectively, i.e. $e_{9}$ is a fictitious hyperbolic unstable node. The first equation of system (18) shows that when $y+2 u>0$, so $y$ increases monotonically. In contrast, if $y+2 u<0$, then $y$ decreases monotonically. Note that $x$ and $y$ have the same monotonicity when $y>0$, so the local phase portrait of system (16) near $e_{7}$ and system $(18)$ near $(0,0)$ have the same local phase portrait. Then considering the symmetry $(y, u) \mapsto(-y,-u)$ of system (18), we can find that the local phase portrait of system (16) is shown in Figure 6.

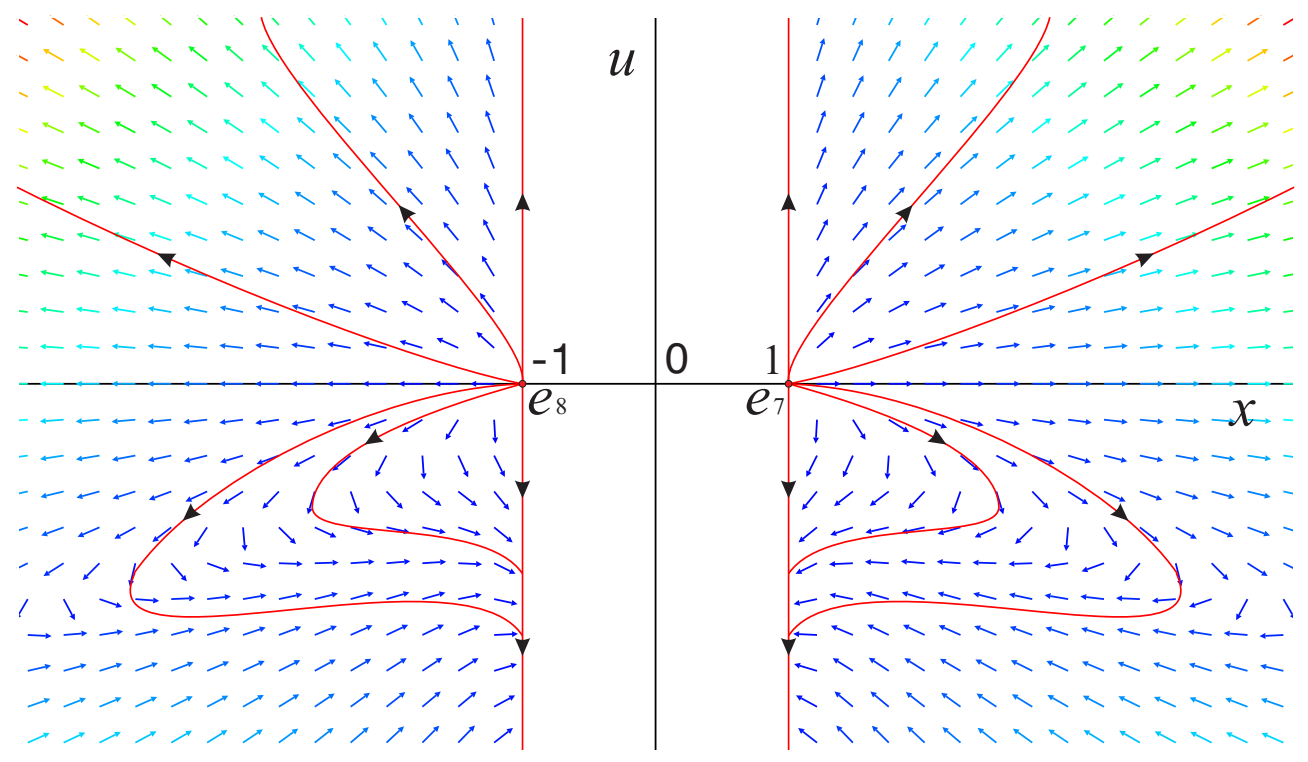

Figure 6. The phase portrait on the invariant surface $f_{+}(x, z, u)=0$ restricted to the region $u \geq z$.

Similarly, the local phase portrait of system (17) is shown in Figure 7. 


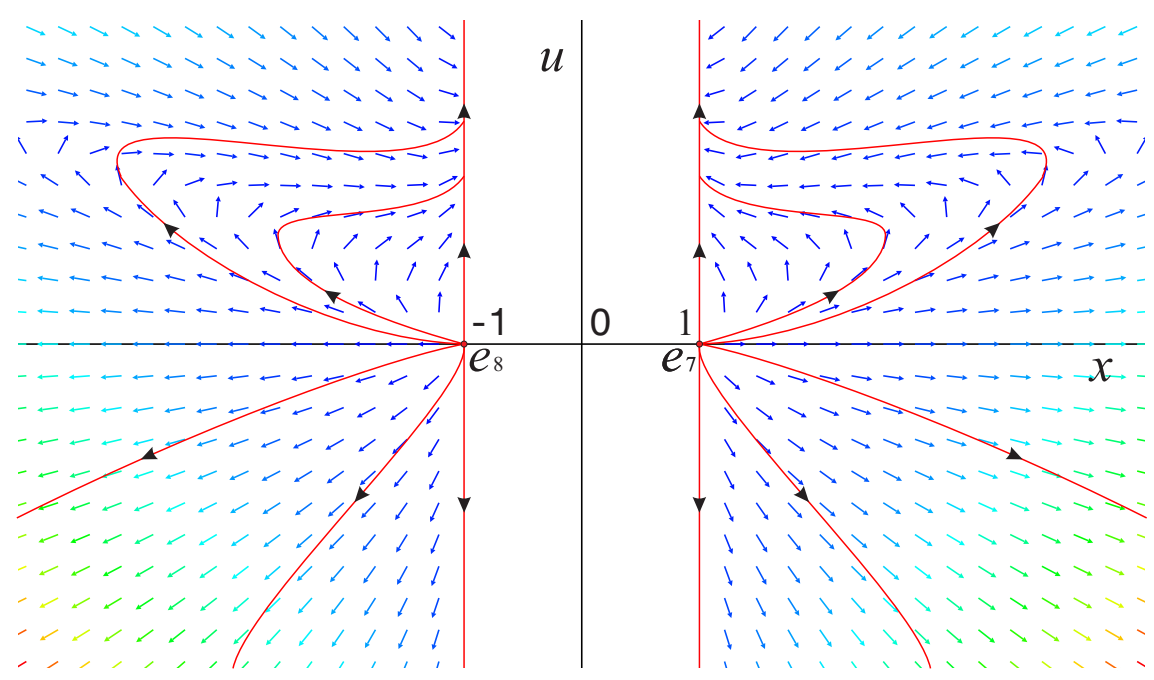

Figure 7. The phase portrait on the invariant surface $f_{+}(x, z, u)=0$ restricted to the region $u \leq z$.

Note that system (16) can be transformed into a polynomial differential system by letting $y=\sqrt{x^{2}-1}$ when $|x| \neq 1$. In order to study the dynamic behavior of the equilibrium points of system (16) at infinity, we first study the infinite equilibrium points of system (18). On the local chart $U_{1}$ system (18) is

$$
\begin{aligned}
& \frac{d U}{d t}=2 U(1-U) V^{2}, \\
& \frac{d V}{d t}=-(1+2 U) V\left(1+V^{2}\right) .
\end{aligned}
$$

It is easy to find that all the infinite points of system (19) at $V=0$ are equilibrium points. Performing time scale transformation $d \tau_{3}=V d t$ to eliminate the common factor $V$ in system (19) gives

$$
\begin{aligned}
& \frac{d U}{d \tau_{3}}=2 U(1-U) V \\
& \frac{d V}{d \tau_{3}}=-(1+2 U)\left(1+V^{2}\right) .
\end{aligned}
$$

This system has a unique equilibrium point in $V=0, e_{9}=(-1 / 2,0)$ with eigenvalues $\pm \sqrt{3}$, it is a hyperbolic saddle.

On the local chart $U_{2}$ system (18) reads

$$
\begin{aligned}
& \frac{d U}{d t}=2(1-U) V^{2} \\
& \frac{d V}{d t}=-U(2+U) V-3 V^{3}
\end{aligned}
$$

Using time rescaling $d \tau_{4}=V d t$ we obtain

$$
\begin{aligned}
& \frac{d U}{d \tau_{4}}=2(1-U) V \\
& \frac{d V}{d \tau_{4}}=-U(2+U)-3 V^{2} .
\end{aligned}
$$

The origin $e_{10}=(0,0)$ is an equilibrium point, which is a hyperbolic stable center with eigenvalue $\pm 3 i$ ( $i$ is the imaginary unit). Hence $e_{10}$ is either a weak focus or a center, but since $\mathcal{H}_{f}=\left(2 U-U^{2}+V^{2}-2 / 3\right) /(U-1)^{3}$ is a first integral of system (22) defined at $(0,0), e_{10}$ is a center. Then the global phase portrait of system (18) with $y>0$ is shown in Figure 8. 

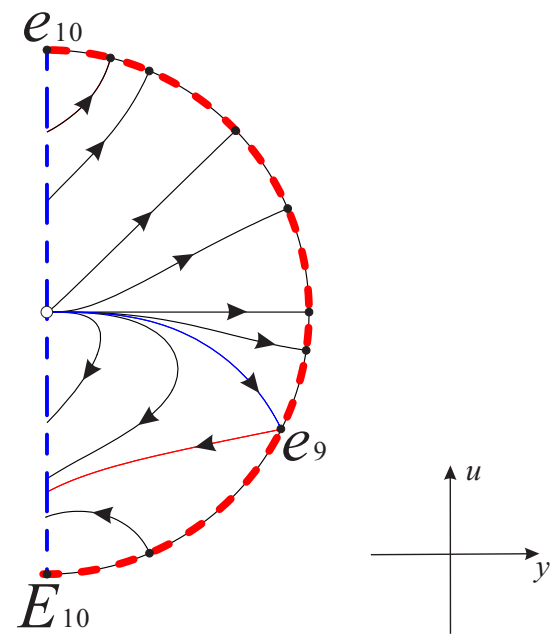

Figure 8. The global phase portrait of system (18) with $y>0$. Here $E_{10}$ is the diametrically opposite equilibrium point of $e_{10}$ at infinity.

Therefore, combining Figure 8 with Figures 6 and 7, we obtain the global phase portrait of systems (16) and (17) as shown in Figures 9 and 10, respectively.
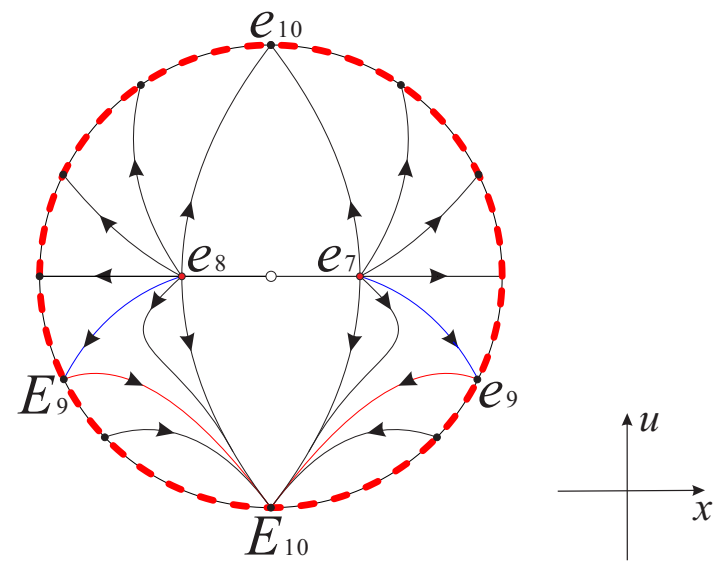

Figure 9. The global phase portrait of system (16) on the invariant surface $f_{+}(x, z, u)=0$ restricted to the region $u \geq z$. Here $E_{9}$ is the symmetric point of $e_{9}$ with respect to the axis $x=0$, and $E_{10}$ is the diametrically opposite equilibrium point of $e_{10}$ at infinity.
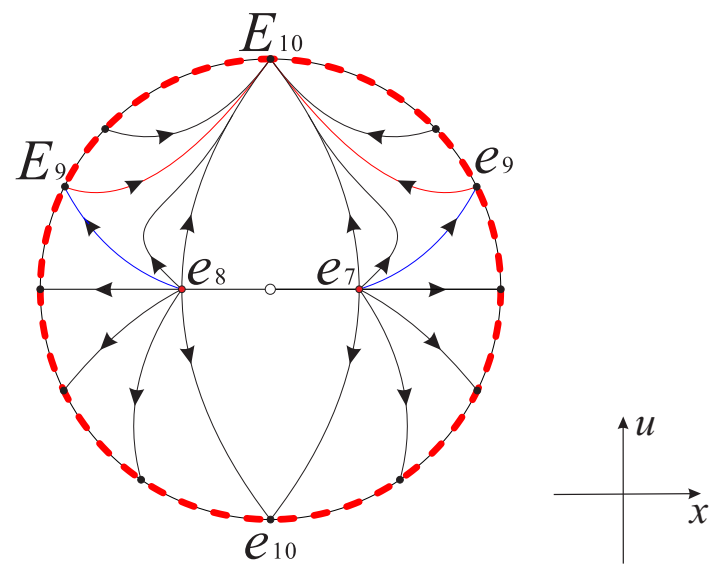

Figure 10. The global phase portrait of system (17) on the invariant surface $f_{+}(x, z, u)=0$ restricted to the region $u \leq z$. 


\subsubsection{The Finite Equilibrium Points}

It is easy to find that there are five finite equilibrium points of system (7) because $s \neq 0$ in case I. The equilibrium point $p_{1}=(1,0,0)$ with eigenvalues 3,1 and $6-2 \sqrt{6} s$, the equilibrium point $p_{2}=(-1,0,0)$ with eigenvalues 3,1 and $6+2 \sqrt{6} s$, the equilibrium points $p_{3}=\left(\sqrt{6} /(3 s),-\sqrt{1-s^{2}} / s, 0\right), p_{4}=\left(\sqrt{6} /(3 s), \sqrt{1-s^{2}} / s, 0\right), p_{3}$ and $p_{4}$ have the same eigenvalues $\left.2,-1 / 2-\sqrt{16-15 s^{2}}\right) /(2|s|)$ and $\left.-1 / 2+\sqrt{16-15 s^{2}}\right) /(2|s|)$, and the equilibrium point $p_{5}=(\sqrt{6} s / 3,0,0)$ with eigenvalues $2 s^{2}, 2 s^{2}-2$, and $2 s^{2}-3$. Since different values of $s$ determine the types of these five equilibrium points, we list the relevant results more clearly in Table 3.

Table 3. Finite equilibrium points for the different values of $s$, where $p_{1}=(1,0,0), p_{2}=(-1,0,0)$, $p_{3}=\left(\sqrt{6} /(3 s),-\sqrt{1-s^{2}} / s, 0\right), p_{4}=\left(\sqrt{6} /(3 s), \sqrt{1-s^{2}} / s, 0\right)$ and $p_{5}=(\sqrt{6} s / 3,0,0)$.

\begin{tabular}{ll}
\hline Values of $s$ & \multicolumn{1}{c}{ Equilibrium Points } \\
\hline$-\infty<s<-\frac{\sqrt{6}}{2}$ & $p_{1}$ and $p_{5}$ are unstable nodes, \\
$p_{2}$ is a saddle
\end{tabular}

\subsubsection{Phase Portrait on the Poincaré Sphere at Infinity}

According to the three-dimensional Poincaré compactification (see [55] for more details), we set $x=1 / z_{3}, z=z_{1} / z_{3}, u=z_{2} / z_{3}$. Thus, on the local chart $U_{1}$, system (7) is rewritten as

$$
\begin{aligned}
\frac{d z_{1}}{d t}= & -z_{1} z_{3}\left[\sqrt{6} s\left(\left(z_{1}-z_{2}\right)^{2}+z_{3}^{2}-1\right)-z_{3}\right], \\
\frac{d z_{2}}{d t}= & -z_{2} z_{3}\left[\sqrt{6} s\left(\left(z_{1}-z_{2}\right)^{2}+z_{3}^{2}-1\right)-3 z_{3}\right], \\
\frac{d z_{3}}{d t}= & z_{3}\left[-3+2 z_{1}^{2}-2 z_{1} z_{2}-\sqrt{6} s\left(\left(z_{1}-z_{2}\right)^{2}-1\right) z_{3}\right. \\
& \left.+3 z_{3}^{2}-\sqrt{6} s z_{3}^{3}\right] .
\end{aligned}
$$

Since the infinity at the different local charts of Poincare sphere corresponds to $z_{3}=0$, then for all $z_{1}, z_{2} \in \mathbb{R}$, system (23) has the equilibrium point $\left(z_{1}, z_{2}, 0\right)$ with eigenvalues $\left\{0,0,2 z_{1}\left(z_{1}-z_{2}\right)-3\right\}$, which means that the local chart $U_{1}$ are full of equilibrium points at infinity. Note that the corresponding eigenvectors are

$$
\begin{gathered}
\{0,1,0\}, \quad\{1,0,0\}, \\
\left\{\frac{\sqrt{6} z_{1} s\left[\left(z_{1}-z_{2}\right)^{2}-1\right]}{3-2 z_{1}\left(z_{1}-z_{2}\right)} \quad \frac{\sqrt{6} z_{2} s\left[\left(z_{1}-z_{2}\right)^{2}-1\right]}{3-2 z_{1}\left(z_{1}-z_{2}\right)}, 1\right\} .
\end{gathered}
$$


By using normally hyperbolic submanifold theorem (see Appendix A for details), the equilibrium point $\left(z_{1}, z_{2}, 0\right)$ has a one-dimensional stable manifold when $2 z_{1}\left(z_{1}-z_{2}\right)<3$ and unstable when $2 z_{1}\left(z_{1}-z_{2}\right)>3$. More details are shown in regions I, II, and III of Figure 11. However, for the equilibrium points on the hyperbola $2 z_{1}\left(z_{1}-z_{2}\right)=3$ there are six local phase portraits in Figure 12. Note that there is one-dimensional stable manifold in region I and one-dimensional unstable manifold in regions II and III filled with infinite equilibrium points. Since the orbits arriving or ending at equilibrium points at infinity in the different regions cannot collide into finite equilibrium points when they tend to equilibrium points on the hyperbola $2 z_{1}\left(z_{1}-z_{2}\right)=3$ coming from both sides of this hyperbola, there is a hyperbolic sector on the equilibrium points of the branches $L_{1}$ and $L_{2}$ of the hyperbola, with the exception of two points $p_{6}$ and $p_{7}$; see the first two pictures in Figure 12 for details.

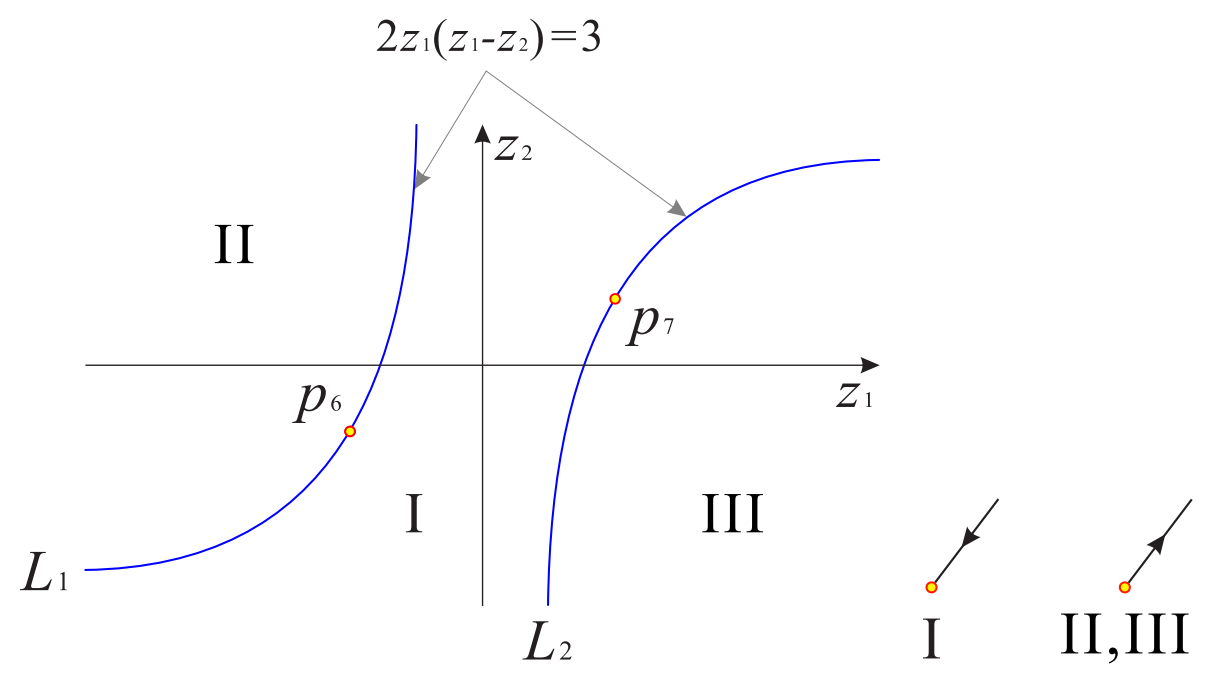

Figure 11. There is an one-dimensional stable manifold in the region I and one-dimensional unstable manifold in regions II and III on the local chart $U_{1}$.
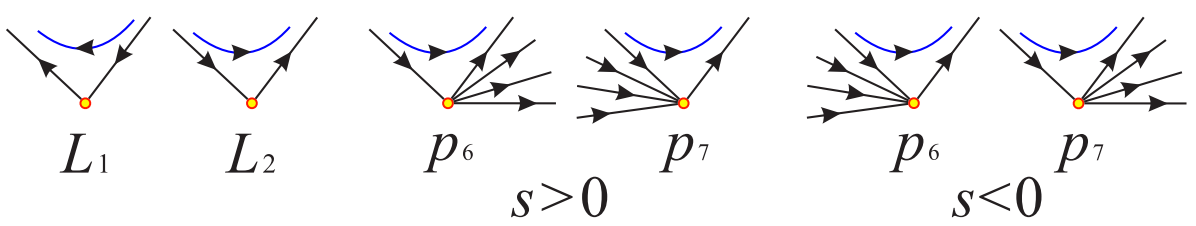

Figure 12. There are six kinds of equilibrium points on the hyperbola $2 z_{1}\left(z_{1}-z_{2}\right)=3$.

Doing time rescaling $d \tau_{5}=z_{3} d t$ system (23) becomes

$$
\begin{aligned}
\frac{d z_{1}}{d \tau_{5}}= & -\sqrt{6} s z_{1}\left[\left(z_{1}-z_{2}\right)^{2}+z_{3}^{2}-1\right]+z_{1} z_{3} \\
\frac{d z_{2}}{d \tau_{5}}= & -\sqrt{6} s z_{2}\left[\left(z_{1}-z_{2}\right)^{2}+z_{3}^{2}-1\right]+3 z_{2} z_{3} \\
\frac{d z_{3}}{d \tau_{5}}= & -3+2 z_{1}^{2}-2 z_{1} z_{2}-\sqrt{6} s z_{3}\left[\left(z_{1}-z_{2}\right)^{2}-1\right] \\
& +3 z_{3}^{2}-\sqrt{6} s z_{3}^{3}
\end{aligned}
$$

At $z_{3}=0$ system (25) has two lines $\left|z_{1}-z_{2}\right|=1$ filled with infinite equilibrium points and these two lines intersect with hyperbola $2 z_{1}\left(z_{1}-z_{2}\right)=3$ at $p_{6}=(-3 / 2,-1 / 2,0)$ and 
$p_{7}=(3 / 2,1 / 2,0)$, respectively. Both of them are hyperbolic with the same eigenvalues $\{-\sqrt{3}, \sqrt{3},-2 \sqrt{6} s\}$ and the corresponding eigenvectors have the following form:

$$
\begin{aligned}
& \left(\frac{\sqrt{3}}{2}, \frac{\sqrt{3}}{2}, 1\right),\left(-\frac{\sqrt{3}}{2},-\frac{\sqrt{3}}{2}, 1\right),\left(\frac{\sqrt{6}\left(8 s^{2}+1\right)}{16 s}, \frac{\sqrt{6}\left(8 s^{2}+5\right)}{48 s}, 1\right) . \\
& \left(-\frac{\sqrt{3}}{2},-\frac{\sqrt{3}}{2}, 1\right),\left(\frac{\sqrt{3}}{2}, \frac{\sqrt{3}}{2}, 1\right),\left(-\frac{\sqrt{6}\left(8 s^{2}+1\right)}{16 s},-\frac{\sqrt{6}\left(8 s^{2}+5\right)}{48 s}, 1\right) .
\end{aligned}
$$

Then $p_{6}$ and $p_{7}$ have an unstable manifold of dimension one (respectively two) and a stable manifold of dimension two (respectively one) if $s>0$ (respectively $s<0$ ); see the last four pictures in Figure 12 for details.

On the local chart $U_{2}$, we let $x=z_{1} / z_{3}, z=1 / z_{3}, u=z_{2} / z_{3}$. Thus, system (7) is

$$
\begin{aligned}
& \frac{d z_{1}}{d t}=z_{3}\left[-z_{1} z_{3}+\sqrt{6} s\left(-z_{1}^{2}+\left(z_{2}-1\right)^{2}+z_{3}^{2}\right)\right], \\
& \frac{d z_{2}}{d t}=2 z_{2} z_{3}^{2} \\
& \frac{d z_{3}}{d t}=z_{3}\left(2-3 z_{1}^{2}-2 z_{2}+2 z_{3}^{2}\right) .
\end{aligned}
$$

By eliminating the common factor $z_{3}$ in system (27) through time scale transformation $d \tau_{6}=z_{3} d t$ we get

$$
\begin{aligned}
& \frac{d z_{1}}{d \tau_{6}}=-z_{1} z_{3}+\sqrt{6} s\left[-z_{1}^{2}+\left(z_{2}-1\right)^{2}+z_{3}^{2}\right], \\
& \frac{d z_{2}}{d \tau_{6}}=2 z_{2} z_{3}, \\
& \frac{d z_{3}}{d \tau_{6}}=2-3 z_{1}^{2}-2 z_{2}+2 z_{3}^{2} .
\end{aligned}
$$

Note that system (28) with $z_{1}=z_{3}=0$ has the unique infinite equilibrium point $p_{8}=(0,1,0)$ with eigenvalues $\{2 \mathrm{i},-2 \mathrm{i}, 0\}$ and eigenvectors $\{0,-i, 1\},\{0, i, 1\},\{1,0,0\}$, so there will be a fold-Hopf bifurcation at the infinite equilibrium point $p_{8}$, sometimes called a zero-pair bifurcation or a Gavrilov-Guckenheimer (see Chapter 5 of [56] for more details). We will not continue to discuss other infinite equilibrium points of this system, because these are already included in the local chart $U_{1}$.

Similarly, on the local chart $U_{3}$, we let $x=z_{1} / z_{3}, z=z_{2} / z_{3}, u=1 / z_{3}$, so system (7) becomes

$$
\begin{aligned}
\frac{d z_{1}}{d t} & =z_{3}\left[-3 z_{1} z_{3}+\sqrt{6} s\left(-z_{1}^{2}+\left(z_{2}-1\right)^{2}+z_{3}^{2}\right)\right], \\
\frac{d z_{2}}{d t} & =-2 z_{2} z_{3}^{2} \\
\frac{d z_{3}}{d t} & =-3 z_{1}^{2} z_{3}+2\left(z_{2}-1\right) z_{2} z_{3} .
\end{aligned}
$$

In this local chart $U_{3}$ we only need to study the infinite equilibria located in its origin because all the other infinite equilibrium points have been studied in the local charts $U_{1}$ and $U_{2}$. After changing the time scale $d \tau_{7}=z_{3} d t$ we obtain 


$$
\begin{aligned}
& \frac{d z_{1}}{d t}=-3 z_{1} z_{3}+\sqrt{6} s\left[-z_{1}^{2}+\left(z_{2}-1\right)^{2}+z_{3}^{2}\right] \\
& \frac{d z_{2}}{d t}=-2 z_{2} z_{3} \\
& \frac{d z_{3}}{d t}=-3 z_{1}^{2}+2\left(z_{2}-1\right) z_{2} .
\end{aligned}
$$

Obviously the origin $(0,0,0)$ is not an equilibrium point of system (30), so we will not continue to investigate other equilibrium points at infinity in the local chart $U_{3}$.

In summary the equilibrium points filling up the infinity with these different stable and unstable manifolds are summarized in Figures 11-13.
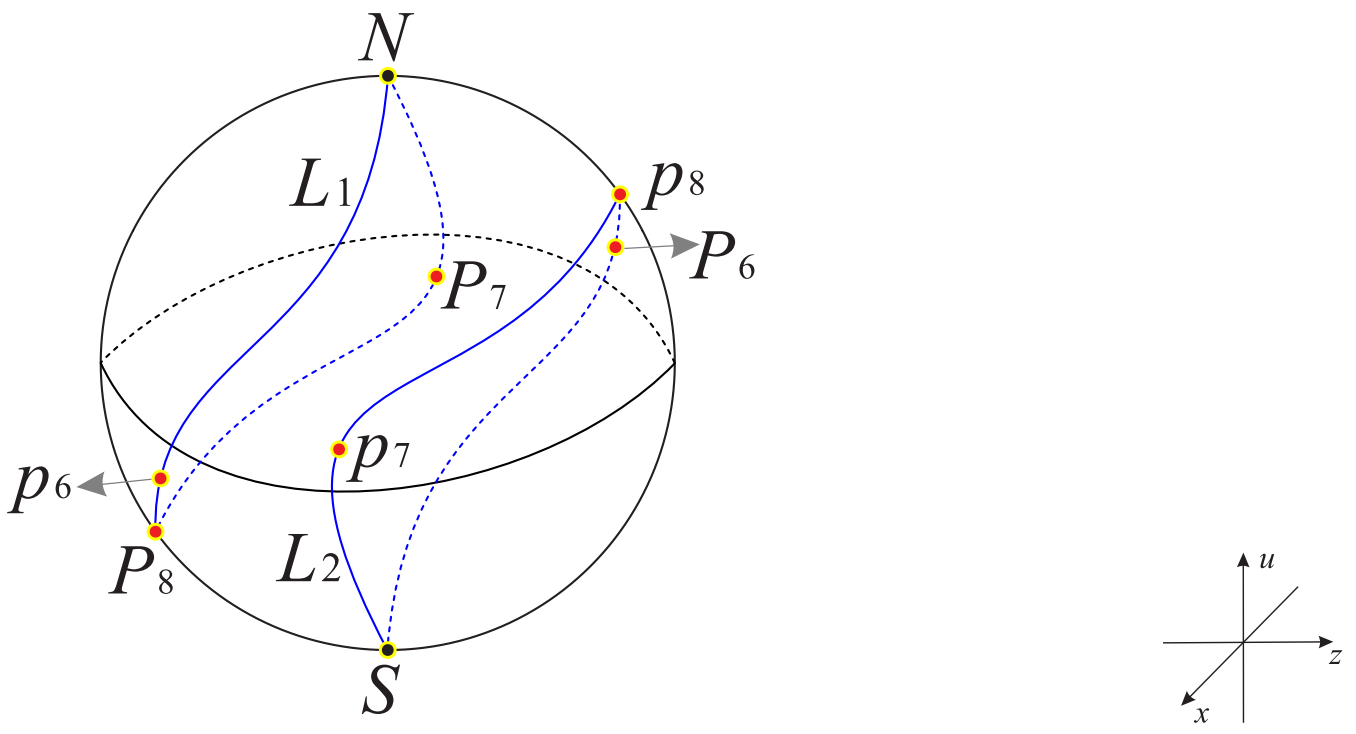

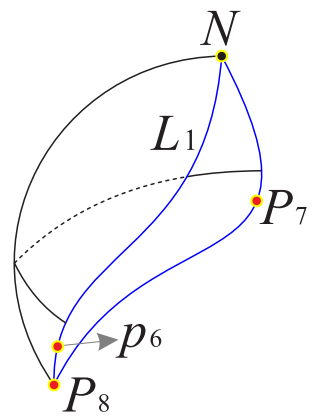

Region II

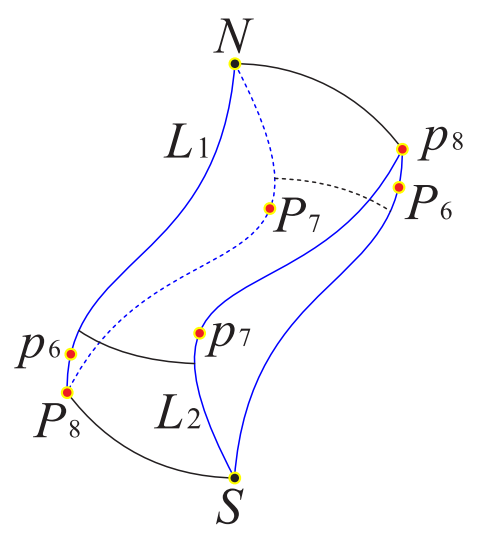

Region I

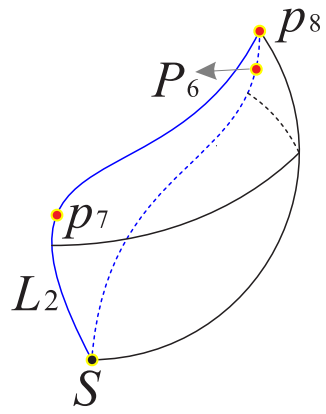

Region III

Figure 13. The sphere $\mathbb{S}^{2}$ (the infinity of $\mathbb{R}^{3}$ ) is filled up with equilibrium points. The stable and unstable manifolds of these equilibrium points are different in regions I, II and III; lines $L_{1}$ and $L_{2}$; and points $p_{6}, p_{7}, p_{8}, P_{6}, P_{7}$ and $P_{8}$ on the sphere defined by the closure of $U_{1}$ and its symmetric closure of $V_{1}$ with respect to the origin of $\mathbb{R}^{3}$. Thus, $P_{6}, P_{7}$ and $P_{8}$ are the symmetric points with respect to the origins of $p_{6}, p_{7}$ and $p_{8}$, respectively. The points $N$ and $S$ denote the north pole and south pole of the Poincaré ball, respectively. 
3.2. Phase Portrait Inside the Poincaré Ball Restricted to the Physical Region of Interest $x^{2}-(u-z)^{2} \leq 1$

Note that system (7) is invariant with respect to the $x$-axis due to the symmetry $(x, z, u) \mapsto(x,-z,-u)$. Now we divide the Poincaré ball restricted to the region $x^{2}-(u-$ $z)^{2}-1 \leq 0$ into four regions as follows:

$$
\begin{aligned}
& R_{1}: z \geq 0, u \geq 0 . \quad R_{2}: z \geq 0, u \leq 0 . \\
& R_{3}: z \leq 0, u \leq 0 . \quad R_{4}: z \leq 0, u \geq 0 .
\end{aligned}
$$

Due to the symmetry with respect to the $x$-axis, we only need to discuss the phase portrait of system (7) in the regions $R_{1}$ and $R_{2}$.

Combining the phase portraits on the invariant surface $f_{+}(x, z, u)=0$, on the invariant planes $z=0$ and $u=0$, and at infinity, we obtain the phase portrait on the boundary of the regions $R_{1}$ and $R_{2}$ as shown in Figures 14-17.

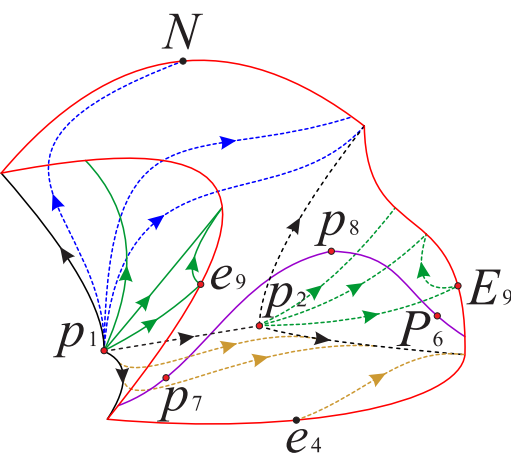

(a)

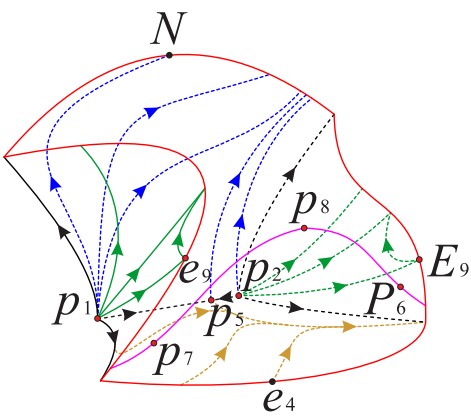

(c)

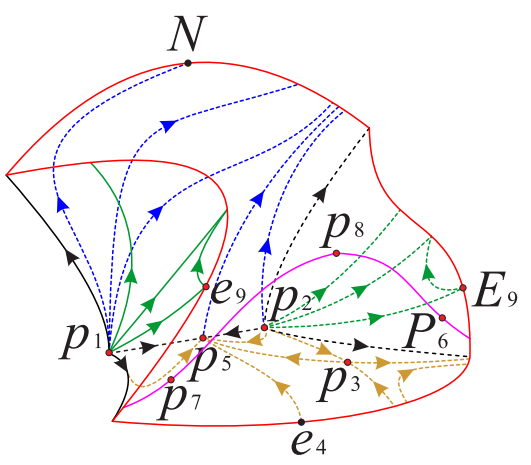

(e)

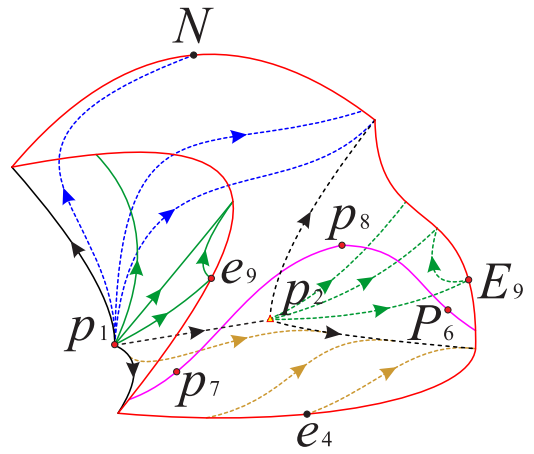

(b)

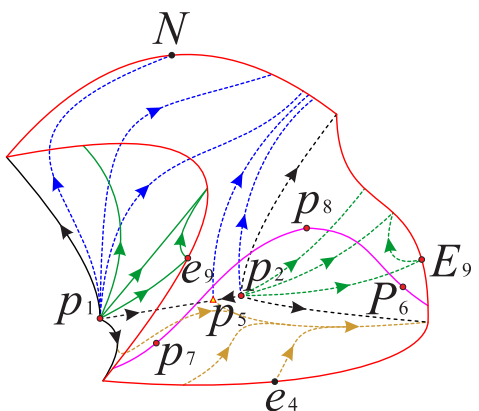

(d)

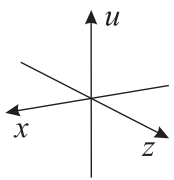

Figure 14. Phase portrait in the boundary of the region $R_{1}$ for different values of $s$ : (a) $s<-\sqrt{6} / 2$, (b) $s=-\sqrt{6} / 2,(\mathbf{c})-\sqrt{6} / 2<s<-1$, (d) $s=-1$, (e) $-1<s<0$. The surfaces above and below the long dashed line represent regions I and III, respectively. 


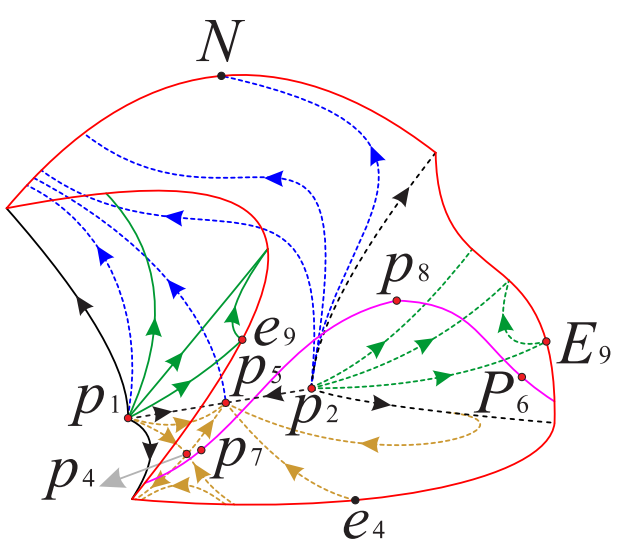

(a)

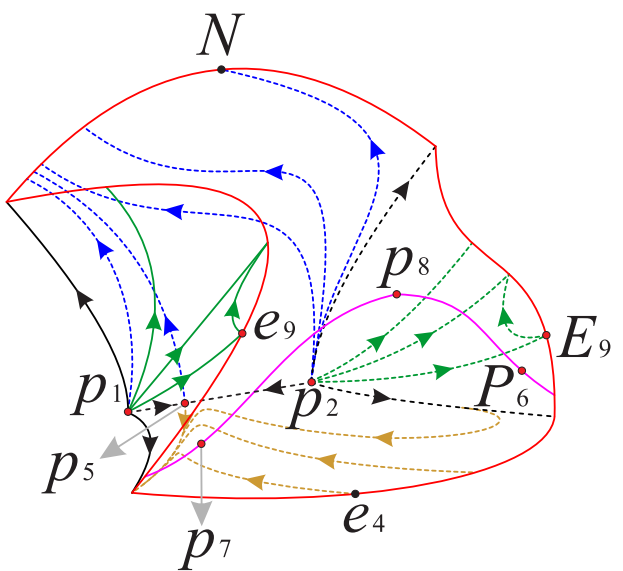

(c)

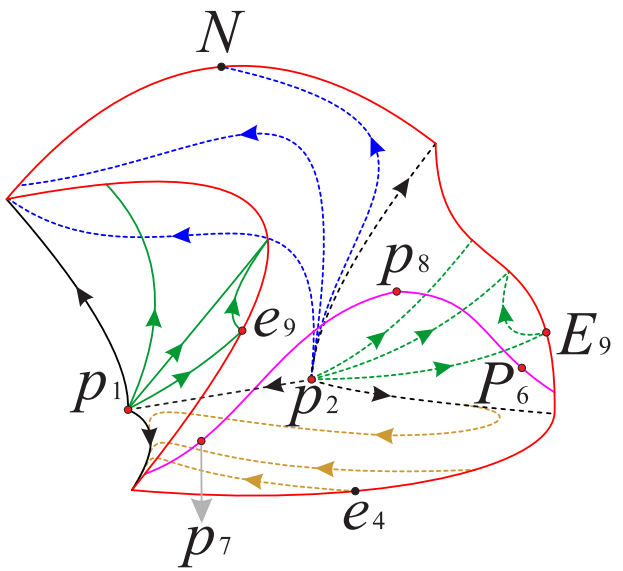

(e)

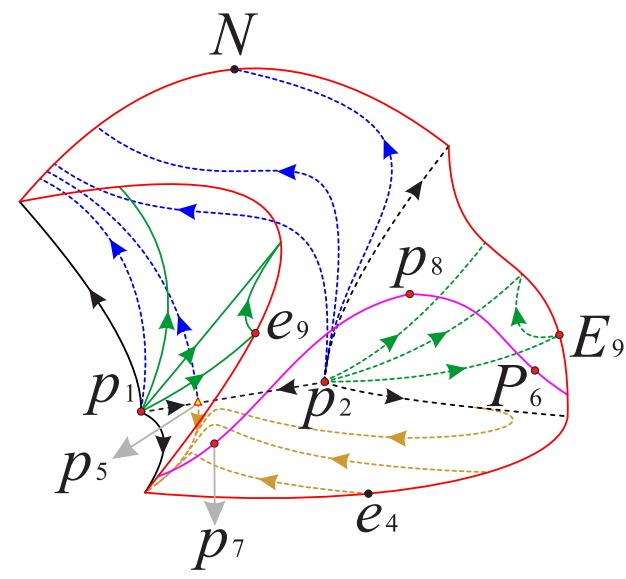

(b)

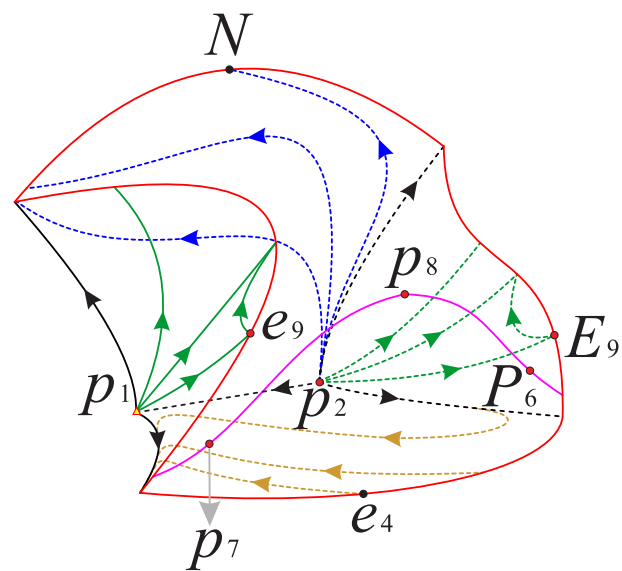

(d)

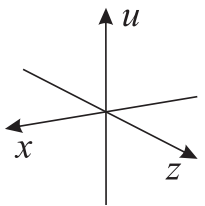

Figure 15. Phase portrait in the boundary of the region $R_{1}$ for different values of $s$ : (a) $0<s<1$, (b) $s=1$, (c) $1<s<\sqrt{6} / 2$, (d) $s=\sqrt{6} / 2$, (e) $s>\sqrt{6} / 2$. The surfaces above and below the long dashed line represent regions I and III, respectively. 


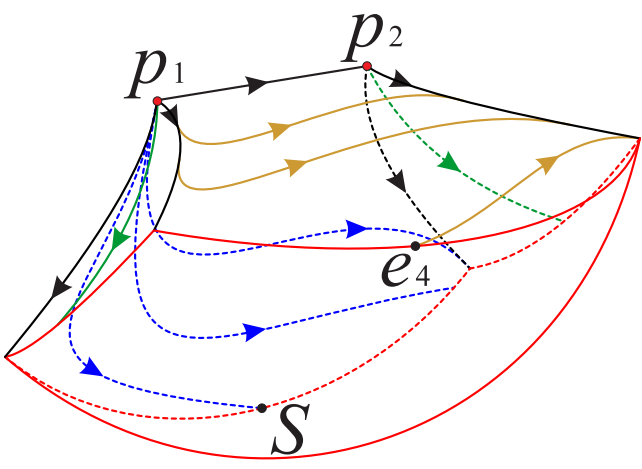

(a)

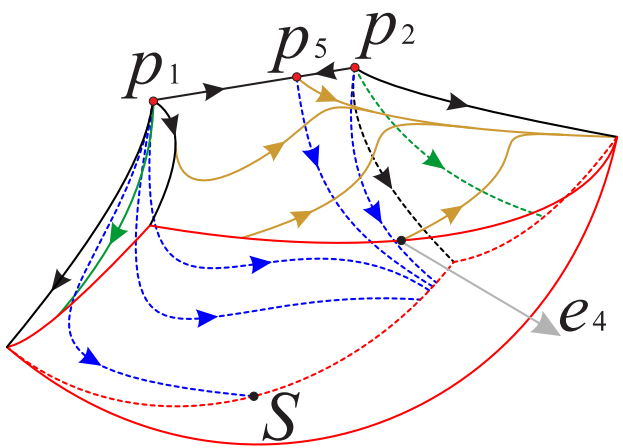

(c)

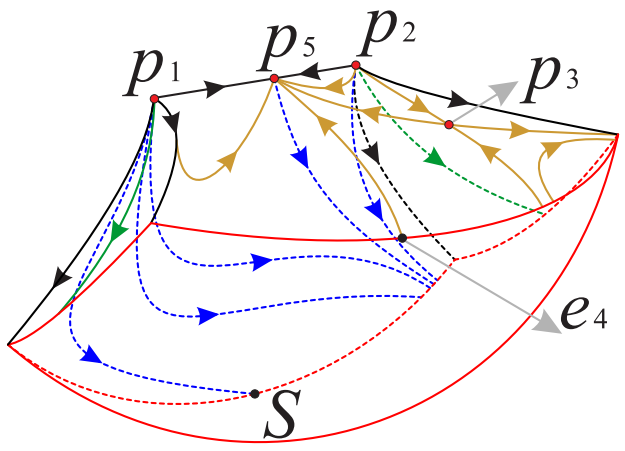

(e)

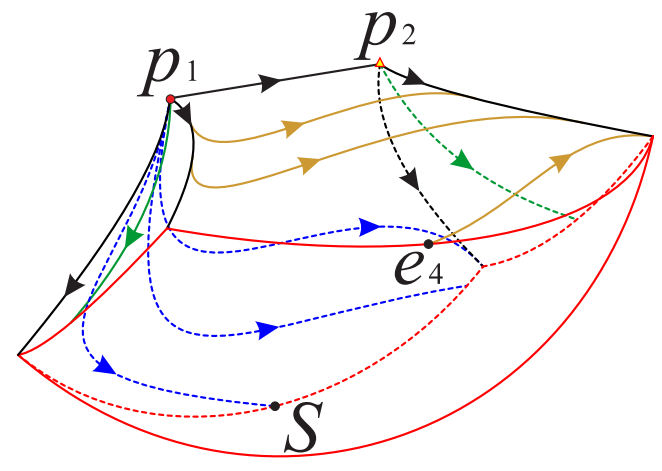

(b)

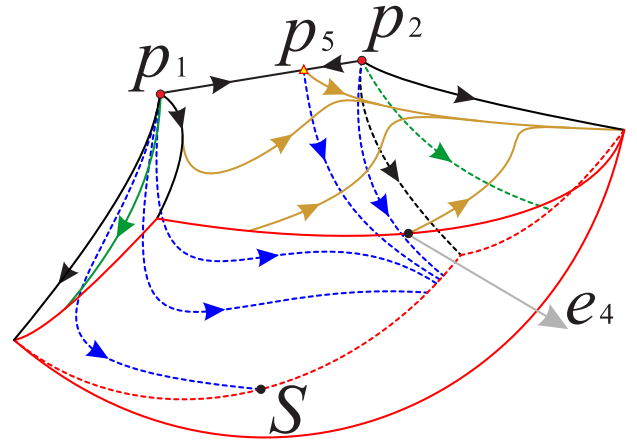

(d)

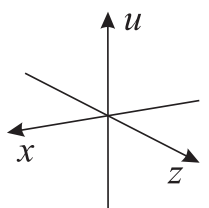

Figure 16. Phase portrait in the boundary of the region $R_{2}$ for different values of $s$ : (a) $s<-\sqrt{6} / 2$ (b) $s=-\sqrt{6} / 2,(\mathbf{c})-\sqrt{6} / 2<s<-1$, (d) $s=-1$, (e) $-1<s<0$. The surfaces above and below the long dashed line represent regions I and III, respectively. 


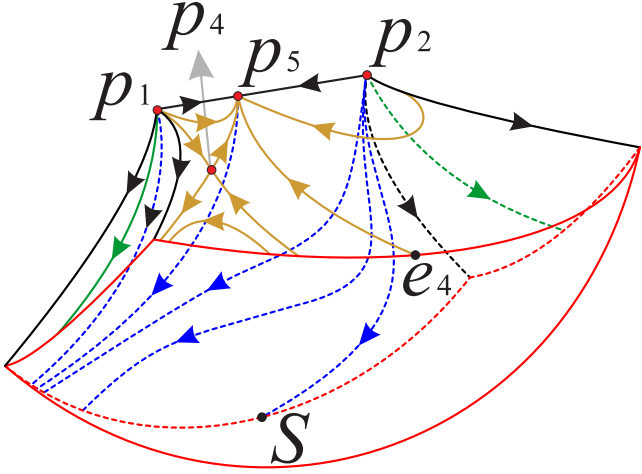

(a)

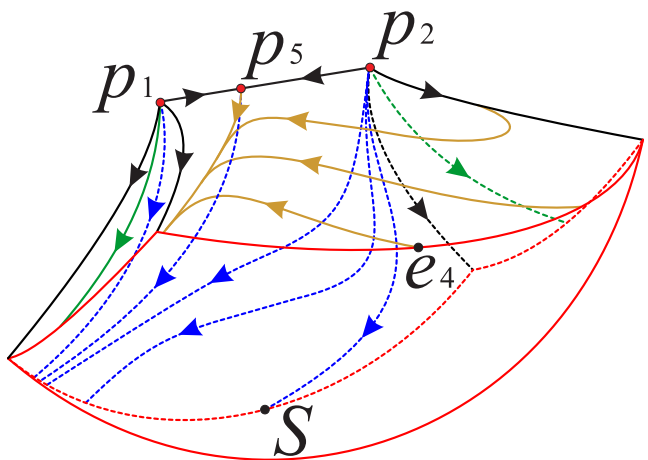

(c)

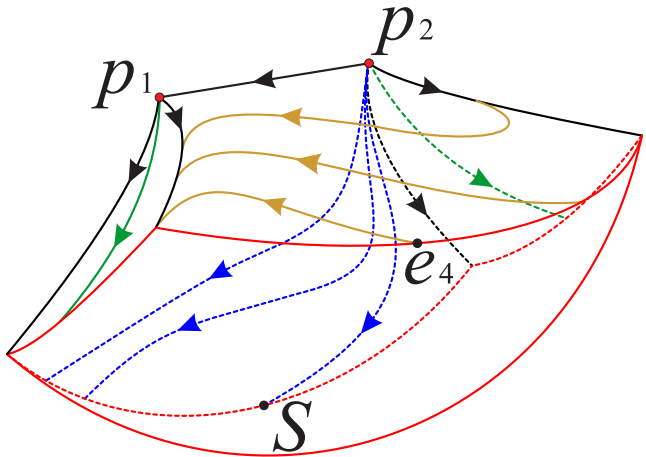

(e)

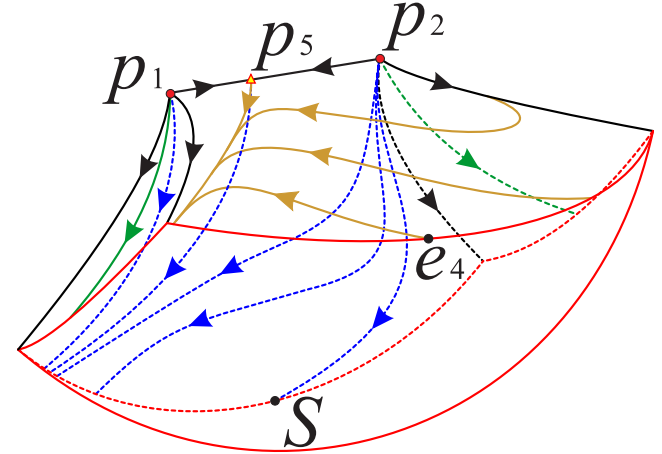

(b)

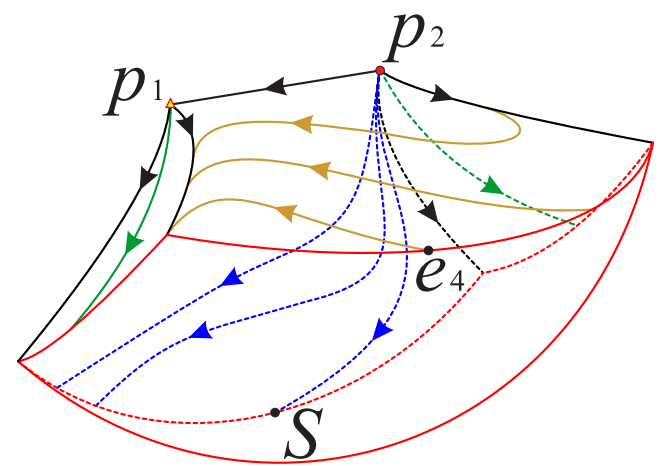

(d)

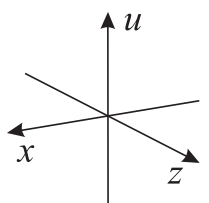

Figure 17. Phase portrait in the boundary of the region $R_{2}$ for different values of $s$ : (a) $0<s<1$, (b) $s=1$, (c) $1<s<\sqrt{6} / 2$, (d) $s=\sqrt{6} / 2$, (e) $s>\sqrt{6} / 2$. The surfaces above and below the long dashed line represent regions I and III, respectively.

Now as shown in Figures 18 and 19, we divided the boundary of the regions $R_{1}$ and $R_{2}$ into sub-surfaces $B_{11}, B_{12}, \cdots$ and $B_{15}$; and $B_{21}, B_{22}, \cdots, B_{25}$, respectively. Thus, the phase portrait on the boundary of $R_{1}$ could be displayed more clearly. Therefore, we can find from Figures 13, 15 and 17 that there is a hyperbolic sector at the north pole $N$ on spherical boundary $B_{11}$ of the Poincaré ball, and $N$ is stable on the back boundary plane $B_{12}$. The equilibrium points $p_{1}$ and $p_{2}$ are unstable on the boundary surfaces $B_{l_{1}} l_{2}$ $\left(l_{1}=1,2, l_{2}=2,3,4\right)$. Moreover, the equilibrium point $p_{5}$ is unstable on the back boundary planes $B_{12}$ and $B_{22}$, and it is stable on the bottom boundary planes $B_{13}$ and $B_{23}$ and at their intersection. In addition the properties of the remaining equilibrium points that are not located at the intersection of these boundary surfaces and planes have been studied in the previous section and will not be repeated here. 


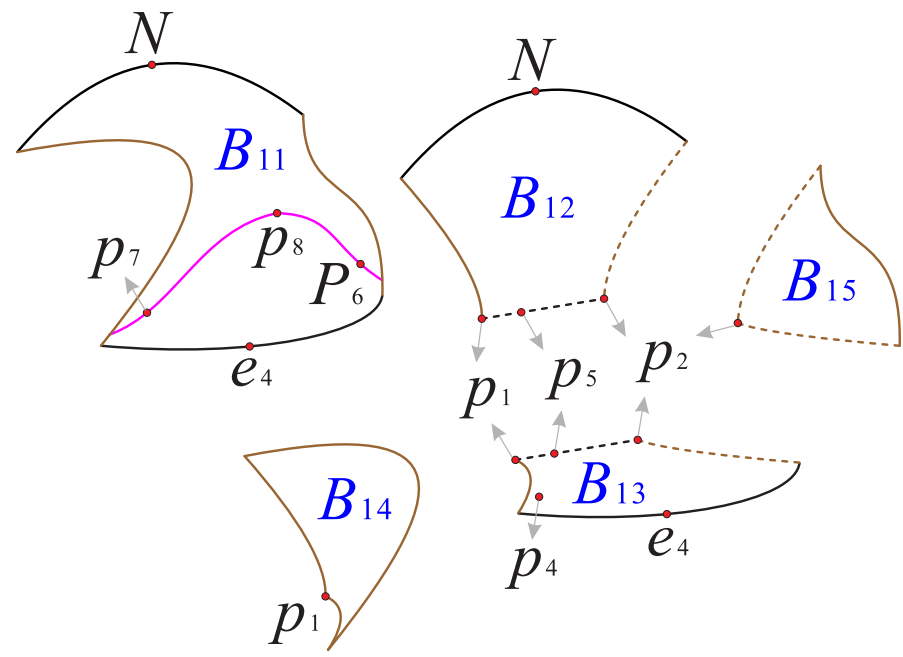

Figure 18. The three boundary surfaces and two boundary planes of the region $R_{1}$.

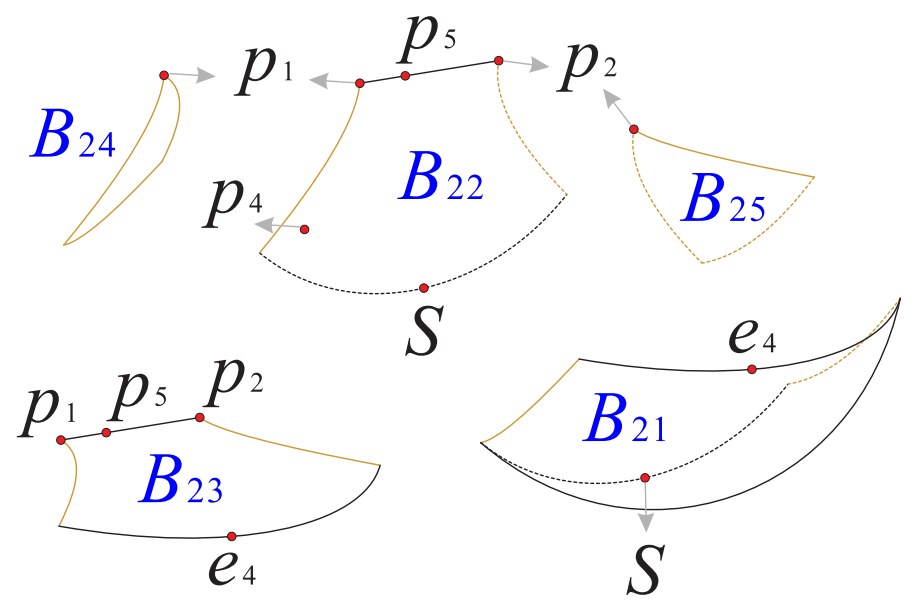

Figure 19. The three boundary surfaces and two boundary planes of the region $R_{2}$.

\subsection{Dynamics in the Interior of the Regions $R_{1}$ and $R_{2}$}

Without loss of generality and considering the physical region of interest, we take $s=\sqrt{6} / 4$, and the dynamics of system (7) can be studied in the same way when we take other values of $s$. Then the five finite equilibrium points of system $(7)$ have the form $p_{1}=\{1,0,0\}, p_{2}=\{-1,0,0\}, p_{3}=\{4 / 3,-\sqrt{15} / 3,0\}, p_{4}=\{4 / 3, \sqrt{15} / 3,0\}$ and $p_{5}=\{1 / 2,0,0\}$. The dynamical behavior of system (7) inside the region $R_{1}$ is determined by the behavior of the flow in the following planes and surfaces:

$$
\begin{aligned}
& z=0, u=0, f_{+}(x, z, u)=0, \\
& h_{1}(x, z, u)=0, h_{2}(x, z, u)=0, h_{3}(x, z, u)=0,
\end{aligned}
$$

where

$$
\begin{aligned}
& h_{1}(x, z, u)=\sqrt{6} s\left[-x^{2}+(u-z)^{2}+1\right]+x\left[3 x^{2}+2(u-z) z-3\right], \\
& h_{2}(x, z, u)=3 x^{2}+2(u-z) z-2, \\
& h_{3}(x, z, u)=3 x^{2}+2(u-z) z .
\end{aligned}
$$

The above planes and surfaces divide the regions $R_{1}$ and $R_{2}$ into eleven subregions $R_{1 i}, i=(1, \ldots, 11)$ and nine subregions $R_{2 j}, j=(1, \ldots, 9)$, respectively; see Figures 20-23 for more details. The signs of the functions $h_{1}, h_{2}$ and $h_{3}$ in these subregions of $R_{1}$ and $R_{2}$ can be found in Tables 4 and 5, respectively. 

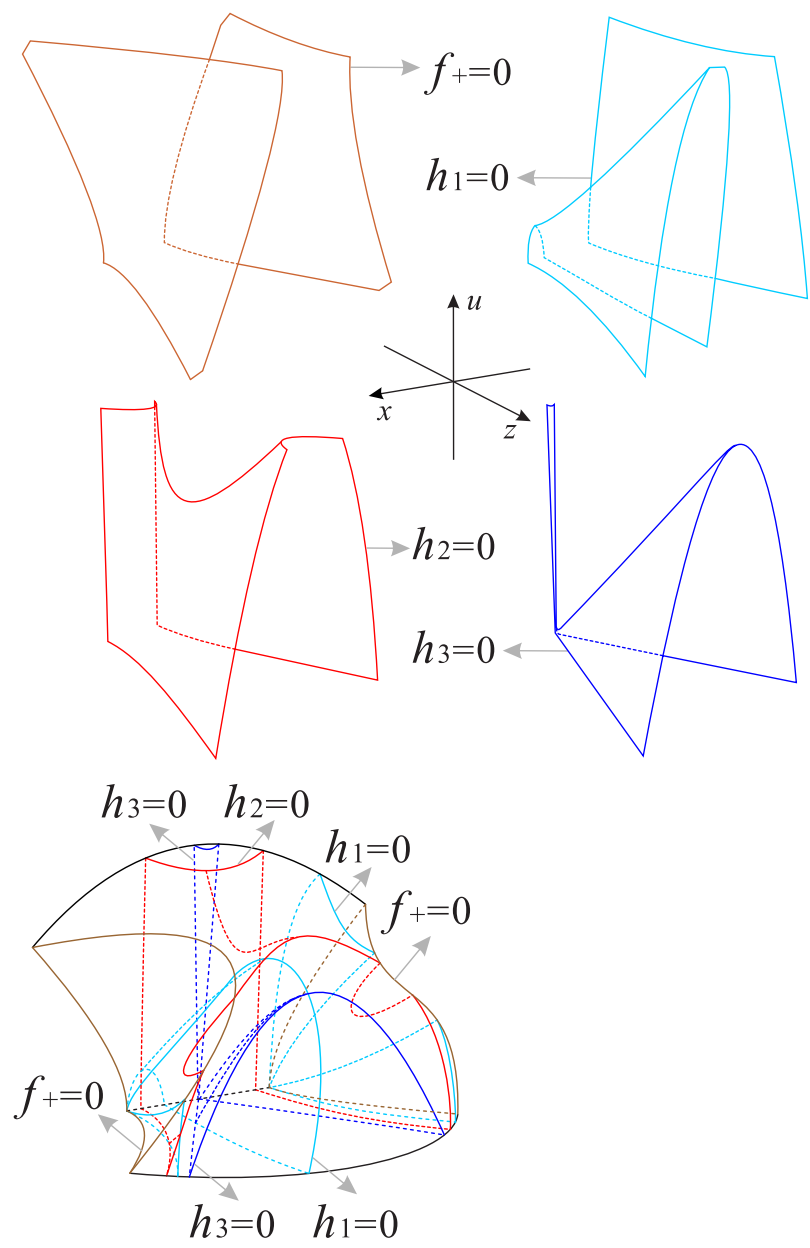

Figure 20. The surfaces $h_{1}, h_{2}$ and $h_{3}$ restricted to the invariant surface $f_{+}(x, z, u)=0$ and the region $R_{1}$ of the Poincaré ball.
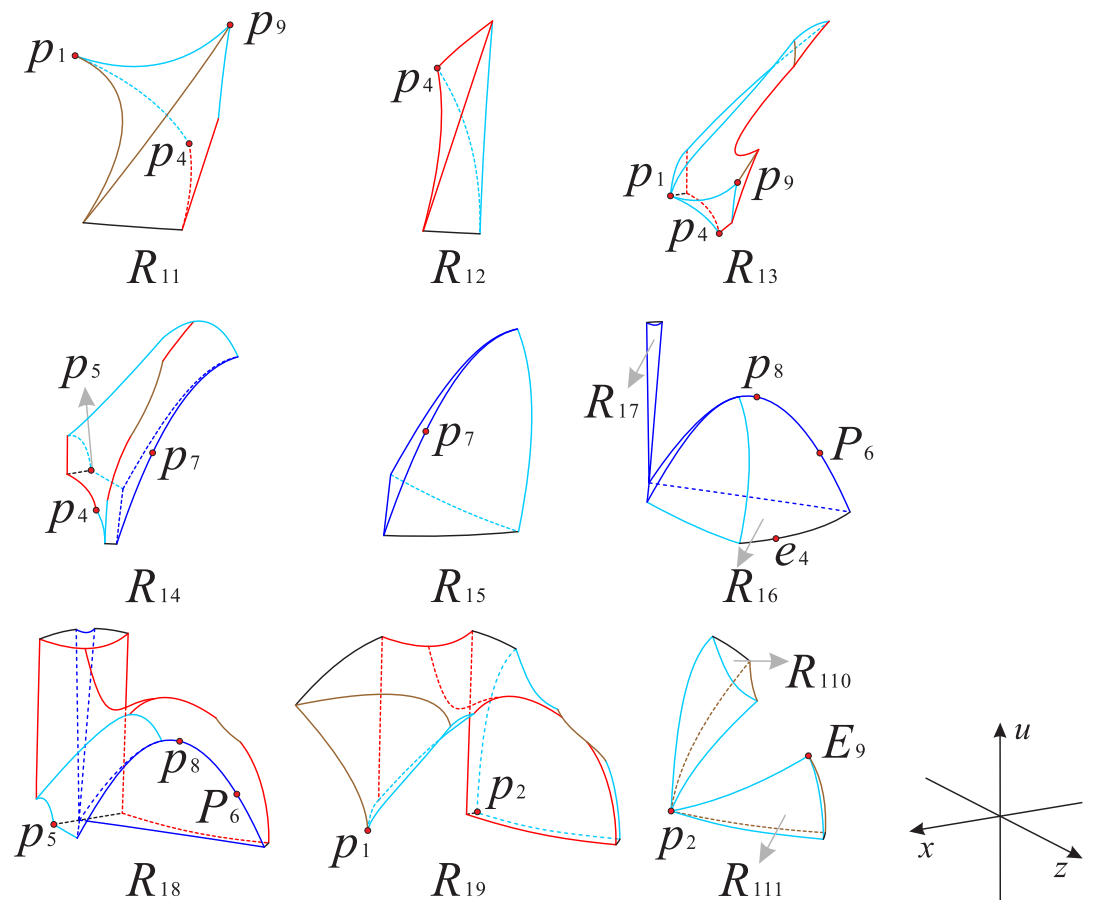

Figure 21. There are the eleven subregions inside the region $R_{1}$ of the Poincaré ball. 


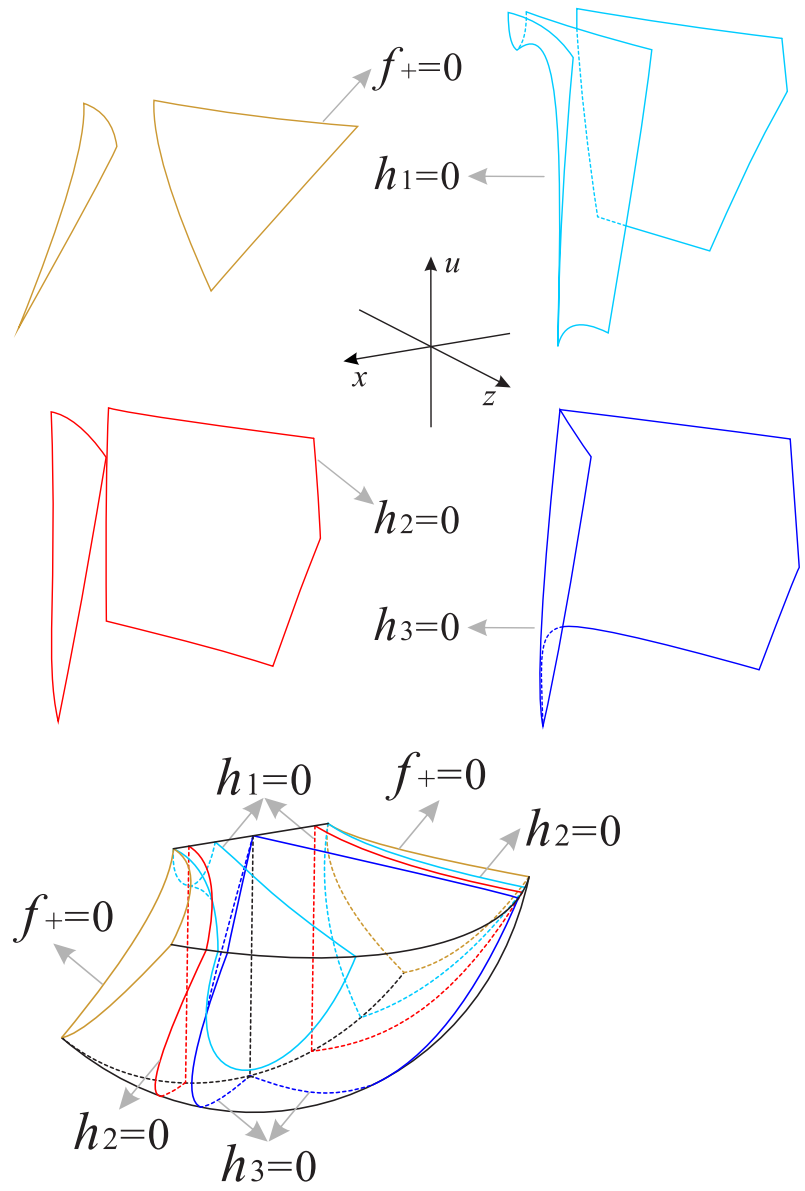

Figure 22. The surfaces $h_{1}, h_{2}$ and $h_{3}$ restricted to the invariant surface $f_{+}(x, z, u)=0$ and the region $R_{2}$ of the Poincaré ball.

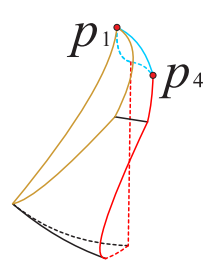

$R_{21}$

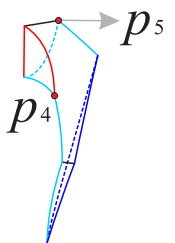

$R_{24}$

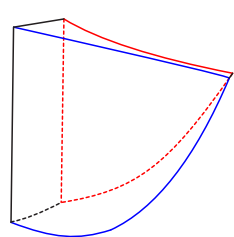

$R_{27}$

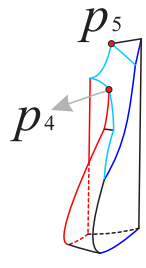

$R_{22}$

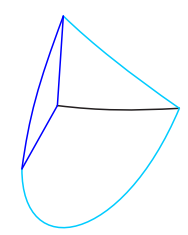

$R_{25}$

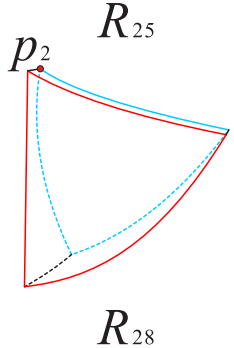

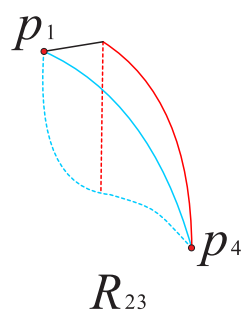

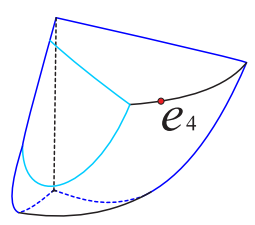

$R_{26}$

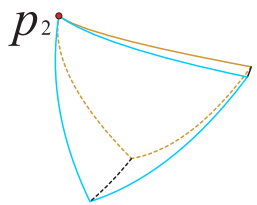

$R_{29}$

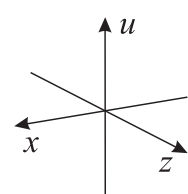

Figure 23. There are the nine subregions inside the region $R_{2}$ of the Poincaré ball. 
Table 4. Signs of functions $h_{1}, h_{2}$ and $h_{3}$ in the subregions of $R_{1}$.

\begin{tabular}{cll}
\hline Functions & Positive & Negative \\
\hline$h_{1}$ & $R_{11}, R_{12}, R_{16}, R_{17}, R_{18}, R_{19}$ & $R_{13}, R_{14}, R_{15}, R_{110}, R_{111}$ \\
\hline$h_{2}$ & $R_{11}, R_{13}, R_{19}, R_{110}, R_{111}$ & $R_{12}, R_{14}, R_{15}, R_{16}, R_{17}, R_{18}$ \\
\hline$h_{3}$ & $R_{11}, R_{12}, R_{13}, R_{14}, R_{18}, R_{19}, R_{110}, R_{111}$ & $R_{15}, R_{16}, R_{17}$ \\
\hline
\end{tabular}

Table 5. Signs of functions $h_{1}, h_{2}$ and $h_{3}$ in the subregions of $R_{2}$.

\begin{tabular}{cll}
\hline Functions & Positive & Negative \\
\hline$h_{1}$ & $R_{21}, R_{22}, R_{26}, R_{27}, R_{28}$ & $R_{23}, R_{24}, R_{25}, R_{29}$ \\
\hline$h_{2}$ & $R_{21}, R_{23}, R_{28}, R_{29}$ & $R_{22}, R_{24}, R_{25}, R_{26}, R_{27}$ \\
\hline$h_{3}$ & $R_{21}, R_{22}, R_{23}, R_{24}, R_{27}, R_{28}, R_{29}$ & $R_{25}, R_{26}$ \\
\hline
\end{tabular}

In addition it should also be noted that in order to avoid visual confusion, we change the dashed lines and solid lines in Figures 21 and 23 to the normal perspective, instead of corresponding to the dashed lines and solid lines in Figures 20 and 22, respectively. What we need to pay attention here is that for any value of $s$, the finite equilibrium point $p_{4}$ is located at the intersection of the surfaces $h_{1}$ and $h_{2}$ on the invariant plane $u=0$, and the finite equilibrium point $p_{5}$ is located at the intersection of the surface $h_{1}$ and $x$-axis (see Figures 21 and 23). For the infinite equilibrium point $p_{7}=(3 / 2,1 / 2,0)$ of Equations (25), according to the three-dimensional Poincaré transformation in the local chart $U_{1}$, we know that $p_{7}=\left(1 / z_{3}, 3 /\left(2 z_{3}\right), 1 /\left(2 z_{3}\right)\right)\left(z_{3} \rightarrow 0\right)$ is on the intersection of the surface $h_{3}$ and the Poincare sphere. Similarly, in the $x z u$ coordinate system we have $p_{6}=\left(1 / z_{3},-3 /\left(2 z_{3}\right),-1 /\left(2 z_{3}\right)\right)\left(z_{3} \rightarrow 0\right)$, since the infinite equilibrium points $P_{6}$ and $p_{6}$ are symmetric about the origin, then we obtain $P_{6}=$ $\left(-1 / z_{3}, 3 /\left(2 z_{3}\right), 1 /\left(2 z_{3}\right)\right)\left(z_{3} \rightarrow 0\right)$, which is symmetric with $p_{7}$ with respect to the plane $x=0$. For the infinite equilibrium point $e_{9}=(-1 / 2,0)$ of equations (20) when $u \geq z$, we combine the relationship $x= \pm \sqrt{1+y^{2}}$ in equations (18) and the invariant surface $f_{+}(x, z, u)=0$ to know that the coordinate of $e_{9}$ in the three-dimensional coordinate system $x z u$ can be denoted as $p_{9}=\left(\sqrt{1+V^{2}} / V, 1 /(2 V),-1 /(2 V)\right)(V \rightarrow 0)$, obviously the infinite equilibrium point $p_{9}$ is not in the region $R_{1}$. However, when $u \leq z$ we have $p_{9}=\left(\sqrt{1+V^{2}} / V, 3 /(2 V), 1 /(2 V)\right)(V \rightarrow 0)$, and it is exactly located on the surface $h_{1}$, on the invariant surface $f_{+}(x, z, u)=0$ and in their intersection with the Poincaré sphere (see Figure 21).

As it is shown in the subregion $R_{11}$ (see Figure 21) the left side surface is contained in the invariant surface $f_{+}(x, z, u)=0$, the bottom plane is contained in the invariant plane $u=0$, the right-back segment surface is contained in $h_{1}=0$ and the right-front segment surface is contained in $h_{2}=0$. From Table 6 we find that the orbits of system (7) increase monotonically along the positive directions of the three coordinate axes, which means that the orbits in $R_{11}$ come from the finite equilibrium points $p_{1}$ and $p_{4}$, or from the subregion $R_{12}$, and then go to the boundary of Poincaré sphere restricted to this subregion.

In the subregion $R_{12}$, its bottom plane is contained in the invariant plane $u=0$, the left side surface is contained in the surface $h_{2}=0$ and the right side surface is contained in the surface $h_{1}=0$. Then we can see that the orbits of system (7) in this subregion monotonically decrease along the positive direction of the $z$-axis, but increase monotonically along the positive direction of the $x$-axis and $u$-axis, so the orbits start at the infinite equilibrium points on the Poincaré sphere in this subregion and then enter into the subregion $R_{11}$.

The bottom plane in the subregion $R_{13}$ is contained in the invariant plane $u=0$, the left side surface is contained in the surface $h_{1}=0$, the right side surface is contained in the surface $h_{2}=0$ and the backplane is contained in the invariant plane $z=0$. The upper and lower surfaces of the front are composed of the intersection of the surfaces $h_{1}=0$ and $h_{2}=0$ and the invariant surface $f_{+}(x, z, u)=0$ on the Poincare sphere. The orbits in this subregion increase monotonically along the positive $z$-axis and $u$-axis, but decrease monotonically along the positive $x$-axis, so the orbits start at the finite point $p_{1}$, 
and then cross the side surfaces of this subregion and eventually go to the subregion $R_{14}$, respectively.

Table 6. Dynamical behavior in the twenty subregions.

\begin{tabular}{cll}
\hline Subregions & Corresponding Region & Increase or Decrease \\
\hline$R_{11}$ & $h_{1}>0, h_{2}>0, h_{3}>0, z>0, u>0$ & $\dot{x}>0, \dot{z}>0, \dot{u}>0$ \\
\hline$R_{12}$ & $h_{1}>0, h_{2}<0, h_{3}>0, z>0, u>0$ & $\dot{x}>0, \dot{z}<0, \dot{u}>0$ \\
\hline$R_{13}$ & $h_{1}<0, h_{2}>0, h_{3}>0, z>0, u>0$ & $\dot{x}<0, \dot{z}>0, \dot{u}>0$ \\
\hline$R_{14}$ & $h_{1}<0, h_{2}<0, h_{3}>0, z>0, u>0$ & $\dot{x}<0, \dot{z}<0, \dot{u}>0$ \\
\hline$R_{15}$ & $h_{1}<0, h_{2}<0, h_{3}<0, z>0, u>0$ & $\dot{x}<0, \dot{z}<0, \dot{u}<0$ \\
\hline$R_{16}$ & $h_{1}>0, h_{2}<0, h_{3}<0, z>0, u>0$ & $\dot{x}>0, \dot{z}<0, \dot{u}<0$ \\
\hline$R_{17}$ & $h_{1}>0, h_{2}<0, h_{3}<0, z>0, u>0$ & $\dot{x}>0, \dot{z}<0, \dot{u}<0$ \\
\hline$R_{18}$ & $h_{1}>0, h_{2}<0, h_{3}>0, z>0, u>0$ & $\dot{x}>0, \dot{z}<0, \dot{u}>0$ \\
\hline$R_{19}$ & $h_{1}>0, h_{2}>0, h_{3}>0, z>0, u>0$ & $\dot{x}>0, \dot{z}>0, \dot{u}>0$ \\
\hline$R_{110}$ & $h_{1}<0, h_{2}>0, h_{3}>0, z>0, u>0$ & $\dot{x}<0, \dot{z}>0, \dot{u}>0$ \\
\hline$R_{111}$ & $h_{1}<0, h_{2}>0, h_{3}>0, z>0, u>0$ & $\dot{x}<0, \dot{z}>0, \dot{u}>0$ \\
\hline$R_{21}$ & $h_{1}>0, h_{2}>0, h_{3}>0, z>0, u<0$ & $\dot{x}>0, \dot{z}>0, \dot{u}<0$ \\
\hline$R_{22}$ & $h_{1}>0, h_{2}<0, h_{3}>0, z>0, u<0$ & $\dot{x}>0, \dot{z}<0, \dot{u}<0$ \\
\hline$R_{23}$ & $h_{1}<0, h_{2}>0, h_{3}>0, z>0, u<0$ & $\dot{x}<0, \dot{z}>0, \dot{u}<0$ \\
\hline$R_{24}$ & $h_{1}<0, h_{2}<0, h_{3}>0, z>0, u<0$ & $\dot{x}<0, \dot{z}<0, \dot{u}<0$ \\
\hline$R_{25}$ & $h_{1}<0, h_{2}<0, h_{3}<0, z>0, u<0$ & $\dot{x}<0, \dot{z}<0, \dot{u}>0$ \\
\hline$R_{26}$ & $h_{1}>0, h_{2}<0, h_{3}<0, z>0, u<0$ & $\dot{x}>0, \dot{z}<0, \dot{u}>0$ \\
\hline$R_{27}$ & $h_{1}>0, h_{2}<0, h_{3}>0, z>0, u<0$ & $\dot{x}>0, \dot{z}<0, \dot{u}<0$ \\
\hline$R_{28}$ & $h_{1}>0, h_{2}>0, h_{3}>0, z>0, u<0$ & $\dot{x}>0, \dot{z}>0, \dot{u}<0$ \\
\hline$R_{29}$ & $h_{1}<0, h_{2}>0, h_{3}>0, z>0, u<0$ & $\dot{x}<0, \dot{z}>0, \dot{u}<0$ \\
\hline
\end{tabular}

The bottom plane of the subregion $R_{14}$ is contained in the invariant plane $u=0$; the left surface is contained in the surface composed of the surfaces $h_{1}=0$ and $h_{2}=0$; the invariant plane $f_{+}(x, z, u)=0$ and the right surface are contained in the surface $h_{3}=0$; the back surface is contained in the surface $h_{1}=0$; the backplane is contained in the invariant plane $z=0$; the front surface is contained in the surface enclosed by the intersection lines of the surfaces $h_{1}=0, h_{2}=0$ and $h_{3}=0$, and the invariant surface $f_{+}(x, z, u)=0$ on the Poincaré sphere. The orbits in this subregion increase monotonically along the positive $u$-axis, and decrease monotonically along the positive two other coordinate axes, which indicates that the orbits of this subregion start from the finite point $p_{4}$ or the equilibrium points on the Poincaré sphere at infinity in this subregion, or come from the subregion $R_{13}$ and finally enter into subregions $R_{15}$ or $R_{18}$.

The bottom plane of the subregion $R_{15}$ is contained in the invariant plane $u=0$, the front surface is contained in the Poincaré sphere, the left surface is contained in the surface $h_{3}=0$ and the right surface is contained in the surface $h_{1}=0$. The dynamic behavior of the orbits in this subregion is the same as that in the subregion $R_{14}$, and they decrease monotonically along the positive directions of the three coordinate axes, which means that the orbits of start from subregion $R_{14}$ or the infinite equilibrium points on the Poincaré sphere in this subregion, and then enter into subregion $R_{16}$.

The bottom plane of the subregion $R_{16}$ is contained in the invariant plane $u=0$, the left and right surfaces are contained in the surface $h_{3}=0$, the front left surface is contained in the surface $h_{1}=0$ and the front right surface is contained in Poincaré sphere. The orbits in this subregion increase monotonically along the positive $x$-axis, and decrease monotonically along the positive two other coordinate axes; thereby the orbits originate from the infinite equilibrium points on the Poincaré sphere in this subregion, and then enter into the subregion $R_{18}$. 
The front surface of the subregion $R_{17}$ is contained in the surface $h_{3}=0$, and the back surface is contained in the invariant plane $z=0$. The dynamic behavior of the orbits in this subregion is the same as in the subregion $R_{16}$, they come from the subregion $R_{18}$, after crossing the right part boundary surface of the subregion $R_{17}$ and then from its left boundary surface back to the subregion $R_{18}$.

The bottom plane of the subregion $R_{18}$ is contained in the invariant plane $u=0$; the left surface is contained in the surface $h_{1}=0$; the upper surface and the right surface are contained in the surface $h_{2}=0$ and the invariant surface $f_{+}(x, z, u)=0$; the front surface is contained in the Poincare sphere; the surface below the front surface is contained in the surface $h_{3}=0$; and the back surface is composed of the invariant plane $z=0$ and the surface $h_{3}=0$. The orbits in this subregion decrease monotonically in the positive direction along the $z$-axis, and decrease monotonically in the positive direction along the other two coordinate axes, for this reason the orbits start at the equilibrium points on the Poincaré sphere at infinity or come from the subregions $R_{14}, R_{16}$, the left side part surface of $R_{17}$, and then tend to the subregion $R_{19}$.

The backplane of the subregion $R_{19}$ is divided into the left and right parts by the surface $h_{2}=0$, but both of them are contained in the invariant plane $z=0$. The left and right side surfaces of $R_{19}$ are contained in the surface composed of the surface $h_{1}=0$ and the invariant surface $f_{+}(x, z, u)=0$; the top surface is contained in the Poincare sphere; the lower-right surface is contained in the surface $h_{2}=0$; and the lower-left surface is contained in the surface $h_{1}=0$. The orbits in this subregion are monotonically increasing along the positive direction of the three coordinate axes, so the orbits come from the finite equilibrium points $p_{1}$ and $p_{2}$, or from the subregion $R_{18}$, and finally approach the equilibrium points on the Poincaré sphere at infinity.

The subregions $R_{110}$ and $R_{111}$ are connected by the finite equilibrium point $p_{2}$; their front surfaces are contained in the surface $h_{1}=0$; the right surface of $R_{110}$ and the back surface of $R_{111}$ are contained in the invariant surface $f_{+}(x, z, u)=0$; the back surface of $R_{110}$ is contained in the invariant plane $z=0$; the top surface of $R_{110}$ and the surface on the right side of $R_{111}$ are contained in the Poincaré sphere. The orbits in these two subregions decrease monotonically along the positive direction of the $x$-axis, and monotonically increase along the positive direction along the other two coordinate axes. Thus, the orbits in these two subregions start at the equilibrium point $p_{2}$, and then eventually go to the corresponding infinite equilibrium point on the Poincaré sphere.

Therefore, the dynamic process of the orbits inside the eleven subregions of $R_{1}$ discussed above can be summarized as

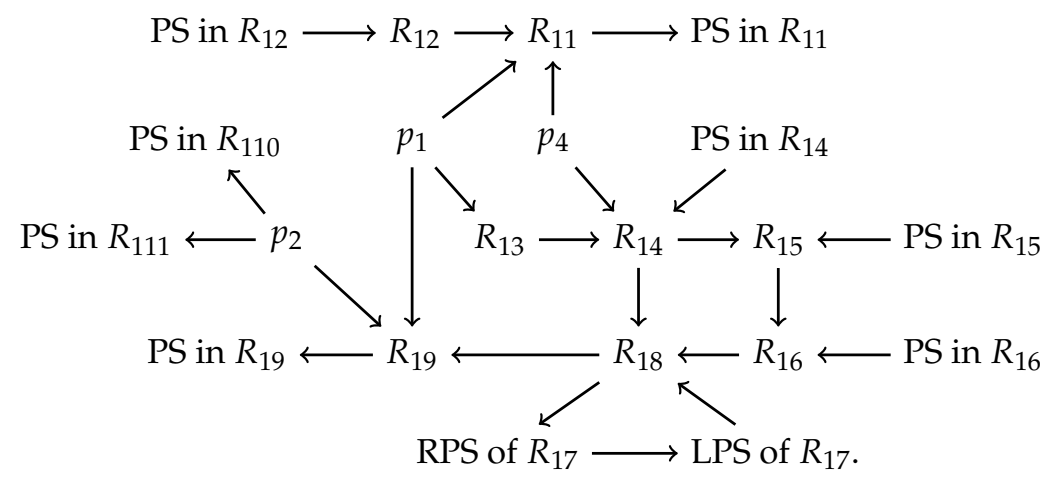

Note: PS represents Poincaré sphere. RPS denotes the right part of surface. LPS stands for the left part of surface.

The flow chart discussed above shows that the orbits of system (7) contained in the region $R_{1}$ have an $\omega$-limit in the subregions $R_{11}, R_{19}, R_{110}$ and $R_{111}$ when these four subregions are restricted to the Poincare sphere at infinity. Furthermore, the orbits have an $\alpha$-limit at the finite equilibrium points $p_{1}, p_{2}$ and $p_{4}$. In addition the orbits also have 
an $\omega$-limit in the subregions $R_{12}, R_{14}, R_{15}$ and $R_{16}$ when they are confined to the Poincaré sphere at infinity.

For the subregions in $R_{2}$ (see Figure 23), the front and bottom surfaces of the subregion $R_{21}$ are contained in the Poincaré sphere, the back surface is contained in the invariant plane $z=0$, the top surface is contained in the invariant plane $u=0$, the left surface is contained in the invariant plane $f_{+}(x, z, u)=0$, the lower right surface is contained in the surface $h_{2}=0$ and the upper right surface is contained in the surface $h_{1}=0$. The orbits in this subregion increase monotonically along the positive direction of the $x$-axis and $z$-axis, and the monotonically decrease along the positive direction of the $u$-axis, so the orbits in this subregion start from the finite equilibrium points $p_{1}, p_{4}$ and the right surface $h_{2}=0$, and finally approaching the point of infinity at Poincaré sphere.

The front and bottom surfaces of the subregion $R_{22}$ are contained in the Poincaré sphere, the top plane is divided into two parts by the surface $h_{1}=0$, but these two parts are contained in the same invariant plane $u=0$, the left side surface is contained in the surface $h_{2}=0$, the right side surface is contained in the surface $h_{3}=0$ and the back surface is contained in the invariant plane $z=0$. The orbits in this subregion increase monotonically along the positive direction of the $x$-axis, and decrease along the positive direction of the other two axes. Therefore, the orbits in this subregion start from the connected subregions $R_{24}$ and $R_{26}$, they enter into this subregion through the surfaces $h_{1}=0$ and $h_{3}=0$, and then go through the surface $h_{2}=0$ into the subregion $R_{21}$.

The top and back planes of the subregion $R_{23}$ are contained in the invariant plane $u=0$ and the invariant plane $z=0$, respectively; the left surface is contained in the surface $h_{1}=0$; and the right surface is contained in the surface $h_{2}=0$. The orbits in this subregion increase monotonically along the positive direction of the $z$-axis and decrease monotonically along the positive direction of the other two axes, which indicates that the orbits in this subregion start at the finite equilibrium point $p_{1}$ and cross the surface $h_{2}=0$, and then enter into the subregion $R_{24}$.

The top and back planes of the subregion $R_{24}$ are also contained in the invariant plane $u=0$ and the invariant plane $z=0$, respectively; the bottom surface is contained in the surface $h_{1}=0$; the left surface is contained in the surface $h_{2}=0$; the right surface is contained in the surface $h_{3}=0$; and the front surface is contained in the Poincare sphere. Then the orbits in this subregion are monotonically decreasing along the positive direction of the three axes, which means that the orbits in this subregion start at the finite equilibrium point $p_{4}$, or come from the subregion $R_{23}$ and the infinite equilibrium points on the Poincaré sphere, and then directly enter into the subregions $R_{22}$ and $R_{25}$ through the surfaces $h_{1}=0$ and $h_{3}=0$, respectively.

The top plane of the subregion $R_{25}$ is contained in the invariant plane $u=0$, the bottom surface is contained in the surface $h_{1}=0$, the left surface is contained in the surface $h_{3}=0$ and the front surface is contained in the Poincare sphere. The orbits in this subregion are monotonically decreasing along the positive direction of the $x$-axis and $z$-axis, but monotonically increasing along the positive direction of the $u$-axis, which indicates that the orbits in this subregion start from the infinity equilibrium point on the Poincaré sphere and then cross the surface $h_{1}=0$ into the subregion $R_{26}$.

The top plane of the subregion $R_{26}$ is also contained in the invariant plane $u=0$, the right and bottom surfaces are contained in the Poincare sphere, the front surface is contained in the surface $h_{1}=0$ and the back-left and back-right surfaces are contained in the surface $h_{3}=0$. The orbits in this subregion are monotonically decreasing along the positive direction of the $z$-axis, but are increasing monotonically along the positive directions of the remaining two axes. Then, the orbits in this subregion start from the subregion $R_{27}$ and cross the back-right surface $h_{3}=0$ or from the infinite equilibrium points on the Poincaré sphere, and then cross the back-left surface $h_{3}=0$ into the subregion $R_{22}$.

The subregion $R_{27}$ consists of the top plane contained in the invariant plane $u=0$, the back plane contained in the invariant plane $z=0$, the back surface contained in the surface $h_{2}=0$, the front surface contained in the surface $h_{3}=0$ and the bottom surface contained 
in the Poincare sphere. The orbits in this subregion are monotonically increasing along the positive $x$-axis, but are monotonically decreasing along the positive directions of the remaining two axes, so they start from the subregion $R_{28}$ or the equilibrium points on the Poincaré sphere at infinity, and then go through the left surface $h_{3}=0$ to the subregion $R_{26}$.

The subregion $R_{28}$ consists of the top plane contained in the invariant plane $u=0$, the left plane contained in the invariant plane $z=0$, the front surface contained in the surface $h_{2}=0$, the back surface contained in $h_{1}=0$ and the right surface contained in the Poincaré sphere. All the orbits in this subregion are monotonically decreasing along the positive direction of the $u$-axis, and monotonically increasing along the positive direction of the other two axes, so they start from the finite equilibrium point $p_{2}$ and then enter into the subregion $R_{27}$ through the front surface $h_{2}=0$.

The top plane of the subregion $R_{29}$ is contained in the invariant plane $u=0$, the left plane is contained in the invariant plane $z=0$, the right surface is contained in the Poincare sphere, the front surface is contained in the surface $h_{1}=0$ and the back surface is contained in the constant surface $f_{+}(x, z, u)=0$. The orbits in this subregion are monotonically increasing along the positive direction of the $z$-axis, and monotonically decreasing along the positive direction of the other two axes, which means that the orbits start at the finite equilibrium point $p_{2}$ and finally tend to the equilibrium points on Poincaré sphere at infinity.

Therefore, the dynamic behavior of the orbits inside the nine subregions of $R_{2}$ discussed above can be represented as

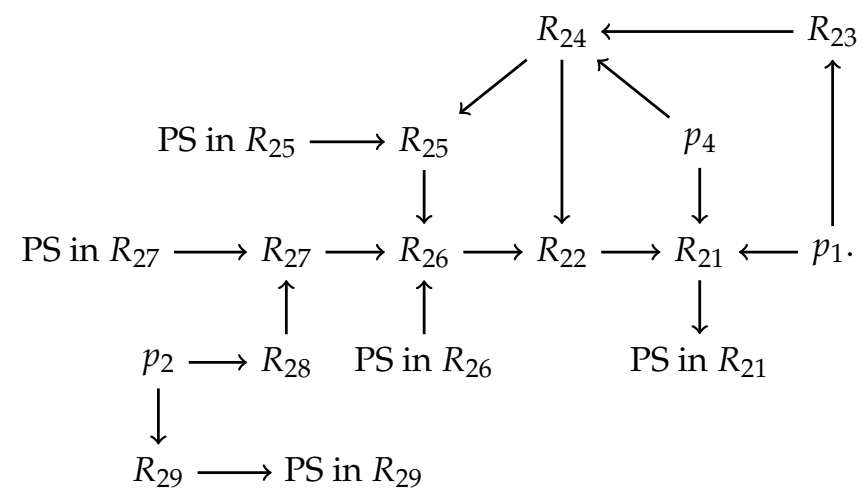

The flow chart in the region $R_{2}$ discussed above shows that the orbits of system (7) contained in this region have an $\alpha$-limit at the finite equilibrium points $p_{1}, p_{2}$ and $p_{4}$; and on the Poincare sphere restricted to the subregions $R_{25}, R_{26}$ and $R_{27}$. Moreover, the orbits have an $\omega$-limit on the Poincaré sphere restricted to the subregions $R_{21}$ and $R_{29}$. Therefore, we fully describe all qualitative global dynamic behaviors of system (7).

\section{Case II: $s \neq 0, k=-1$}

For an open universe the phase portraits of system (7) on the invariant planes $z=0$ and $u=0$ are the same as those in Sections 3.1.1 and 3.1.2. Moreover, the local phase portraits of the finite and infinite equilibrium points on the Poincare sphere are consistent with Sections 3.1.4 and 3.1.5, respectively. However, the physical region of interest $f_{-}(x, z, u)=0$ is no more an invariant surface.

Taking the same approach as in Section 3.2, we also divide the Poincaré ball restricted to the region $x^{2}-(u+z)^{2} \leq 1$ into four regions as below

$$
\begin{aligned}
& S_{1}: z \geq 0, u \geq 0 . \quad S_{2}: z \geq 0, u \leq 0 . \\
& S_{3}: z \leq 0, u \leq 0 . \quad S_{4}: z \leq 0, u \geq 0 .
\end{aligned}
$$

and we shall only study the phase portrait of system (7) in the regions $S_{1}$ and $S_{2}$ due to the symmetry as mentioned before. The phase portrait of system (7) on the boundaries of 
these two regions are the same as the closed universe except for the boundary surfaces $B_{14}$, $B_{15}, B_{24}, B_{25}$ contained on the surface $f_{-}(x, z, u)=0$ (see Figures 14-19).

Dynamics in the Interior of the Regions $S_{1}$ and $S_{2}$

For convenience we continue the discussion of the case $s=\sqrt{6} / 4$. The invariant planes, $z=0$ and $u=0$; and the surfaces, $h_{1}, h_{2}, h_{3}$ and $f_{-}(x, z, u)=0$, divide the regions $S_{1}$ and $S_{2}$ into nine subregions $S_{11}, S_{12}, \cdots, S_{19}$ and ten subregions $S_{21}, S_{22}, \cdots, S_{210}$, respectively; see Figures 24-26 for more details. The signs of the functions $h_{1}, h_{2}$ and $h_{3}$ in these subregions of $S_{1}$ and $S_{2}$ can be found in Tables 7 and 8, respectively.

Table 7. Signs of functions $h_{1}, h_{2}$ and $h_{3}$ in the subregions of $S_{1}$.

\begin{tabular}{cll}
\hline Functions & Positive & Negative \\
\hline$h_{1}$ & $S_{11}, S_{12}, S_{16}, S_{17}, S_{18}$ & $S_{13}, S_{14}, S_{15}, S_{19}$ \\
\hline$h_{2}$ & $S_{11}, S_{13}, S_{19}$ & $S_{12}, S_{14}, S_{15}, S_{16}, S_{17}, S_{18}$ \\
\hline$h_{3}$ & $S_{11}, S_{12}, S_{13}, S_{14}, S_{18}, S_{19}$ & $S_{15}, S_{16}, S_{17}$ \\
\hline
\end{tabular}

Table 8. Signs of functions $h_{1}, h_{2}$ and $h_{3}$ in the subregions of $S_{2}$.

\begin{tabular}{cll}
\hline Functions & Positive & Negative \\
\hline$h_{1}$ & $S_{21}, S_{22}, S_{26}, S_{27}, S_{28}$ & $S_{23}, S_{24}, S_{25}, S_{29}, S_{210}$ \\
\hline$h_{2}$ & $S_{21}, S_{23}, S_{28}, S_{29}, S_{210}$ & $S_{22}, S_{24}, S_{25}, S_{26}, S_{27}$ \\
\hline$h_{3}$ & $S_{21}, S_{22}, S_{23}, S_{24}, S_{27}, S_{28}, S_{29}, S_{210}$ & $S_{25}, S_{26}$ \\
\hline
\end{tabular}

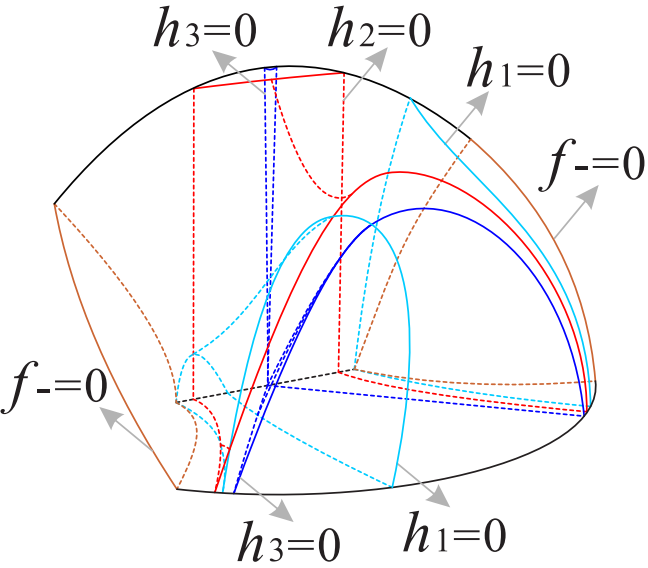

(a) Region $S_{1}$ of the Poincaré ball

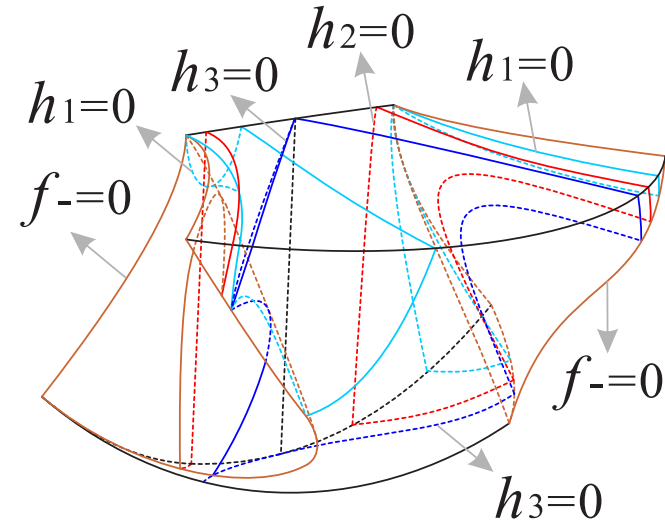

(b) Region $S_{2}$ of the Poincaré ball

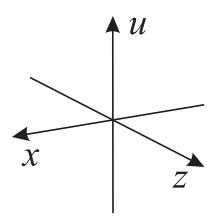

Figure 24. The surfaces $h_{1}, h_{2}$ and $h_{3}$ restricted to the surface $f_{-}(x, z, u)=0$ and the regions $S_{1}$ and $S_{2}$ of the Poincaré ball, respectively. 


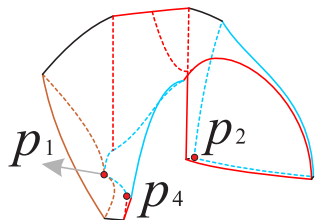

$S_{11}$

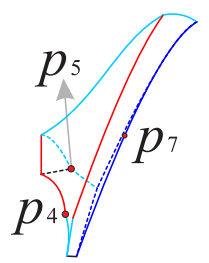

$S_{14}$

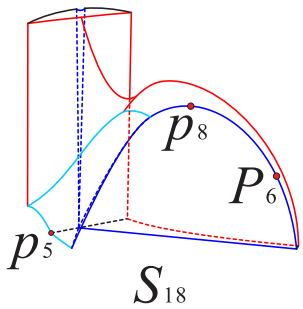

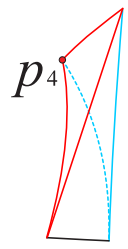

$S_{12}$

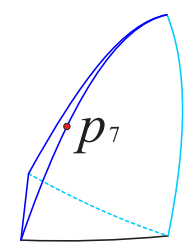

$S_{15}$

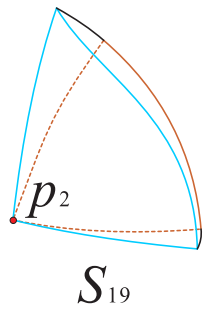

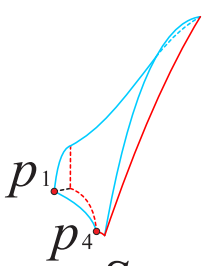

$S_{13}$
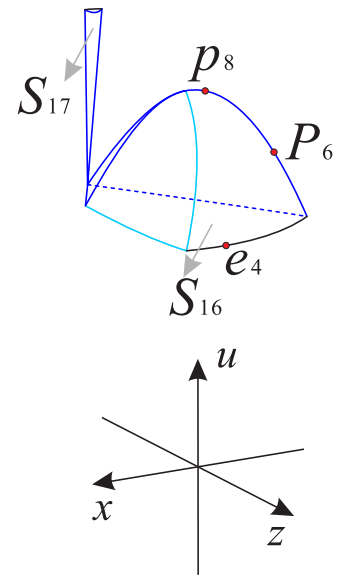

Figure 25. The nine subregions inside the region $S_{1}$ of the Poincaré ball.

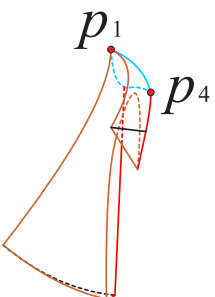

$S_{21}$

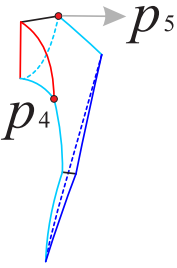

$S_{24}$

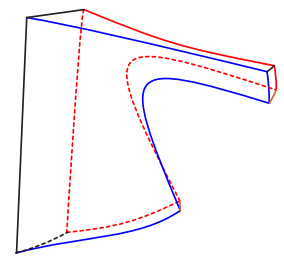

$S_{27}$

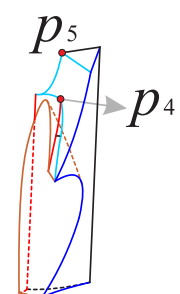

$S_{22}$

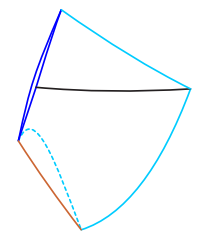

$S_{25}$

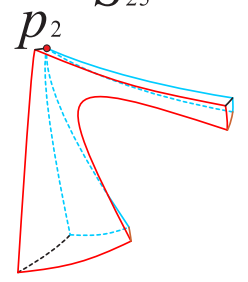

$S_{28}$

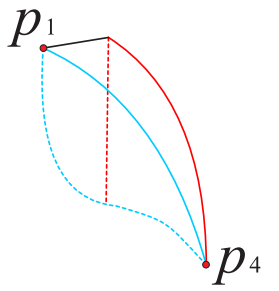

$S_{23}$

Figure 26. The ten subregions inside the region $S_{2}$ of the Poincare ball.

As shown in Figure 25, the top surface of the subregion $S_{11}$ is contained in the Poincare sphere; the bottom surface is contained in the surfaces $h_{1}=0$ and $h_{2}=0$; the bottom plane is contained in the invariant plane $u=0$; the left and right sides are contained in 
the surfaces $f_{-}(x, z, u)=0$ and $h_{1}=0$; the back plane is contained in the invariant plane $z=0$. Noting the dynamic behavior of the orbits in Table 9, we find that all the orbits in this subregion increase monotonically, which means that the orbits in this subregion start at the finite equilibrium points $p_{1}, p_{2}$ and $p_{4}$, or come from the subregion $S_{12}$, eventually approaching the infinite equilibrium points on the Poincaré sphere.

Table 9. Dynamical behavior in the nineteen subregions.

\begin{tabular}{cll}
\hline Subregions & Corresponding Region & Increase or Decrease \\
\hline$S_{11}$ & $h_{1}>0, h_{2}>0, h_{3}>0, z>0, u>0$ & $\dot{x}>0, \dot{z}>0, \dot{u}>0$ \\
\hline$S_{12}$ & $h_{1}>0, h_{2}<0, h_{3}>0, z>0, u>0$ & $\dot{x}>0, \dot{z}<0, \dot{u}>0$ \\
\hline$S_{13}$ & $h_{1}<0, h_{2}>0, h_{3}>0, z>0, u>0$ & $\dot{x}<0, \dot{z}>0, \dot{u}>0$ \\
\hline$S_{14}$ & $h_{1}<0, h_{2}<0, h_{3}>0, z>0, u>0$ & $\dot{x}<0, \dot{z}<0, \dot{u}>0$ \\
\hline$S_{15}$ & $h_{1}<0, h_{2}<0, h_{3}<0, z>0, u>0$ & $\dot{x}<0, \dot{z}<0, \dot{u}<0$ \\
\hline$S_{16}$ & $h_{1}>0, h_{2}<0, h_{3}<0, z>0, u>0$ & $\dot{x}>0, \dot{z}<0, \dot{u}<0$ \\
\hline$S_{17}$ & $h_{1}>0, h_{2}<0, h_{3}<0, z>0, u>0$ & $\dot{x}>0, \dot{z}<0, \dot{u}<0$ \\
\hline$S_{18}$ & $h_{1}>0, h_{2}<0, h_{3}>0, z>0, u>0$ & $\dot{x}>0, \dot{z}<0, \dot{u}>0$ \\
\hline$S_{19}$ & $h_{1}<0, h_{2}>0, h_{3}>0, z>0, u>0$ & $\dot{x}<0, \dot{z}>0, \dot{u}>0$ \\
\hline$S_{21}$ & $h_{1}>0, h_{2}>0, h_{3}>0, z>0, u<0$ & $\dot{x}>0, \dot{z}>0, \dot{u}<0$ \\
\hline$S_{22}$ & $h_{1}>0, h_{2}<0, h_{3}>0, z>0, u<0$ & $\dot{x}>0, \dot{z}<0, \dot{u}<0$ \\
\hline$S_{23}$ & $h_{1}<0, h_{2}>0, h_{3}>0, z>0, u<0$ & $\dot{x}<0, \dot{z}>0, \dot{u}<0$ \\
\hline$S_{24}$ & $h_{1}<0, h_{2}<0, h_{3}>0, z>0, u<0$ & $\dot{x}<0, \dot{z}<0, \dot{u}<0$ \\
\hline$S_{25}$ & $h_{1}<0, h_{2}<0, h_{3}<0, z>0, u<0$ & $\dot{x}<0, \dot{z}<0, \dot{u}>0$ \\
\hline$S_{26}$ & $h_{1}>0, h_{2}<0, h_{3}<0, z>0, u<0$ & $\dot{x}>0, \dot{z}<0, \dot{u}>0$ \\
\hline$S_{27}$ & $h_{1}>0, h_{2}<0, h_{3}>0, z>0, u<0$ & $\dot{x}>0, \dot{z}<0, \dot{u}<0$ \\
\hline$S_{28}$ & $h_{1}>0, h_{2}>0, h_{3}>0, z>0, u<0$ & $\dot{x}>0, \dot{z}>0, \dot{u}<0$ \\
\hline$S_{29}$ & $h_{1}<0, h_{2}>0, h_{3}>0, z>0, u<0$ & $\dot{x}<0, \dot{z}>0, \dot{u}<0$ \\
\hline$S_{210}$ & $h_{1}<0, h_{2}>0, h_{3}>0, z>0, u<0$ & $\dot{x}<0, \dot{z}>0, \dot{u}<0$ \\
\hline
\end{tabular}

For the subregion $S_{12}$, the left and right surfaces are contained in the surfaces $h_{2}=0$ and $h_{1}=0$, respectively; the bottom plane is contained in the invariant plane $u=0$; and the front plane is contained in the Poincare sphere. Table 9 shows that the orbits in this subregion increase monotonically along the positive direction of the $x$-axis and $u$-axis, and decrease monotonically along the positive direction of the $z$-axis. Therefore, the orbits in this subregion start from the infinite equilibrium points on the Poincare sphere, and then cross the left surface $h_{2}=0$ into the subregion $S_{11}$.

In the subregion $S_{13}$ the left and right surfaces are contained in the surfaces $h_{1}=0$ and $h_{2}=0$, respectively; the bottom plane is contained in the invariant plane $u=0$; the back plane is contained in the invariant plane $z=0$; and the front plane is contained in the Poincare sphere. Table 9 means that the orbits in this subregion decrease monotonically along the positive direction of the $x$-axis, while the orbits increase monotonically along the other two axes. It means that the orbits in this subregion start at the finite equilibrium point $p_{1}$ and cross the surface $h_{2}=0$ into the neighboring subregion $S_{14}$.

In the subregion $S_{14}$ the left side surface is contained in the surfaces $h_{1}=0$ and $h_{2}=0$, the right side surface is contained in the surface $h_{3}=0$, the back plane is contained in the invariant plane $z=0$, the back surface is contained in the surface $h_{1}=0$, the bottom plane is contained in the invariant plane $u=0$ and the front surface is contained in the Poincaré sphere. From Table 9 we know that the orbits in this subregion are monotonically decreasing along the positive direction of the $x$-axis and $z$-axis, while the orbits are monotonically increasing along the $u$-axis in the positive direction. Then, the orbits start at the finite equilibrium point $p_{4}$, or the equilibrium points on the Poincare sphere at infinity in this 
subregion; cross the surfaces $h_{1}=0$ and $h_{3}=0$; and finally, enter the adjacent subregions $S_{15}$ and $S_{18}$, respectively.

The subregion $S_{15}$ is composed of the left side surface contained in the surface $h_{3}=0$, the right side surface contained in the surface $h_{1}=0$, the bottom surface contained in the invariant plane $u=0$ and the front surface contained in the Poincaré sphere. From Table 9 we find that the orbits are monotonically decreasing along the three directions in the positive direction in this subregion, so the orbits originate from the subregion $S_{14}$ or from the infinite equilibrium points on the Poincare sphere in the subregion $S_{15}$, and then cross the surface $h_{1}=0$ into the connecting subregion $S_{16}$.

The top surface of the subregion $S_{16}$ is contained in the surface $h_{3}=0$, the front left surface is contained in the surface $h_{1}=0$, the front right surface is contained in the Poincare sphere and the bottom plane is contained in the invariant plane $u=0$. The subregion $S_{17}$ is composed of the front surface contained in $h_{3}=0$ and the back plane contained in the invariant plane $z=0$. Table 9 indicates that the orbits have the same dynamic behavior in these two subregions, they increase monotonically along the positive direction of the $x$-axis and decrease monotonically along the other two axes, so the orbits in the subregion $S_{16}$ start from the subregion $S_{15}$ or from the infinite equilibrium points on Poincare sphere, then cross the surface $h_{3}=0$ into the subregion $S_{18}$. The orbits in the subregion $S_{17}$ come from the subregion $S_{18}$ and finally return to this subregion, that is the orbits in the subregion $S_{18}$ can cross the subregion $S_{17}$ from the right to left.

The top surface of the subregion $S_{18}$ is contained in the surface $h_{2}=0$; the bottom surface is contained in the surface $h_{3}=0$; the bottom plane is contained in the invariant plane $u=0$; the left side surface is contained in the surface $h_{1}=0$; the front surface is contained in the Poincare sphere; the back surface is contained in the invariant plane $z=0$ and the surface $h_{3}=0$. Table 9 implies that the orbits decrease monotonically along the $z$-axis and increase monotonically along the other two axes in this subregion, so some orbits may start from infinite equilibrium points on Poincaré sphere, some orbits start from the finite equilibrium point $p_{2}$, and then cross the surface $h_{2}=0$ into the subregion $S_{18}$ and eventually into the subregion $S_{11}$.

The front surface of the subregion $S_{19}$ is contained in the surface $h_{1}=0$; the bottom plane is contained in the invariant plane $u=0$; the back surface part is contained in the invariant plane $z=0$ and the surface $f_{-}(x, z, u)=0$. Table 9 shows that the orbits in $S_{19}$ decrease monotonically along the positive direction of the $x$-axis, and increase monotonically along the positive direction of the other two axes, so the orbits in this subregion start at the finite equilibrium point $p_{2}$ and then tend to equilibrium points at infinity on the Poincare sphere or go through the right part of the surface $f_{-}(x, z, u)=0$ into outer space. Therefore, the dynamic behavior of the orbits of system (7) in the region $S_{1}$ can be concluded into the following flow chart.

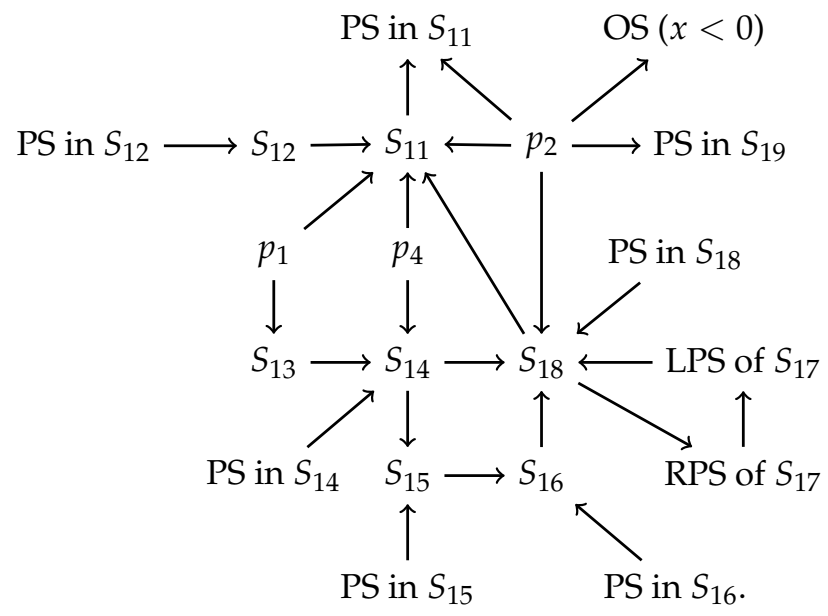


Note: OS represents the outer space.

The above flow chart in the region $S_{1}$ indicates that the orbits of system (7) have an $\alpha$-limit at the finite equilibrium points $p_{1}, p_{2}$ and $p_{4}$; and on the Poincaré sphere restricted to the subregions $S_{12}, S_{14}, S_{15}, S_{16}$ and $S_{18}$. In addition the orbits have an $\omega$-limit in the subregions $S_{11}$ and $S_{19}$, which are restricted to Poincaré sphere. Here $\alpha$-limit and $\omega$-limit are terms in dynamic systems, which can be regarded as de Sitter past attractors and de Sitter future attractors, respectively.

For the region $S_{2}$ the left surface of the subregion $S_{21}$ is contained in the surface $f_{-}(x, z, u)=0$; the right surface is contained in the surfaces $h_{1}=0$ and $h_{2}=0$; the top plane is contained in the invariant plane $u=0$; the bottom surface and the front triangleshaped surface are contained in the Poincare sphere; and the back plane is contained in the invariant plane $z=0$. Table 9 shows that the orbits in this subregion decrease monotonically along the positive direction of $u$-axis, and monotonically increase along the positive directions of the other two axes, so the orbits in this subregion start at finite equilibrium points $p_{1}$ and $p_{4}$, or from the neighboring subregion $S_{22}$, they eventually tend to the infinite equilibrium points of the Poincare sphere that is restricted to the subregion $S_{21}$ or go through the left part of the surface $f_{-}(x, z, u)=0$ into the outer space.

The top plane and surface of the subregion $S_{22}$ are contained in the invariant plane $u=0$ and the surface $h_{1}=0$, respectively; the back plane is contained in the invariant plane $z=0$; the left side surface is contained in the surfaces $h_{2}=0$ and $f_{-}(x, z, u)=0$; the right side surface is contained in the surface $h_{3}=0$; and the front and bottom surfaces are contained in the Poincare sphere. Table 9 implies that the orbits in this subregion increase monotonically along the positive $x$-axis direction and decrease monotonically along the positive $z$-axis and $u$-axis directions, which means that these orbits may come from the subregions $S_{24}$ and $S_{26}$, and then enter in the subregion $S_{21}$ or tend to the infinite equilibrium points on the Poincaré sphere in the subregion $S_{22}$, or cross the surface $f_{-}(x, z, u)=0$ into the outer space.

The structure of subregions $S_{23}$ and $S_{24}$ and the dynamic behavior of the orbits in them are the same as those of subregions $R_{23}$ and $R_{24}$ (see Figures 23 and 26). Thus the orbits in the subregion $S_{23}$ start at the finite equilibrium point $p_{1}$ and cross the surface $h_{2}=0$, and then enter into the subregion $S_{24}$; and the orbits in the subregion $S_{24}$ start at the finite equilibrium point $p_{4}$, the connecting subregion $S_{23}$. The infinite equilibrium points start on the Poincare sphere, and then cross the surfaces $h_{1}=0$ and $h_{3}=0$, and eventually tend to the subregions $S_{22}$ and $S_{25}$, respectively.

For the subregion $S_{25}$ except that the surface on the lower left side is contained in the surface $f_{-}(x, z, u)=0$, the remaining structure is the same as that of $R_{25}$. According to the Tables 6 and 9 the monotonicity of the orbits in these two subregions are also the same, so the orbits in the subregion $S_{25}$ start from the infinity equilibrium points on the Poincaré sphere, or from the outer space through the left side surface $f_{-}(x, z, u)=0$, but all eventually cross the surface $h_{1}=0$ into the subregion $S_{26}$.

The top plane of the subregion $S_{26}$ is contained in the invariant plane $u=0$; the left and right side surfaces are contained in the surfaces $h_{3}=0$ and $f_{-}(x, z, u)=0$; and the front surface located at the upper left corner is contained in the surface $h_{1}=0$. The front surface containing the infinite equilibrium point $e_{4}$ is contained in the Poincare sphere. Table 9 shows that the orbits decrease monotonically along the positive direction of the $z$-axis in the subregion $S_{26}$, while the orbits along the positive direction of the remaining two axes is just the opposite. Therefore, the orbits in this subregion start at the infinite equilibrium points on the Poincare sphere or from the subregion $S_{27}$ and the outer space passing through the right side surface $f_{-}(x, z, u)=0$, and finally enter the subregion $S_{22}$ through the left side surface $h_{3}=0$.

The front and back surfaces of the subregion $S_{27}$ are contained in the surfaces $h_{3}=0$ and $h_{2}=0$, respectively; the back plane is contained in the invariant plane $z=0$; the top plane is contained in the invariant plane $u=0$; and the right side surface is contained in the Poincaré sphere and the right part of the surface $f_{-}(x, z, u)=0$. Table 9 shows that 
the orbits decrease monotonically along the positive direction of the $x$-axis and increase monotonically along the positive direction of the $z$-axis and $u$-axis in this subregion. In this way the orbits in $S_{27}$ start from the adjacent subregion $S_{28}$ or come from the outer space passing through the right side surface $f_{-}(x, z, u)=0$ and the infinite equilibrium points on the Poincaré sphere, and finally pass through the front surface $h_{3}=0$ and enter the subregion $S_{26}$.

The structure of the subregion $S_{28}$ is similar to the subregion $S_{27}$ discussed above, except that the back surface of the subregion $S_{27}$ happens to be the front surface of the subregion $S_{28}$, and the back surface of the subregion $S_{28}$ is contained in the surface $h_{1}=0$. Note that Table 9 implies that the orbits monotonically decrease along the positive direction of the $u$-axis in this subregion, and increase monotonically along the positive direction of the other two axes. This means that the orbits start at the finite equilibrium point $p_{2}$, and then cross the surface $h_{2}=0$ into the subregion $S_{27}$.

The subregions $S_{29}$ and $S_{210}$ are connected by the finite equilibrium point $p_{2}$. Their front surfaces are contained in the surface $h_{1}=0$; the bottom surface of $S_{29}$ and the right surface of $S_{210}$ are contained in the Poincaré sphere; the right side surface of $S_{29}$ and the back surface of $S_{210}$ are contained in the right part of the surface $f_{-}(x, z, u)=0$; and the back surface plane of $S_{29}$ is contained in the invariant plane $z=0$. From Table 9 we find that the dynamic behavior of the orbits in these two subregions is the same, both decrease monotonically along the positive direction of the $x$-axis and $u$-axis, and increase monotonically along the positive direction of the $z$-axis. Thus, the orbits in these two subregions start at the finite equilibrium point $p_{2}$, and then tend to the Poincare sphere or go through the surface $f_{-}(x, z, u)=0$ into the outer space.

Accordingly the dynamic behavior of system (7) in the region $S_{2}$ can be shown by the following flow chart.

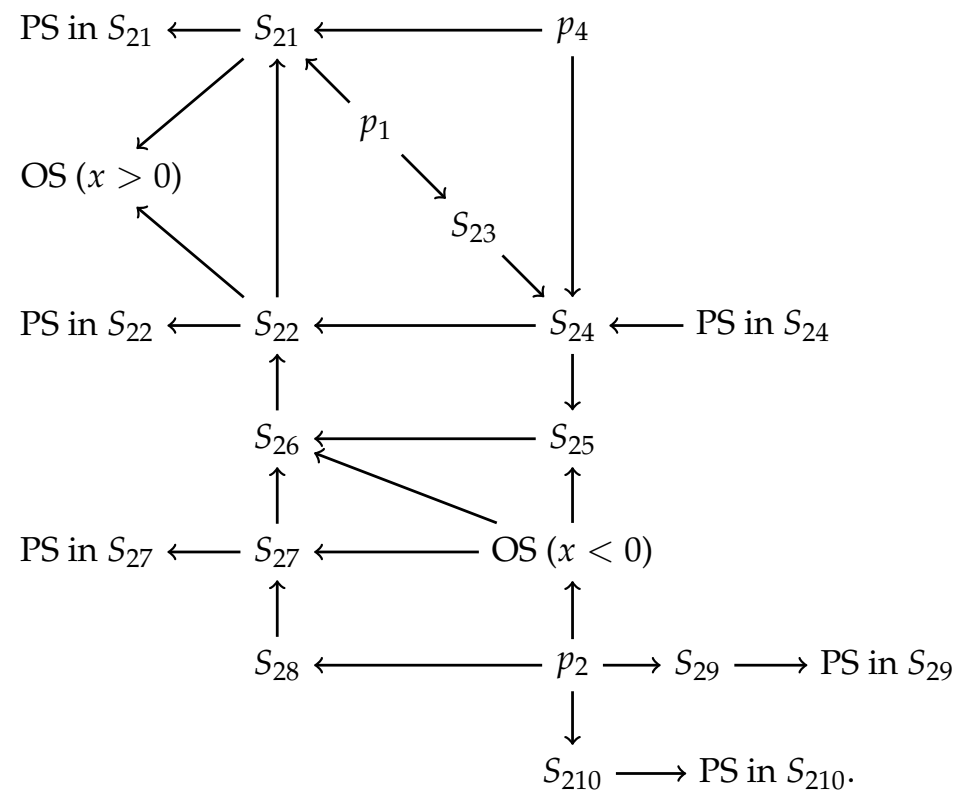

This flow chart in the region $S_{2}$ implies that the orbits of system (7) have an $\alpha$-limit at the finite equilibrium points $p_{1}, p_{2}$ and $p_{4}$; and at the infinite equilibrium points on the Poincare sphere restricted to the subregion $S_{24}$. Besides. the orbits have an $\omega$-limit in the subregions $S_{21}, S_{22}, S_{27}, S_{29}$ and $S_{210}$, which are restricted to Poincaré sphere. 
When the field potential $V(\phi)$ takes the form of constant, i.e. $s=0$. Then system $(7)$ is reduced to

$$
\begin{aligned}
& \frac{d x}{d t}=x\left[3 x^{2}+2(u-z) z-3\right], \\
& \frac{d z}{d t}=z\left[3 x^{2}+2(u-z) z-2\right] \\
& \frac{d u}{d t}=u\left[3 x^{2}+2(u-z) z\right] .
\end{aligned}
$$

Now we continue to describe the global dynamics of system (31) in the closed and open universe in Sections 5 and 6, respectively.

5. Case III: $s=0, k=1$

\subsection{Phase Portraits on the Invariant Planes and Surface}

In this section, we will investigate the local and global phase portraits of the finite and infinite equilibrium points of system (31). The phase portraits on the invariant planes $x=0, z=0$ and $u=0$, and on the invariant surface $f_{+}(x, z, u)=0$ are studied in what follows.

5.1.1. The Invariant Plane $x=0$

On this plane system (31) is

$$
\frac{d z}{d t}=2 z\left(u z-z^{2}-1\right), \quad \frac{d u}{d t}=2 u z(u-z) .
$$

It is easy to check that this system has no other finite equilibrium points except the straight line $z=0$ which is full of the equilibrium points of system (32).

By using the Poincaré compactification $z=1 / V$ and $u=U / V$, we obtain that system (32) on the local chart $U_{1}$ has the form

$$
\frac{d U}{d t}=2 U V^{2}, \quad \frac{d V}{d t}=2 V\left(1-U+V^{2}\right) .
$$

Rescaling the time via $d \tau_{8}=2 V d t$ the previous system becomes

$$
\frac{d U}{d \tau_{8}}=U V, \quad \frac{d V}{d \tau_{8}}=1-U+V^{2}
$$

It admits an infinite equilibrium point $e_{11}=(1,0)$ with eigenvalues $\pm i$, which indicates that $e_{11}$ may be either a center or a weak focus of this system. Note that $\left(1-2 U+V^{2}\right) / U^{2}=C$ is a first integral of system (34). Thus $e_{11}$ is a center.

On the local chart $U_{2}$ we have $z=U / V$ and $u=1 / V$. Thus, system (32) can be rewritten as

$$
\frac{d U}{d t}=-2 U V^{2}, \quad \frac{d V}{d t}=2(U-1) U V
$$

On the local chart $U_{2}$ we only need to examine the origin $(0,0)$ of system $(35) . \mathrm{Ob}-$ viously $e_{12}$ is an equilibrium point with eigenvalues zero, the conventional eigenvalue method cannot be used to determine the type of $e_{12}$ and its local phase portrait. We apply horizontal blow-up by introducing the transformation $W=U / V$ (see [57] for more details), and then we obtain

$$
\frac{d V}{d t}=2 V^{2} W(V W-1), \quad \frac{d W}{d t}=-2 V W\left(V-W+V W^{2}\right) .
$$


Doing the time transformation $d \tau_{9}=2 V W d t$ we eliminate the common factor, and we have

$$
\frac{d V}{d \tau_{9}}=V(V W-1), \quad \frac{d W}{d \tau_{9}}=-V+W-V W^{2} .
$$

Then the equilibrium point $e_{12}=(0,0)$ of system (37) with eigenvalues \pm 1 is a hyperbolic saddle.

Eliminating the common factor $2 U V$ of system (35) by taking $d \tau_{10}=2 U V d t$ yields $d U / d \tau_{10}=-V$ and $d V / d \tau_{10}=U-1$, which implies that the orbits of the local phase portrait of the infinite equilibrium point $e_{12}$ decreases monotonically along the $V$-axis, and increases monotonically along the $U$-axis when $V<0$, or decreases monotonically along the $U$-axis when $V>0$. Therefore, the local phase portrait of $e_{12}$ and the global phase portrait of system (32) are shown in Figure 27.

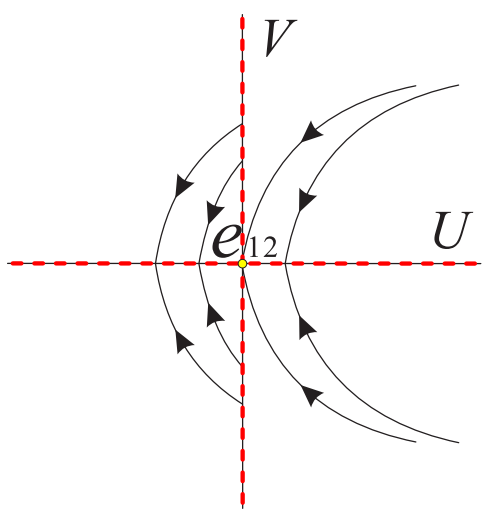

(a)

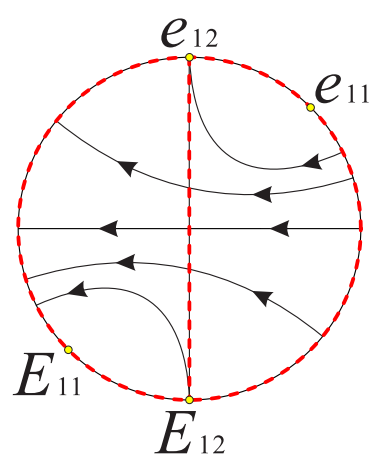

(b)

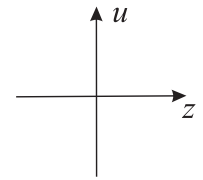

Figure 27. (a) The local phase portrait at the infinite equilibrium point $e_{12}$. (b) The global phase
portrait of system (32). $E_{11}$ and $E_{12}$ are the diametrally opposite equilibrium points of $e_{11}$ and $e_{12}$ in the Poincaré disc, respectively.

5.1.2. The Invariant Plane $z=0$

On this plane system (31) becomes

$$
\frac{d x}{d t}=3 x\left(x^{2}-1\right), \quad \frac{d u}{d t}=3 u x^{2} .
$$

This system is exactly the same as system (9) in [15], so the global phase portrait of system (38) is shown in Figure 28. The finite equilibrium points $e_{13}=(1,0)$ and $e_{14}=(-1,0)$ are hyperbolic unstable nodes. Besides the line $x=0$ and the infinity of the local chart $U_{1}$ are filled with equilibrium points (see [15] for more details).

5.1.3. The Invariant Plane $u=0$

On this plane system (31) is reduced to

$$
\frac{d x}{d t}=x\left(3 x^{2}-2 z^{2}-3\right), \quad \frac{d z}{d t}=z\left(3 x^{2}-2 z^{2}-2\right) .
$$

Note that system (39) is the same as system (9) in [15], so the global phase portrait of system (39) is illustrated in Figure 29. The finite equilibrium point $e_{16}=(0,0)$ is a hyperbolic stable node, $e_{17}=(1,0)$ and $e_{18}=(-1,0)$ are hyperbolic unstable nodes. In addition the infinity of system (39) is full of the equilibrium points (see [15] for more details). 


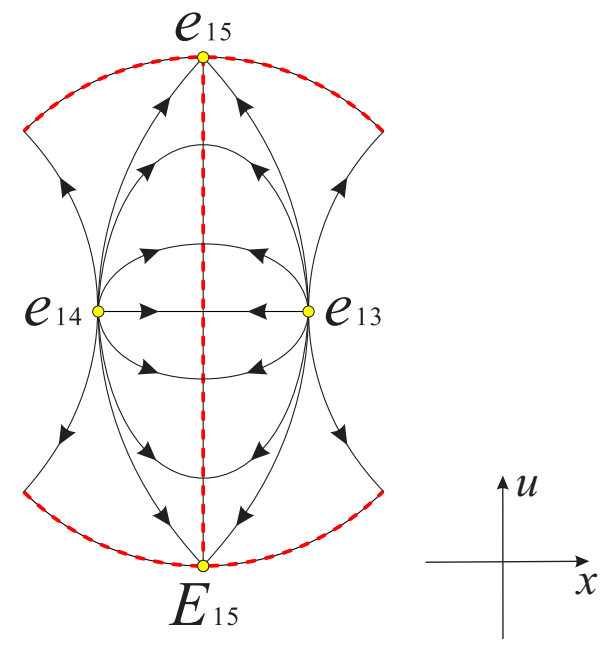

Figure 28. The global phase portrait of system (38) on the invariant plane $z=0$ restricted to the region $x^{2}-u^{2} \leq 1$. $E_{15}$ is the diametrically opposite point of $e_{15}$ on Poincaré disc.

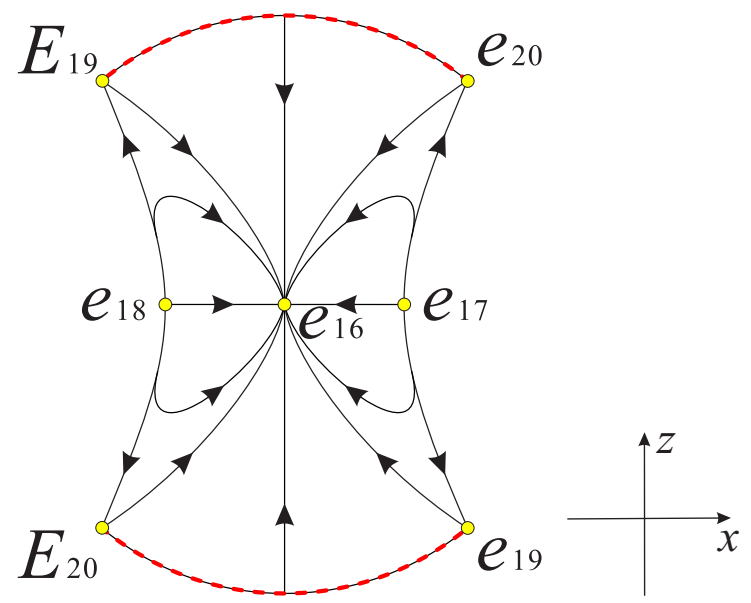

Figure 29. The global phase portrait of system (39) on the invariant plane $u=0$ restricted to the region $x^{2}-z^{2} \leq 1$. $E_{19}$ and $E_{20}$ are the diametrically opposite points of $e_{19}$ and $e_{20}$ on Poincaré disc, respectively.

5.1.4. The Invariant Surface $f_{+}(x, z, u)=0$

On this surface system (31) is rewritten in the same form as systems (16) and (17) in Section 3.1.3, so the global phase portrait on the invariant surface $f_{+}(x, z, u)=0$ can be found in Figures 9 and 10, respectively.

\subsubsection{The Finite Equilibrium Points}

It is easy to find that there are two finite equilibrium points $q_{1}=(1,0,0)$ and $q_{2}=$ $(-1,0,0)$ of system $(31), q_{1}$ and $q_{2}$ with the same eigenvalues 6,3 and 1 are hyperbolic unstable nodes. Besides $u$-axis is full of equilibrium points of system (31).

\subsubsection{Phase Portrait on the Poincaré Sphere at Infinity}

According to the three-dimensional Poincaré compactification, system (31) can be rewritten as follows. On the local chart $U_{1}$

$$
\frac{d z_{1}}{d t}=z_{1} z_{3}^{2}, \frac{d z_{2}}{d t}=3 z_{2} z_{3}^{2}, \frac{d z_{3}}{d t}=z_{3}\left(2 z_{1}^{2}-2 z_{1} z_{2}+3 z_{3}^{2}-3\right) .
$$


On the local chart $U_{2}$

$$
\frac{d z_{1}}{d t}=-z_{1} z_{3}^{2}, \frac{d z_{2}}{d t}=2 z_{2} z_{3}^{2}, \frac{d z_{3}}{d t}=z_{3}\left(2-3 z_{1}^{2}-2 z_{2}+2 z_{3}^{2}\right) .
$$

On the local chart $U_{3}$

$$
\frac{d z_{1}}{d t}=-3 z_{1} z_{3}^{2}, \frac{d z_{2}}{d t}=-2 z_{2} z_{3}^{2}, \frac{d z_{3}}{d t}=-3 z_{1}^{2} z_{3}+2\left(z_{2}-1\right) z_{2} z_{3} .
$$

It is noted that Equations (40)-(42) are the same as systems (23), (27) and (29) when $s=0$ in Section 3.1.5, respectively, so the Poincaré sphere is filled with equilibrium points of system (31) at infinity.

\subsection{Phase Portrait Inside the Poincaré Ball Restricted to the Physical Region of Interest $x^{2}-(u-z)^{2} \leq 1$}

As mentioned in Section 2 system (31) is invariant under the three symmetries $(x, z, u) \mapsto(-x,-z,-u),(x, z, u) \mapsto(x,-z,-u)$ and $(x, z, u) \mapsto(-x, z, u)$. Here we divide the Poincaré ball into four regions as follows:

$$
\begin{aligned}
& Q_{1}: x \geq 0, z \geq 0 . \quad Q_{2}: x \leq 0, z \geq 0 . \\
& Q_{3}: x \leq 0, z \leq 0 . \quad Q_{4}: x \geq 0, z \leq 0 .
\end{aligned}
$$

Due to the above symmetries with respect to the origin, the $x$-axis and the invariant plane $x=0$, we only need to discuss the phase portrait of system (31) in the region $Q_{1}$ restricted to the region $x^{2}-(u-z)^{2} \leq 1$.

By joining the phase portraits on the invariant planes $x=0, z=0$ and $u=0$; on the invariant surface $f_{+}(x, z, u)=0$; and on the Poincare sphere at infinity, the phase portrait on the boundary of the region $Q_{1}$ is displayed in Figure 30. It is noted that all the equilibrium points on the $u$-axis are stable along the two intersecting boundary planes $x=0$ and $z=0$, and the finite equilibrium point $q_{1}$ is unstable on the invariant boundary plane $z=0$ and on the invariant boundary surface $f_{+}(x, z, u)=0$.

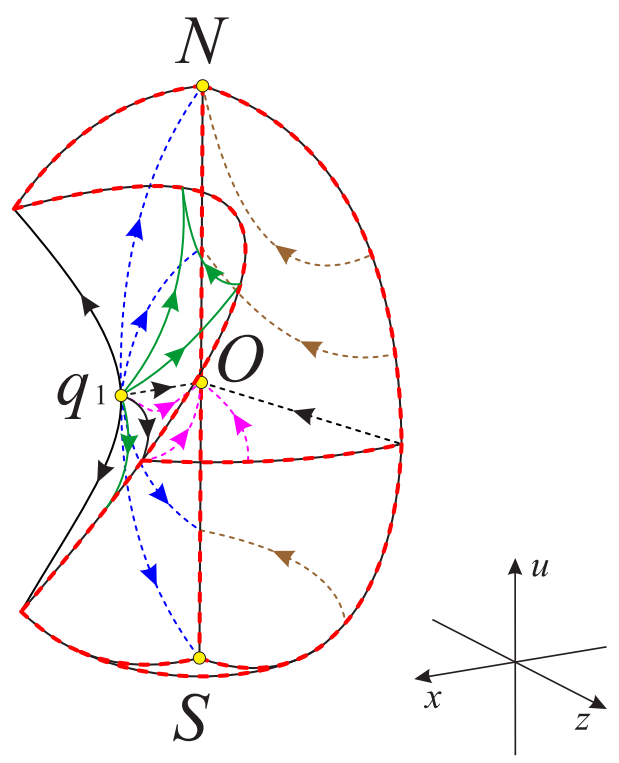

Figure 30. Phase portrait on the boundary of the region $Q_{1}$, and $O$ denotes the center of the Poincaré ball. 


\subsection{Dynamics in the Interior of the Region $Q_{1}$}

The dynamics of system (31) inside the region $Q_{1}$ is governed by the behavior of the orbits in the planes and surfaces as follows:

$$
\begin{aligned}
& x=0, z=0, u=0, f_{+}(x, z, u)=0, \\
& h_{0}(x, z, u)=0, h_{2}(x, z, u)=0, h_{3}(x, z, u)=0,
\end{aligned}
$$

where $h_{0}(x, z, u)=h_{2}(x, z, u)-1=h_{3}(x, z, u)-3=3 x^{2}+2(u-z) z-3$. Then the interior of region $Q_{1}$ is separated into ten subregions $Q_{1 n}, n=(1, \ldots, 10)$ by the above planes and surfaces, from these there are six subregions above the invariant plane $u=0$ and four subregions below. See Figures 31 and 32 for more details. The signs of the functions $h_{0}, h_{2}$ and $h_{3}$ in the subregion of $Q_{1}$ are given in Table 10.
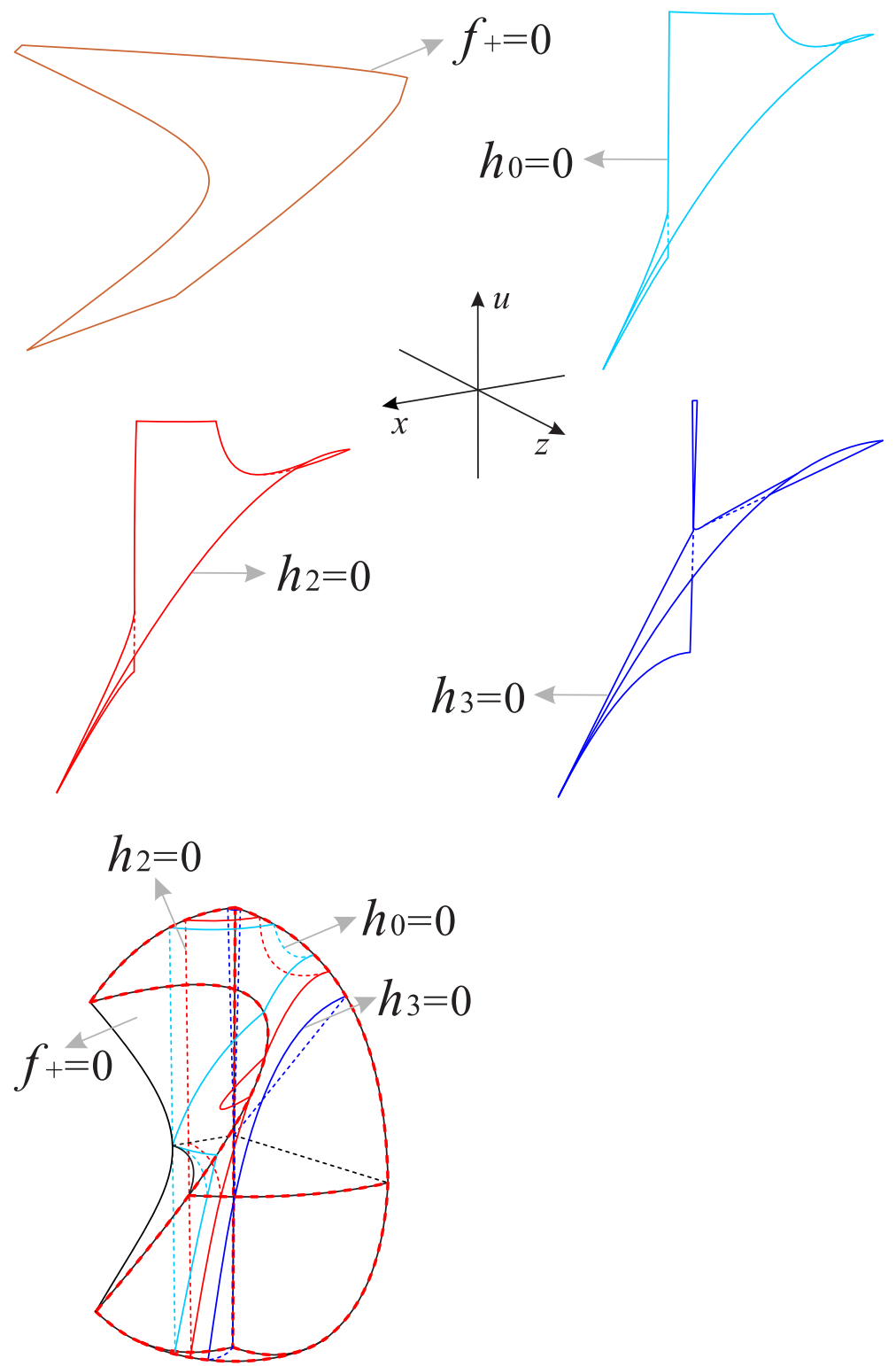

Figure 31. The surfaces $h_{0}, h_{2}$ and $h_{3}$ restricted to the surface $f_{+}(x, z, u)=0$ and the region $Q_{1}$ of the Poincaré ball. 

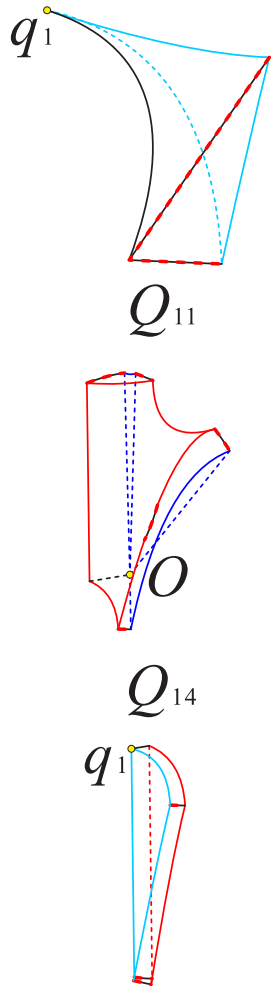

$Q_{18}$

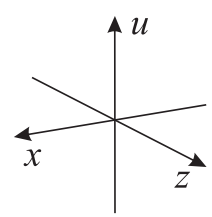

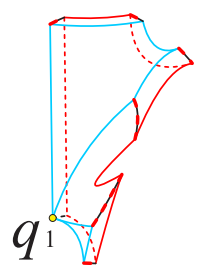

$Q_{12}$

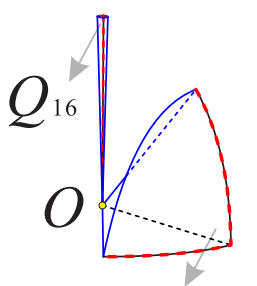

$Q_{15}$

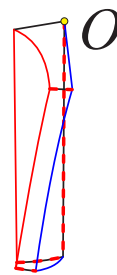

$Q_{19}$

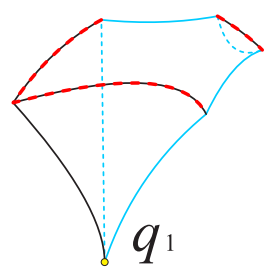

$Q_{13}$

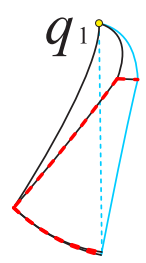

$Q_{17}$

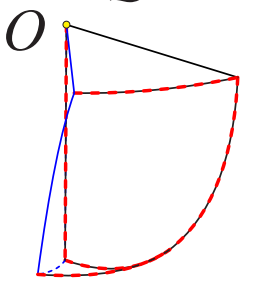

$Q_{110}$

Figure 32. The ten subregions inside the region $Q_{1}$ of the Poincare ball.

Table 10. Signs of functions $h_{0}, h_{2}$ and $h_{3}$ in the subregion of $Q_{1}$.

\begin{tabular}{cll}
\hline Functions & Positive & Negative \\
\hline$h_{0}$ & $Q_{11}, Q_{13}, Q_{17}$ & $Q_{12}, Q_{14}, Q_{15}, Q_{16}, Q_{18}, Q_{19}, Q_{110}$ \\
\hline$h_{2}$ & $Q_{11}, Q_{12}, Q_{13}, Q_{17}, Q_{18}$ & $Q_{14}, Q_{15}, Q_{16}, Q_{19}, Q_{110}$ \\
\hline$h_{3}$ & $Q_{11}, Q_{12}, Q_{13}, Q_{14}, Q_{17}, Q_{18}, Q_{19}$ & $Q_{15}, Q_{16}, Q_{110}$ \\
\hline
\end{tabular}

According to Figure 32, it can be seen that the bottom plane of the subregion $Q_{11}$ is contained in the invariant plane $u=0$ the left surface is contained in the invariant surface $f_{+}(x, z, u)=0$, the right surface is contained in the surface $h_{1}=0$ and the front surface is contained in the Poincare sphere of the subregion. Table 11 shows that the orbits of system (31) increase monotonically along the positive directions of the three coordinate axes in this subregion, so the orbits in this subregion start at the finite equilibrium point $q_{1}$ and then finally tend to the equilibrium points at infinity of Poincaré sphere.

Similarly, the bottom plane, the back-left plane and the right-back plane of the subregion $Q_{12}$ are contained in the invariant planes $u=0, z=0$ and $x=0$, respectively. The back surface is contained in the surface $h_{2}=0$, the top-back surface and the front quadrilateral surface are contained in the Poincaré sphere, the upper and lower surfaces on the left side are contained in the surface $h_{1}=0$ and in the invariant plane $f_{+}(x, z, u)=0$, respectively. Then the orbits monotonically decrease along the positive direction of the $x$-axis in this subregion, and monotonically increase along the positive direction of the 
other two coordinate axes, that is, the orbits start at the finite equilibrium point $q_{1}$ and then go to the subregions $Q_{13}$ and $Q_{14}$.

Table 11. Dynamical behavior in the ten subregions.

\begin{tabular}{cll}
\hline Subregions & Corresponding Region & Increase or Decrease \\
\hline$Q_{11}$ & $h_{0}>0, h_{2}>0, h_{3}>0, x>0, z>0, u>0$ & $\dot{x}>0, \dot{z}>0, \dot{u}>0$ \\
\hline$Q_{12}$ & $h_{0}<0, h_{2}>0, h_{3}>0, x>0, z>0, u>0$ & $\dot{x}<0, \dot{z}>0, \dot{u}>0$ \\
\hline$Q_{13}$ & $h_{0}>0, h_{2}>0, h_{3}>0, x>0, z>0, u>0$ & $\dot{x}>0, \dot{z}>0, \dot{u}>0$ \\
\hline$Q_{14}$ & $h_{0}<0, h_{2}<0, h_{3}>0, x>0, z>0, u>0$ & $\dot{x}<0, \dot{z}<0, \dot{u}>0$ \\
\hline$Q_{15}$ & $h_{0}<0, h_{2}<0, h_{3}<0, x>0, z>0, u>0$ & $\dot{x}<0, \dot{z}<0, \dot{u}<0$ \\
\hline$Q_{16}$ & $h_{0}<0, h_{2}<0, h_{3}<0, x>0, z>0, u>0$ & $\dot{x}<0, \dot{z}<0, \dot{u}<0$ \\
\hline$Q_{17}$ & $h_{0}>0, h_{2}>0, h_{3}>0, x>0, z>0, u<0$ & $\dot{x}>0, \dot{z}>0, \dot{u}<0$ \\
\hline$Q_{18}$ & $h_{0}<0, h_{2}>0, h_{3}>0, x>0, z>0, u<0$ & $\dot{x}<0, \dot{z}>0, \dot{u}<0$ \\
\hline$Q_{19}$ & $h_{0}<0, h_{2}<0, h_{3}>0, x>0, z>0, u<0$ & $\dot{x}<0, \dot{z}<0, \dot{u}<0$ \\
\hline$Q_{110}$ & $h_{0}<0, h_{2}<0, h_{3}<0, x>0, z>0, u<0$ & $\dot{x}<0, \dot{z}<0, \dot{u}>0$ \\
\hline
\end{tabular}

The front surface of the subregion $Q_{13}$ is contained in the invariant plane $f_{+}(x, z, u)=$ 0 ; the top surface is contained in the Poincare sphere; the left and right planes of the back are contained in the invariant planes $z=0$ and $x=0$, respectively; and the middle surface of the back is contained in the surface $h_{1}=0$. Table 11 shows that the orbits in this subregion increase monotonically along the positive direction of the three coordinate axes, this means that the orbits in this subregion start from the finite equilibrium point $q_{1}$, and may also come from the adjacent subregion $Q_{12}$. They tend to the Poincaré sphere at infinity.

The left and right planes of the back of the subregion $Q_{14}$ are contained in the invariant planes $z=0$ and $x=0$, respectively; the back surface is contained in the surface $h_{3}=0$; the top and front surfaces are contained in the Poincaré sphere; the left surface is contained in the surface $h_{2}=0$ and in the invariant surface $f_{+}(x, z, u)=0$; the right side surface is contained in the surface $h_{3}=0$; and the bottom plane is contained in the invariant plane $u=0$. Note Table 11 states that the orbits in this subregion decrease monotonically along the positive direction of the $x$-axis and $z$-axis, and increase monotonically along the positive direction of the $u$-axis, so the orbits in this subregion start from the infinite equilibrium points in the Poincare sphere or in the subregion $Q_{12}$, and then go to the subregion $Q_{16}$ covering the $u$-axis, because the entire $u$-axis is filled with the equilibrium points of system (31).

The left side surface of the subregion $Q_{15}$ is contained in the surface $h_{3}=0$; the back plane and the bottom plane are contained in the invariant planes $x=0$ and $u=0$, respectively; and the front surface is contained in the Poincaré sphere. The front surface of the subregion $Q_{16}$ is contained in the surface $h_{3}=0$; the left and right planes of the back are contained in the invariant planes $z=0$ and $x=0$, respectively; and the section line of the invariant planes $z=0$ and $x=0$ in this subregion is the $u$-axis. The subregions $Q_{15}$ and $Q_{16}$ are connected together through the origin of system (31). According to Table 11 the orbits in the two subregions decrease monotonically along the positive directions of the three coordinate axes, indicating that the orbits in the subregion $Q_{15}$ start in the Poincare sphere at infinity and after enter in the subregion $Q_{14}$ or tend to the origin $O$. The orbits in the subregion $Q_{16}$ come from the subregion $Q_{14}$, and finally go to the finite and infinite equilibrium points of the $u$-axis.

The right and left surfaces of the subregion $Q_{17}$ are contained in the surface $h_{1}=0$ and in the invariant surface $f_{+}(x, z, u)=0$; the top plane is contained in the invariant plane $u=0$; the front and bottom surfaces are contained in the Poincare sphere; and the back plane is contained in the invariant plane $z=0$. Table 11 shows that the orbits in this subregion increase monotonically along the positive direction of the $x$-axis and $z$-axis, 
and decrease monotonically along the positive direction of the $u$-axis, which indicate that the orbits actually originate from the finite equilibrium point $q_{1}$ and then run towards the equilibrium points at infinity on the Poincaré sphere.

The subregion $Q_{18}$ has the same composition as in $Q_{17}$ except that the left and right surfaces are contained in the surfaces $h_{1}=0$ and $h_{2}=0$, respectively. We find from Table 11 that the orbits monotonically increase along the positive direction of the $z$-axis and decrease monotonically along the positive directions of the other two coordinate axes. The orbits start at the finite equilibrium point $q_{1}$ in this subregion and finally enter in the subregion $Q_{19}$.

The subregion $Q_{19}$ also has the same composition as $Q_{17}$ except that the left and right surfaces are contained in the surfaces $h_{2}=0$ and $h_{3}=0$, respectively. Table 11 shows that the orbits monotonically decrease along the positive directions of the three coordinate axes. The orbits begin at the infinite equilibrium points on the Poincare sphere or come from the subregion $Q_{18}$, and then tend to the equilibrium points on the $u$-axis.

In the subregion $Q_{110}$ the left and right surfaces are contained in the surface $h_{3}=0$ and in the invariant plane $x=0$, the top plane is contained in the invariant plane $u=0$ and the front and bottom surfaces are contained in the Poincare sphere. It is noted from Table 11 that the orbits in this subregion decrease monotonically along the positive direction of the $x$-axis and $z$-axis, and increase monotonically along the positive direction of the $u$-axis, indicating that the orbits actually start at the equilibrium points on the Poincare sphere at infinity, and eventually tend to the equilibrium points on the $u$-axis.

In summary the dynamics of the orbits in the ten subregions inside the region $Q_{1}$ studied above can be sketched in the following flow chart.

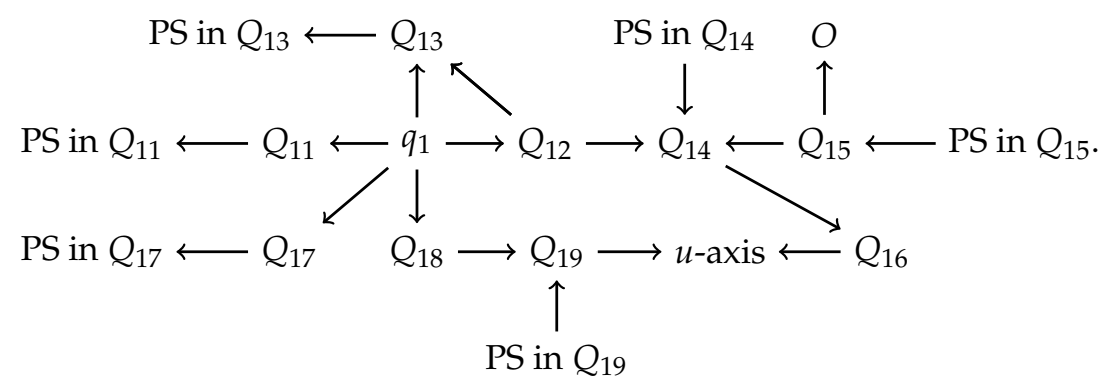

The above flow chart indicates that the orbits of system (31) contained in the region $Q_{1}$ admit an $\alpha$-limit at the finite equilibrium point $q_{1}$. Moreover, the orbits also have an $\alpha$-limit at the equilibrium points in the subregions $Q_{14}, Q_{15}$ and $Q_{19}$ when they are restricted to the Poincaré sphere at infinity. In addition the orbits have an $\omega$-limit at the equilibrium points located on the $u$-axis and at the infinite equilibrium points restricted to the subregions $Q_{11}$, $Q_{13}$ and $Q_{17}$ on the Poincaré sphere.

6. Case IV: $s=0, k=-1$

In this case system (31) has the same phase portraits on the three invariant planes $x=0, z=0$ and $u=0$ as in Sections 5.1.1-5.1.3. In addition the local phase portraits of the finite and infinite equilibrium points on the Poincare sphere are consistent in Sections 5.1.4 and 5.1.5, respectively. However, the physical region of interest $f_{-}(x, z, u)=0$ is no more an invariant surface.

Here we again divide the Poincaré ball into four regions restricted to the region $x^{2}-(u+z)^{2} \leq 1$ as follows:

$$
\begin{array}{ll}
T_{1}: x \geq 0, z \geq 0 . & T_{2}: x \leq 0, z \geq 0 . \\
T_{3}: x \leq 0, z \leq 0 . & T_{4}: x \geq 0, z \leq 0 .
\end{array}
$$

We only need to examine the phase portrait of system (31) in the region $T_{1}$ taking into account the symmetries $(x, z, u) \mapsto(-x, z, u),(x, z, u) \mapsto(x,-z,-u)$ and $(x, z, u) \mapsto$ 
$(-x,-z,-u)$. Then system (31) has the same phase portrait on the boundaries of this region as in Section 5.2 except on the non-invariant boundary surface $f_{-}(x, z, u)=0$.

\section{Dynamics in the Interior of the Region $T_{1}$}

In order to examine the orbital behavior inside the region $T_{1}$, we note that the invariant planes $x=0, z=0$ and $u=0$; and the surfaces $h_{0}, h_{2}, h_{3}$ and $f_{-}(x, z, u)=0$ divide the region into ten subregions $T_{11}, T_{12}, \cdots, T_{110}$; see Figures 33 and 34 for more details. The signs of the functions $h_{0}, h_{2}$ and $h_{3}$ in these subregion of $T_{1}$ are shown in Table 12.

The left and right surfaces of the subregion $T_{11}$ in Figure 34 are contained in the surfaces $f_{-}(x, z, u)=0$ and $h_{0}=0$; the left and right planes of the back are contained in the invariant planes $z=0$ and $x=0$, respectively; and the bottom plane is contained in the invariant plane $u=0$, the front surface is contained in the Poincare sphere. Table 13 shows that the orbits in this subregion increase monotonically along the positive directions of the three coordinate axes, indicating that the orbits start at the finite equilibrium point $q_{1}$ or from the adjacent subregion $T_{12}$, and then go to the infinite equilibrium points on the Poincaré sphere.

The left and right surfaces of the subregion $T_{12}$ are contained in the surfaces $h_{0}=0$ and $h_{2}=0$, respectively; the left back plane, right back plane and bottom plane are contained in the invariant planes $z=0, x=0$ and $u=0$, respectively; the top surface and the front surface are contained in the Poincare sphere. The orbits increase monotonically along the positive direction of the three coordinate axes, indicating that the orbits start at the finite equilibrium point $q_{1}$ and finally enter the subregion $T_{11}$ and tend to the infinity equilibrium points on the Poincaré sphere.

The structure of the subregion $T_{13}$ is the same as $Q_{14}$ in Figure 32 except that the left surface in this subregion is completely contained in the surface $h_{2}=0$. In addition the subregions $T_{14}$ and $T_{15}$ have the same structure as the subregions $Q_{15}$ and $Q_{16}$ in Figure 32, respectively. Moreover, the dynamic behavior of the orbits in the subregions $T_{13}, T_{14}$ and $T_{15}$ is the same as that in the subregions $Q_{14}, Q_{15}$ and $Q_{16}$, respectively. That is, the orbits in the subregions $T_{13}$ and $T_{14}$ originate from their respective infinite equilibrium points on the Poincaré sphere, and then the orbits in $T_{13}$ enter the subregion $T_{15}$ and eventually run to the equilibrium points located on the $u$-axis, and the orbits in $T_{14}$ go to the origin $O$ or enter the subregion $T_{13}$.
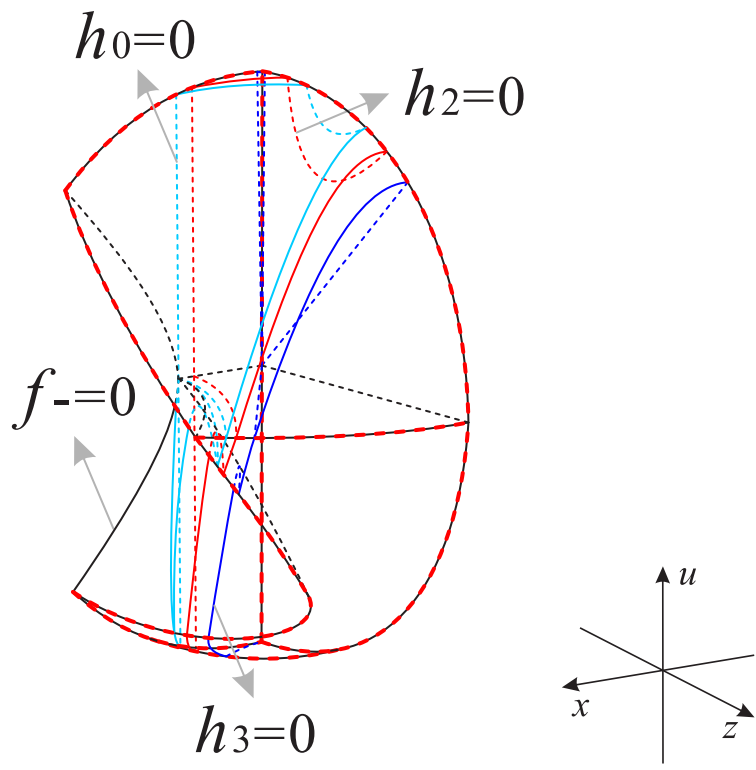

Figure 33. The surfaces $h_{0}, h_{2}$ and $h_{3}$ restricted to the surface $f_{-}(x, z, u)=0$ and the region $T_{1}$ of the Poincaré ball. 


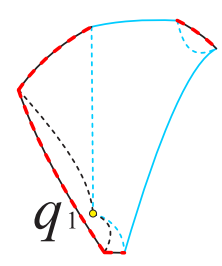

$T_{11}$

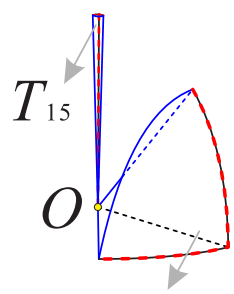

$T_{14}$

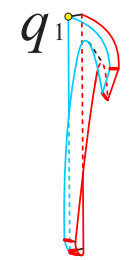

$T_{18}$

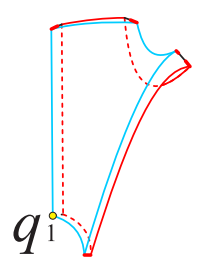

$T_{12}$

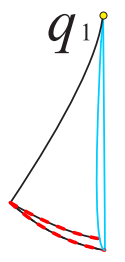

$T_{16}$

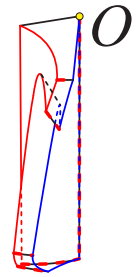

$T_{19}$

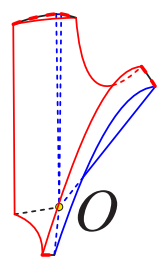

$T_{13}$

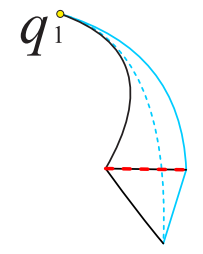

$T_{17}$

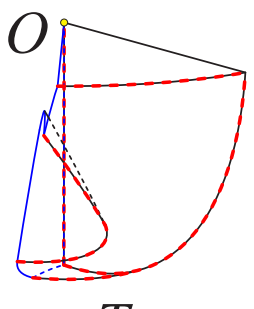

$T_{110}$

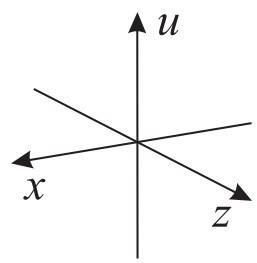

Figure 34. The ten subregions inside the region $T_{1}$ of the Poincaré ball.

Table 12. Signs of functions $h_{0}, h_{2}$ and $h_{3}$ in the subregion of $T_{1}$.

\begin{tabular}{cll}
\hline Functions & Positive & Negative \\
\hline$h_{0}$ & $T_{11}, T_{12}, T_{16}, T_{17}$ & $T_{13}, T_{14}, T_{15}, T_{18}, T_{19}, T_{110}$ \\
\hline$h_{2}$ & $T_{11}, T_{12}, T_{16}, T_{17}, T_{18}$ & $T_{13}, T_{14}, T_{15}, T_{19}, T_{110}$ \\
\hline$h_{3}$ & $T_{11}, T_{12}, T_{13}, T_{16}, T_{17}, T_{18}, T_{19}$ & $T_{14}, T_{15}, T_{110}$ \\
\hline
\end{tabular}

In the subregion $T_{16}$ the front surface is contained in the surface $f_{-}(x, z, u)=0$, the right surface is contained in the surface $h_{0}=0$, the back surface is contained in the invariant plane $z=0$ and the bottom surface is contained in the Poincaré sphere. Table 13 implies that the orbits increase monotonically along the positive direction of the $x$-axis and $z$-axis, and decrease monotonically along the positive direction of the $u$-axis, so that the orbits in this subregion start from the finite equilibrium point $q_{1}$ and finally run to the equilibrium points on the Poincaré shpere on the sphere at infinity, or enter in the outer space through the surface $f_{-}(x, z, u)=0$.

For the subregion $T_{17}$ the left side surface is contained in the surface $f_{-}(x, z, u)=0$, the right side surface is contained in the surface $h_{0}=0$, the top plane is contained in the invariant plane $u=0$ and the front surface is contained in the Poincare sphere. However, it can be followed from Table 13 that the dynamic behavior of the orbits in this subregion is consistent with that in the subregion $T_{16}$. 
In the subregion $T_{18}$ the left side surface and the right side surface are contained in the surfaces $h_{0}=0$ and $h_{2}=0$, respectively; the top plane is contained in the invariant plane $u=0$; and the surfaces on the front part and the bottom part are contained in the Poincare sphere, the middle part of the front surface is contained in the surface $f_{-}(x, z, u)=0$. In this subregion the orbits monotonically decrease along the positive direction of the $x$-axis and $z$-axis, and increase monotonically along the positive direction of the third axis, which means that the orbits start from the finite equilibrium point $q_{1}$ and then enter the subregion $T_{19}$.

The left side surface and the right side surface in the subregion $T_{19}$ are contained in the surfaces $h_{2}=0$ and $h_{3}=0$, respectively; and the composition structure of the remaining part is the same as the corresponding part of the subregion $T_{18}$. The orbits in this subregion monotonically decrease along the positive direction of the three coordinate axes, implying that the orbits start at the infinity equilibrium points on the Poincare sphere or come from the subregion $T_{18}$, and finally tend to the equilibrium points on the $u$-axis.

The front and back parts of the left surface of the subregion $T_{110}$ are contained in the surfaces $h_{3}=0$ and $f_{-}(x, z, u)=0$, respectively; the right and top planes are contained in the invariant planes $x=0$ and $u=0$, respectively; and the front and bottom surfaces are contained in the Poincaré sphere. Note that Table 13 states that the orbits in this subregion decrease monotonically along the positive direction of the $x$-axis and $z$-axis, and increase monotonically along the positive direction of the other coordinate axis. Then the orbits in this subregion start at the infinite equilibrium points on the Poincaré sphere and eventually tend to the finite and infinite equilibrium points on the $u$-axis.

Table 13. Dynamical behavior in the ten subregions.

\begin{tabular}{cll}
\hline Subregions & Corresponding Region & Increase or Decrease \\
\hline$T_{11}$ & $h_{0}>0, h_{2}>0, h_{3}>0, x>0, z>0, u>0$ & $\dot{x}>0, \dot{z}>0, \dot{u}>0$ \\
\hline$T_{12}$ & $h_{0}>0, h_{2}>0, h_{3}>0, x>0, z>0, u>0$ & $\dot{x}>0, \dot{z}>0, \dot{u}>0$ \\
\hline$T_{13}$ & $h_{0}<0, h_{2}<0, h_{3}>0, x>0, z>0, u>0$ & $\dot{x}<0, \dot{z}<0, \dot{u}>0$ \\
\hline$T_{14}$ & $h_{0}<0, h_{2}<0, h_{3}<0, x>0, z>0, u>0$ & $\dot{x}<0, \dot{z}<0, \dot{u}<0$ \\
\hline$T_{15}$ & $h_{0}<0, h_{2}<0, h_{3}<0, x>0, z>0, u>0$ & $\dot{x}<0, \dot{z}<0, \dot{u}<0$ \\
\hline$T_{16}$ & $h_{0}>0, h_{2}>0, h_{3}>0, x>0, z>0, u<0$ & $\dot{x}>0, \dot{z}>0, \dot{u}<0$ \\
\hline$T_{17}$ & $h_{0}>0, h_{2}>0, h_{3}>0, x>0, z>0, u<0$ & $\dot{x}>0, \dot{z}>0, \dot{u}<0$ \\
\hline$T_{18}$ & $h_{0}<0, h_{2}>0, h_{3}>0, x>0, z>0, u<0$ & $\dot{x}<0, \dot{z}>0, \dot{u}<0$ \\
\hline$T_{19}$ & $h_{0}<0, h_{2}<0, h_{3}>0, x>0, z>0, u<0$ & $\dot{x}<0, \dot{z}<0, \dot{u}<0$ \\
\hline$T_{110}$ & $h_{0}<0, h_{2}<0, h_{3}<0, x>0, z>0, u<0$ & $\dot{x}<0, \dot{z}<0, \dot{u}>0$ \\
\hline
\end{tabular}

In short the dynamic behavior of the orbits of system (31) in the region $T_{1}$ can be summarized as follows:

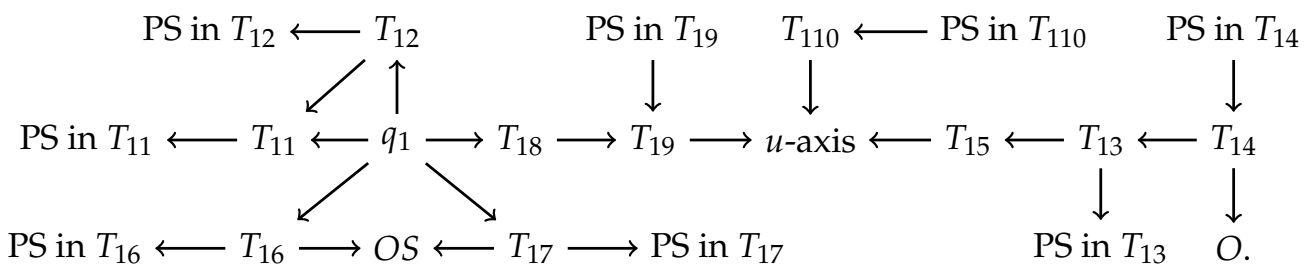

The above flow chart in the region $T_{1}$ indicates that the orbits of system (31) have an $\alpha$-limit at the finite equilibrium point $q_{1}$, and at the infinite equilibrium points located on the Poincaré sphere restricted to the subregions $T_{13}, T_{14}, T_{19}$ and $T_{110}$. Furthermore, the orbits have an $\omega$-limit at the equilibrium points on the $u$-axis; and the equilibrium points at infinity of the Poincaré sphere in the subregions $T_{11}, T_{12}, T_{16}$ and $T_{17}$. 


\section{Conclusions}

By taking advantage of the fact that the cosmological equations (7) remain unchanged under the symmetry $(x, z, u) \mapsto(x,-z,-u)$ when $s \neq 0$, and it remains unchanged under the additional two symmetries $(x, z, u) \mapsto(-x,-z,-u),(x, z, u) \mapsto(-x, z, u)$ when $s=0$ : for a wide range of $s$ in the present paper we completely described the global phase portrait of Hořava-Lifshitz cosmology in the non-flat universe in the case of a non-zero cosmological constant. All of these are located in the physical region of interest $G$ with the invariant boundary surface $f_{+}(x, z, u)=0$ and non-invariant boundary surface $f_{-}(x, z, u)=0$.

For the case $s=0$, the phase portrait of system (31) restricted to the region $G$ shows that the orbits ultimately move to the finite and infinite equilibrium points located on the $u$-axis, or tend to the infinite equilibrium points on the Poincaré sphere in the subregions $Q_{11}, Q_{13}$ and $Q_{17}$ when the universe is closed. Moreover, the orbits of system (31) finally go to the equilibrium points that lie on the $u$-axis or shift to the infinite equilibrium points restricted to subregions $T_{11}, T_{12}, T_{16}$ and $T_{17}$ on the Poincare sphere when the universe is open. Furthermore, we applied the aforementioned symmetries of system (31) to perform simple calculations and found that the unstable equilibrium points $q_{1}$ and $q_{2}$ correspond to the universe being ruled by dark matter.

For the case $s \neq 0$, in addition to the initial conditions on the invariant planes $z=0$ and $u=0$, the phase portrait shows that the orbital evolution of system (7) in region $G$ eventually tends to the equilibrium points at infinity, which are restricted to the subregions $R_{11}, R_{19}, R_{110}$ and $R_{111}, R_{21}$ and $R_{29}$ on the Poincaré sphere when the universe is closed. For an open universe, the orbits of system (7) will go to the infinite equilibrium points restricted to the subregions $S_{11}, S_{19}, S_{21}, S_{22}, S_{27}, S_{29}$ and $S_{210}$ on the Poincare sphere.

For the studied non-flat universe due to some simple calculation combined with the analysis of the phase portrait of system (7) in the previous sections, we conclude from the perspective of cosmology that unstable or non-hyperbolic finite equilibrium points $p_{1}, p_{2}$, $p_{3}, p_{4}$ and $p_{5}$ correspond to the universe being dominated by dark matter. We note that there are two finite equilibrium points, $P_{17}$ and $P_{18}$, that were considered as non-physical points in $[9,10]$, because the value of their corresponding dark matter equation-of-state parameter $\omega_{M}$ was 2 ; but we found that this value is $1 / 3$, and with the notation of our paper corresponds to the equilibrium points $p_{3}$ and $p_{4}$, and of course $1 / 3$ satisfies the physical range $(0,1)$. Furthermore, the previous flow charts show that the orbits of the cosmological model in the region $G$ tend to the equilibrium points at infinity, which can be the late-time state of the universe. Moreover, the finite equilibrium point $p_{5}$ can also be the late-time state of the universe when the initial conditions are on the invariant boundary plane $u=0$. Besides, the orbits will spend a finite lapse of time near the finite equilibrium point $p_{4}$ on the invariant plane $u=0$ before reaching the late-time state $p_{5}$ or the infinite equilibrium points on the Poincaré sphere.

Based on the Hořava-Lifshitz gravity in the non-flat FLRW spacetime with $\Lambda \neq 0$, Equation (5) shows that the Hubble parameter $H$ tends to 0 in the forward time in this cosmological model. This special feature also holds for vanishing curvature (see [16]). For the late-time state $p_{5}$ of the universe on the invariant plane $u=0$, Equations (5) and (6) and $H=\dot{a}(t) / a(t)$ indicate that the Hubble parameter $H$ is an exponential function, and that the scale factor $a(t)$ of the expanding universe is a double exponential function with respect to the time $t$, which expands much more quickly than an usual exponential function.

Author Contributions: Conceptualization, J.L.; Formal analysis, F.G.; software, F.G.; writingoriginal draft, F.G.; writing-review and editing, J.L. All authors have read and agreed to the published version of the manuscript.

Funding: The first author was funded by the National Natural Science Foundation of China (NSFC) through grant Nos. 12172322, 11672259, and the China Scholarship Council (CSC) through grant No. 201908320086. The second author was funded by the Ministerio de Economía, Industria y Competitividad, Agencia Estatal de Investigación grants MTM2016-77278-P (FEDER), the Agència 
de Gestió d'Ajuts Universitaris i de Recerca grant 2017SGR1617, and the H2020 European Research Council grant MSCA-RISE-2017-777911.

Institutional Review Board Statement: Not applicable.

Informed Consent Statement: Not applicable.

Data Availability Statement: Not applicable.

Acknowledgments: We are very grateful to the anonymous reviewers whose comments and suggestions helped improve and clarify this paper.

Conflicts of Interest: The authors declare no conflict of interest.

\section{Appendix A}

We recall the results on normally hyperbolic submanifold according to the monograph of Hirsch et al. [58].

Definition A1. It is assumed that $\phi_{t}$ is a smooth flow on a manifold $M$ and $C$ is a submanifold of $M$, where $C$ is completely composed of the equilibrium points of the flow. If the tangent bundle of $M$ on $C$ is divided into three invariant subbundles $T C, E^{S}, E^{u}$ under $\phi_{t}$ satisfying the conditions

(A1) d $\phi_{t}$ contracts $E^{s}$ exponentially,

(A2) $d \phi_{t}$ expands $E^{u}$ exponentially,

(A3) $\mathrm{TC}=$ tangent bundle of $C$,

then $C$ is called normally hyperbolic submanifold.

For normally hyperbolic submanifolds, one usually has smooth stable and unstable manifolds, and the permanence of these invariant manifolds under small perturbations. To be more precise, we present the following proposition.

Proposition A1. If $C$ is a normally hyperbolic submanifold consisting of equilibrium points for a smooth flow $\phi_{t}$, then there are smooth stable and unstable manifolds, which tangent along $C$ to $E^{S} \oplus T C$ and $E^{u} \oplus T C$, respectively. In addition, the submanifold $C$, and the stable and unstable manifolds, persist under small perturbations of the flow.

\section{References}

1. Hořava, P. Quantum gravity at a Lifshitz point. Phys. Rev. D 2009, 79, 084008. [CrossRef]

2. Antoniadis, I.; Cotsakis, S. Geodesic incompleteness and partially covariant gravity. Universe 2021, 7, 126. [CrossRef]

3. Bajardi, F.; Bascone, F.; Capozziello, S. Renormalizability of alternative theories of gravity: Differences between power counting and entropy argument. Universe 2021, 7, 148. [CrossRef]

4. Abreu, E.M.; Mendes, A.C.; Oliveira-Neto, G.; Neto, J.A.; Rodrigues, L.R.; de Oliveira, M.S. Hořava-Lifshitz cosmological models with noncommutative phase space variables. Gen. Relativ. Gravit. 2019, 51, 95. [CrossRef]

5. Brandenberger, R. Matter bounce in Hořava-Lifshitz cosmology. Phys. Rev. D 2009, 80, 043516. [CrossRef]

6. Kobayashi, T.; Urakawa, Y.; Yamaguchi, M. Large scale evolution of the curvature perturbation in Hořava-Lifshitz cosmology. J. Cosmol. Astropart. Phys. 2009, 2009, 015. [CrossRef]

7. Carloni, S.; Elizalde, E.; Silva, P.J. An analysis of the phase space of Hořava-Lifshitz cosmologies. Class. Quantum Gravity 2010, 27, 045004. [CrossRef]

8. Chen, B. On Hořava-Lifshitz cosmology. Chin. Phys. C 2011, 35, 429-435. [CrossRef]

9. Leon, G.; Saridakis, E.N. Phase-space analysis of Hořava-Lifshitz cosmology. J. Cosmol. Astropart. Phys. 2009, 2009, 006. [CrossRef]

10. Leon, G.; Fadragas, C.R. Cosmological Dynamical Systems: And Their Applications; Lambert Academic Publishing, GmbH \& Co. KG: Saarbrücken, Germany, 2012.

11. Leon, G.; Paliathanasis, A. Extended phase-space analysis of the Hořava-Lifshitz cosmology. Eur. Phys. J. C 2019, 79, 746. [CrossRef]

12. Paliathanasis, A.; Leon, G. Cosmological solutions in Hořava-Lifshitz scalar field theory. Zeitschrift für Naturforschung A 2020, 75, 523-532. [CrossRef]

13. Fadragas, C.R.; Leon, G.; Saridakis, E.N. Dynamical analysis of anisotropic scalar-field cosmologies for a wide range of potentials. Class. Quantum Gravity 2014, 31, 075018. [CrossRef]

14. Gao, F.B.; Llibre, J. Global dynamics of the Hořava-Lifshitz cosmological system. Gen. Relativ. Gravit. 2019, 51, 152. [CrossRef] 
15. Gao, F.B.; Llibre, J. Global dynamics of Hořava-Lifshitz cosmology with non-zero curvature and a wide range of potentials. Eur. Phys. J. C 2020, 80, 137. [CrossRef]

16. Gao, F.B.; Llibre, J. Global dynamics of the Hořava-Lifshitz cosmology in the presence of non-zero cosmological constant in a flat space. arXiv 2021, arXiv:2111.08377v1. Available online: https:/ / arxiv.org/abs/2111.08377v1 (accessed on 16 November 2021).

17. Gao, X.; Wang, Y.; Brandenberger, R.; Riotto, A. Cosmological perturbations in Hořava-Lifshitz gravity. Phys. Rev. D 2010, 81, 083508. [CrossRef]

18. Escobar, D.; Fadragas, C.R.; Leon, G.; Leyva, Y. Asymptotic behavior of a scalar field with an arbitrary potential trapped on a Randall-Sundrum's braneworld: The effect of a negative dark radiation term on a Bianchi I brane. Astrophys. Space Sci. 2014, 349, 575-602. [CrossRef]

19. Alho, A.; Hell, J.; Uggla, C. Global dynamics and asymptotics for monomial scalar field potentials and perfect fluids. Class. Quantum Gravity 2015, 32, 145005. [CrossRef]

20. Kim, H.C. Inflation as an attractor in scalar cosmology. Mod. Phys. Lett. A 2013, 28, 1350089. [CrossRef]

21. Chervon, S.; Fomin, I.; Yurov, V.; Yurov, A. Scalar Field Cosmology; World Scientific Publishing Co. Pte. Ltd.: Singapore, 2019.

22. Kiritsis, E.; Kofinas, G. Hořava-Lifshitz cosmology. Nucl. Phys. B 2009, 821, 467-480. [CrossRef]

23. Lepe, S.; Saavedra, J. On Hořava-Lifshitz cosmology. Astrophys. Space Sci. 2014, 350, 839-843. [CrossRef]

24. Cordero, R.; García-Compeán, H.; Turrubiates, F.J. A phase space description of the FLRW quantum cosmology in Hořava-Lifshitz type gravity. Gen. Relativ. Gravit. 2019, 51, 138. [CrossRef]

25. Nilsson, N.A.; Czuchry, E. Hořava-Lifshitz cosmology in light of new data. Phys. Dark Universe 2019, 23, 100253. [CrossRef]

26. Saridakis, E.N. Aspects of Hořava-Lifshitz cosmology. Int. J. Mod. Phys. D 2011, 20, 1485-1504. [CrossRef]

27. Tawfik, A.; Dahab, E.A.E. FLRW cosmology with Hořava-Lifshitz gravity: Impacts of equations of state. Int. J. Theor. Phys. 2017, 56, 2122-2139. [CrossRef]

28. Cognola, G.; Myrzakulov, R.; Sebastiani, L.; Vagnozzi, S.; Zerbini, S. Covariant Hořava-like and mimetic Horndeski gravity: Cosmological solutions and perturbations. Class. Quantum Gravity 2016, 33, 225014. [CrossRef]

29. Tavakoli, F.; Vakili, B.; Ardehali, H. Hořava-Lifshitz scalar field cosmology: Classical and quantum viewpoints. Adv. High Energy Phys. 2021, 2021, 6617910. [CrossRef]

30. Casalino, A.; Rinaldi, M.; Sebastiani, L.; Vagnozzi, S. Alive and well: Mimetic gravity and a higher-order extension in light of GW170817. Class. Quantum Gravity 2019, 36, 017001. [CrossRef]

31. Saridakis, E.N. Hořava-Lifshitz dark energy. Eur. Phys. J. C 2010, 67, 229-235. [CrossRef]

32. Jamil, M.; Saridakis, E.N. New agegraphic dark energy in Hořava-Lifshitz cosmology. J. Cosmol. Astropart. Phys. 2010, 2010, 028. [CrossRef]

33. Setare, M.R.; Jamil, M. Holographic dark energy with varying gravitational constant in Hořava-Lifshitz cosmology. J. Cosmol. Astropart. Phys. 2010, 2010, 010. [CrossRef]

34. Setare, M.R.; Momeni, D.; Moayedi, S.K. Interacting dark energy in Hořava-Lifshitz cosmology. Astrophys. Space Sci. 2011, 338, 405-410. [CrossRef]

35. Sheykhi, A.; Ghaffari, S.; Moradpour, H. Ghost dark energy in the deformed Hořava-Lifshitz cosmology. Int. J. Mod. Phys. D 2019, 28, 1950080. [CrossRef]

36. Cai, R.G.; Cao, L.M.; Ohta, N. Topological black holes in Hořava-Lifshitz gravity. Phys. Rev. D 2009, 80, 024003. [CrossRef]

37. Bhattacharjee, M. Gravitational radiation and black hole formation from gravitational collapse in theories of gravity with broken Lorentz symmetry. Ph.D. Thesis, Baylor University, Waco, TX, USA, 2019; p. 22585106.

38. Pourhassan, B. PV criticality of the second order quantum corrected Hořava-Lifshitz black hole. Eur. Phys. J. C 2019, 79, 740. [CrossRef]

39. Du, Y.Z.; Zhao, H.H.; Zhang, L.C. Phase transition of the Horava-Lifshitz AdS black holes. Int. J. Theor. Phys. 2021, 60, $1963-1971$. [CrossRef]

40. Poshteh, M.B.J.; Mann, R.B. Thermodynamics of $z=4$ Hořava-Lifshitz black holes. Phys. Rev. D 2021, 103, 104024. [CrossRef]

41. Mestra-Páez, J.; Peña, J.M.; Restuccia, A. Gravitational waves in Hořava-Lifshitz anisotropic gravity. arXiv 2021, arXiv:2105.00951v2.

42. Lin, K.; Yang, N.; Li, J. Electromagnetic quasinormal modes in Hořava-Lifshitz gravity. Int. J. Theor. Phys. 2011, 50, 48-55. [CrossRef]

43. Borzou, A.; Lin, K.; Wang, A. Static electromagnetic fields and charged black holes in general covariant theory of Hořava-Lifshitz gravity. J. Cosmol. Astropart. Phys. 2012, 2012, 025. [CrossRef]

44. Bellorín, J.; Restuccia, A.; Tello-Orti, F. Anisotropic coupling of gravity and electromagnetism in Hořava-Lifshitz theory. Phys. Rev. D 2009, 98, 104018. [CrossRef]

45. Restucciaa, A.; Tello-Ortizb, F. Pure electromagnetic-gravitational interaction in Hořava-Lifshitz theory at the kinetic conformal point. Eur. Phys. J. C 2020, 80, 86. [CrossRef]

46. Wang, A.Z. Hořava gravity at a Lifshitz point: A progress report. Int. J. Mod. Phys. D 2017, 26, 1730014. [CrossRef]

47. Mukohyama, S. Hořava-Lifshitz cosmology: A review. Class. Quantum Gravity 2010, 27, 223101. [CrossRef]

48. Sotiriou, T.P. Hořava-Lifshitz gravity: A status report. J. Phys. Conf. Ser. 2011, 283, 012034. [CrossRef]

49. Appignani, C.; Casadio, R.; Shankaranarayanan, S. The cosmological constant and Hořava-Lifshitz gravity. J. Cosmol. Astropart. Phys. 2010, 2010, 006. [CrossRef] 
50. Akarsu, Ö.; Barrow, J.D.; Escamilla, L.A.; Vazquez, J.A. Graduated dark energy: Observational hints of a spontaneous sign switch in the cosmological constant. Phys. Rev. D 2020, 101, 063528. [CrossRef]

51. Carlip, S. Hiding the cosmological constant. Phys. Rev. Lett. 2019, 123, 131302. [CrossRef]

52. Handley, W. Curvature tension: Evidence for a closed universe. Phys. Rev. D 2021, 103, L041301. [CrossRef]

53. Valentino, E.D.; Melchiorri, A.; Silk, J. Planck evidence for a closed universe and a possible crisis for cosmology. Nat. Astron. 2020, 4, 196-203. [CrossRef]

54. Dumortier, F.; Llibre, J.; Artés, J.C. Qualitative Theory of Planar Differential Systems; Springer: Berlin/Heidelberg, Germany, 2006.

55. Cima, A.; Llibre, J. Bounded polynomial vector fields. Trans. Am. Math. Soc. 1990, 318, 557-579. [CrossRef]

56. Kuznetsov, Y.A. Elements of Applied Bifurcation Theory; Springer: New York, NY, USA, 2004.

57. Álvarez, M.J.; Ferragut, A.; Jarque, X. A survey on the blow up technique. Int. J. Bifurc. Chaos 2011, 21, 3103-3118. [CrossRef]

58. Hirsch, M.W.; Pugh, C.C.; Shub, M. Invariant Manifolds; Springer: Berlin/Heidelberg, Germany, 1977. 